\title{
Electrothermal Analysis of Gallium Nitride Island Transistor eHEMT Devices for Fault Tolerant Design
}

by

\author{
Evan Christopher de Sousa, B.Eng.
}

A thesis submitted to the Faculty of Graduate and Postdoctoral Affairs in partial fulfillment of the requirements for the degree of

\section{Master of Applied Science}

in

\section{Electrical and Computer Engineering}

\author{
Ottawa-Carleton Institute for Electrical and Computer Engineering \\ Department of Electronics \\ Carleton University \\ Ottawa, Ontario, Canada
}

May 2018

Copyright (C) 2018

Evan Christopher de Sousa 


\section{Abstract}

A full 3D thermal simulation structure was built using Atar based on the GS66516T device from GaN Systems with individually controllable generation regions for each island transistor in an array. The thermal model was linked to electrical SPICE models in OptiSPICE to produce a combined electrothermal simulator to be used to investigate the device behaviour when cells in the array are deactivated. The individual

electrical, thermal, and electrothermal simulations were all verified against models, previous simulations, and physical device testing to ensure that the simulations behaved accordingly. The results of the electrothermal simulation verification showed that there was a less than $5 \%$ error between the measured and simulated values, providing an accurate simulation of the electrothermal behaviour of the GS66516T device. Once the simulations were verified, electrothermal simulations were run for the case of one, two, three, and four random cells deactivated. From the results of these simulations, data was gathered and interpreted to show the possibilities of the electrothermal simulations for determining device behaviour with cells deactivated. 


\section{Acknowledgements}

In no particular order, I would like to thank the following people for all they contributed to the completion of this thesis:

My supervisor, Professor Steven McGarry, who helped guide the entire project to completion though his wisdom and sage advice. Thanks for taking all the setbacks we encountered in stride, no problem felt insurmountable thanks to you.

Professor Tom Smy, although not officially my supervisor, was always there whenever I needed his help and was instrumental in getting the software to operate correctly and tailoring functionality to the requirements of this project. Thanks for always having an idea what to do (and a sense of humour) whenever I came to you with a software issue, debugging was never a frustrating exercise thanks to you.

My fellow graduate students in our research group, thanks for helping solve problems and coming up with ideas during our weekly meetings. No matter who left and who joined, the group never stopped being a valuable source of advice and inspiration.

The Department of Electronics staff: Blazenka Power, Anna Lee, and Sylvie Beekmans, for keeping the department running like a well-oiled machine. Scott Bruce, for

providing the computing resources and help I needed to get my simulations running on the servers. Steve MacLaurin, for providing me equipment I needed for getting my computer for controlling my physical testing set up.

GaN Systems, especially Cameron McKnight-MacNeil, for providing me with the information I needed for my simulations and for the example devices for testing. 
OCE, NSERC, and GaN Systems for jointly funding my research for this thesis.

Rob Vandusen, for providing me with the tools I needed to prepare my physical devices and working with me to figure out how to connect them when they were polished.

Hoskin Scientific and FLIR Systems, in particular Jack Vincent and Manny Alsaid, for agreeing to lend me the thermal camera that was key to proving the validity of my simulations.

Family and friends, thanks for all your support even though you had no idea what I was talking about most of the time.

Finally, none of this would have been possible if it wasn't for the help, guidance, advice, and support of Samantha Trifoli. Thanks for keeping me going all those times I wanted to give up and helping me out as much as humanly possible. I can honestly say that I couldn't have done this without you. 


\section{Table of Contents}

Abstract $\quad$ ii

Acknowledgments

iii

Table of Contents $\quad$ v

List of Figures viii

List of Tables $\quad$ xiii

List of Terms $\quad$ xiv

List of Acronyms $\quad$ XV

List of Symbols $\quad$ xvii

1 Introduction 1

1.1 Motivation . . . . . . . . . . . . . . . . 1

1.2 Thesis Objective . . . . . . . . . . . . . . . . . . 3

1.3 Thesis Outline....................... 4

2 Background 5

2.1 Semiconductors . . . . . . . . . . . . . . . . 5

2.1.1 Semiconductor Fundamentals ............. 5 
2.1.2 High Electron Mobility Transistors . . . . . . . . . . . . . . . 11

2.2 Thermodynamics . . . . . . . . . . . . . . . . . . 19

2.2.1 Heat Transfer Methods . . . . . . . . . . . . . . . . . . . 19

2.2.2 Fourier's Law and the Heat Equation . . . . . . . . . . . . . . 20

2.2.3 Boundary Conditions . . . . . . . . . . . . . . . . . . . 22

2.3 Thermal Effects in $\mathrm{GaN} \ldots \ldots . \ldots . . \ldots 23$

2.3.1 Recoverable Effects . . . . . . . . . . . . . . . . . . 24

2.3.2 Permanent Effects . . . . . . . . . . . . . . . 25

2.4 Electrical and Thermal Simulation . . . . . . . . . . . . 26

2.4.1 SPICE Basics . . . . . . . . . . . . . 26

2.4.2 Finite Difference Method . . . . . . . . . . . . . . . . . 29

2.4 .3 Rule of Mixtures . . . . . . . . . . . . . . . . . . . . . 31

2.4.4 Simulators .................... 33

3 Experiment Setup and Simulation Correlation 37

3.1 Simulation Setup . . . . . . . . . . . . . . . . . . 37

3.1.1 Simulator Selection . . . . . . . . . . . . . . 37

3.1.2 OptiSPICE Device from GaN Systems LTSpice Netlist . . . . 38

3.1.3 Device Structure Setup for Atar . . . . . . . . . . . . . . . . . 41

3.1.4 Electrothermal Setup for OptiSPICE . . . . . . . . . . . 50

3.2 Simulator Correlation . . . . . . . . . . . . . . . . . 51

3.2.1 OptiSPICE Electrical Simulation Correlation . . . . . . . . . . 51

3.2.2 Atar Thermal Simulation Correlation . . . . . . . . . . . . 54

3.2.3 OptiSPICE Electrothermal Simulation Verification . . . . . 54

4 Electrothermal Simulation Results $\quad 78$

4.1 Electrothermal Simulation Setup . . . . . . . . . . . . . 78

4.2 Location of Highest Temperature Cell . . . . . . . . . . . . . . . . . . 79 
$4.3 \Delta \mathrm{T}$ of Highest and Lowest Temperature on Chip . . . . . . . . . . . . 80

4.4 Maximum Cell Temperature Versus Number of Deactivated Cells . 80

4.5 Extreme Cases for Four Deactivated Cells . . . . . . . . . . . . . 85

$\begin{array}{llr}5 & \text { Conclusions } & 87\end{array}$

5.1 Summary ............................. 87

5.2 Thesis Contributions . . . . . . . . . . . . . . . 87

5.3 Future Work . . . . . . . . . . . . . . . . . . . 88

$\begin{array}{lr}\text { References } & 90\end{array}$

$\begin{array}{lll}\text { Appendix A Model Files } & 95\end{array}$

A.1 GS66516T LTSpice Model . . . . . . . . . . . . . . . . . 95

$\begin{array}{lll}\text { Appendix B Simulation Input Files } & 98\end{array}$

B.1 OptiSPICE Electrothermal Simulation Input Example Code . . . . . 98

B.2 OptiSPICE Electrical Simulation Input Code . . . . . . . . . . . . . 105

$\begin{array}{lll}\text { Appendix C Simulation Output Files } & 107\end{array}$

C.1 Atar Thermal Simulation Comparison Output . . . . . . . . . . . . . 107

C.2 OptiSPICE Electrothermal Simulation Comparison Output . . . . . . 110 


\section{List of Figures}

1.1 Radar chart showing the key characteristics of GaN relative to those of Si and GaAs (adapted from [4]). . . . . . . . . . . 2

1.2 Die Yield vs. Device Size for GaN (adapted from [6]) . . . . . . . . 3

2.1 Basic energy band diagram. . . . . . . . . . . . . . . . . 7

2.2 Energy band diagram showing the Fermi level position in doped semiconductors. . . . . . . . . . . . . . . . . . 8

2.3 Energy band diagrams of a $p$ - $n$ junction at equilibrium and at forward bias. . . . . . . . . . . . . . . . . . . . 10

2.4 Cross-section and energy band diagrams showing band bending in metal-oxide-semiconductors. . . . . . . . . . . . . . . . . . . . . . . . 12

2.5 Energy band diagrams showing the three different types of heterojunction interfaces: straddling gap, staggered gap, and broken gap. . . . . 14

2.6 Energy band diagram of a straddling gap heterojunction showing band bending. . . . . . . . . . . . . . . . . .

2.7 Energy band diagram of a heterojunction showing the formation of the

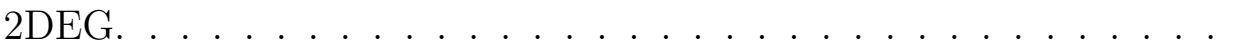

2.8 Location and direction of polarization and electric fields in GaN and strained AlGaN. . . . . . . . . . . . . . . . . 
2.9 The movement of electrons from the surface donor states and the formation of the 2DEG at the heterointerface in an AlGaN/GaN heterostructure (adapted from $[18]) . \ldots \ldots \ldots$

2.10 Cross-section diagram of a typical GaN eHEMT structure. . . . . . . 19

2.11 AlGaN/GaN DC characteristics with and without self-heating effects (used with permission from $[23]) . \ldots \ldots \ldots \ldots \ldots$

2.12 A comparison of some SPICE statements, their representative circuit elements, and stamps used to map the netlist into a matrix equation

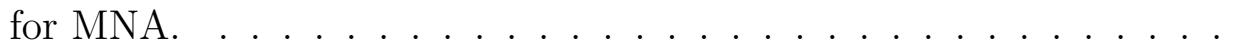

2.13 Example SPICE circuit used for demonstrating MNA matrix equation derivation. . . . . . . . . . . . . . . . . . 28

2.14 Two dimensional finite difference mesh example. . . . . . . . . . . 30

2.15 Visual representation of the fiber, matrix, volume fractions, and transverse/longitudinal directions for the rule of mixtures. . . . . . . . .

2.16 An example structure showing an array of high thermal conductivity via surrounded by low thermal conductivity oxide. . . . . . . . . . . 33

2.17 Quad tree mesh example for (a) 2D mesh and (b) 3D mesh (used with permission from $[35]) \ldots \ldots \ldots \ldots \ldots \ldots$

3.1 3D CAD representation of the chip structure to be simulated. . . . . 39

3.2 SPICE circuit built from GS66516T model netlist (example netlist shown in Appendix A.1) . . . . . . . . . . . . . . . .

3.3 3D CAD model of the thermal correlation structure and Atar output of the same structure. (Note: The visual glitches seen in Figure 3.3b are due to the coinciding generation region and GaN surfaces) . . .

3.4 Layers of the single cell stack-up built in Atar. (Note: The visual glitch seen in Figure 3.4a/3.4b is due to the coinciding generation region and GaN surfaces $) \ldots \ldots \ldots \ldots \ldots \ldots \ldots$ 
3.5 A mirrored cell structure showing a pair of cells that share a source

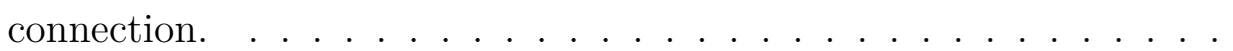

3.6 The full chip structure up to the M2 layer showing the 36 individual

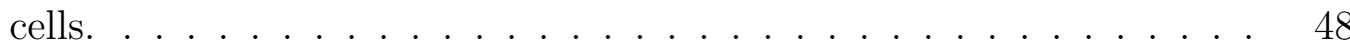

3.7 Number map of the 36 cells in the full chip structure used for reference

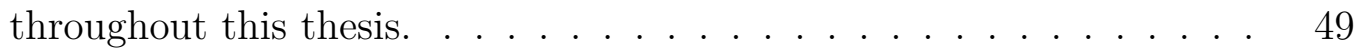

3.8 OptiSPICE electrothermal SPICE circuit of common sources (left), and of an individual cell (right, red box) . . . . . . . . . . . 52

$3.9 \mathrm{I}_{\mathrm{DS}}$ vs $\mathrm{V}_{\mathrm{DS}}$ curves comparing the electrical outputs of the GS66516T datasheet, the LTSpice simulation using the GaN Systems' full device model, and the OptiSPICE simulation using island transistors in a

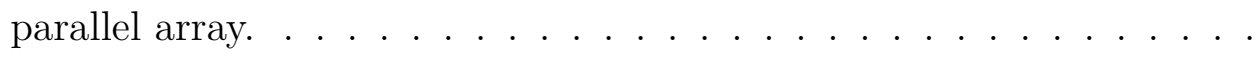

3.10 Atar thermal simulation results for comparison to the GaN Systems results shown in Figure $3.11 \ldots \ldots \ldots \ldots \ldots$

3.11 GaN Systems commercial thermal simulation results for comparison to Atar results as shown in Figure $3.10 \ldots \ldots \ldots$

3.12 KEPCO RA55 rack mount with MST power supplies used for the high voltage/high current powering of the GaN devices. . . . . . . . . 57

3.13 KEPCO RA55 original power supply connectors, where connections were made with bare 14AWG solid core wires. . . . . . . . . . 58

3.14 3D CAD model of the connector designed for high voltage testing with banana plug inputs for the KEPCO RA55 . . . . . . . . . . 59

3.15 The completed KEPCO RA55 power supply connector based on the design in Figure $3.14 \ldots \ldots \ldots$. . . . . . . . . . . 60

3.16 KEPCO RA55 power supply unit with the new connectors for high voltage testing with banana plug inputs. . . . . . . . . . . . . 61 
3.17 Temptronic TPO315B temperature controller used for cooling the chuck

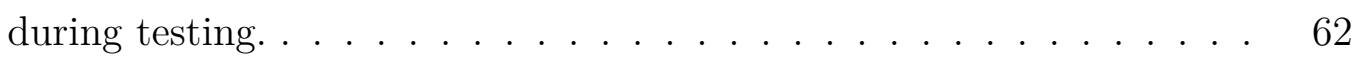

3.18 Karl Suss probe station with temperature controlled chuck. . . . . . . 62

3.192 " $\times 2$ " $\times 1 / 4$ " aluminium heat sink block used to dissipate heat away from the device during testing. . . . . . . . . . . . . . 63

3.20 Buehler ECOMET III polisher/grinder used for mechanically polishing the upper layers of the device down to the copper RDL on the chip

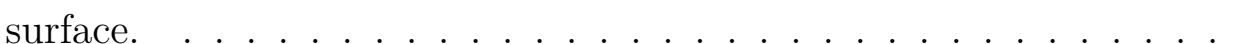

3.21 Images of a device after mechanical polishing showing the copper RDL and identifying connections. . . . . . . . . . . . . . 65

3.22 A failed unpolished device after testing which resulted in a melted package due to overheating. . . . . . . . . . . . 66

3.23 Aluminium block temperature and chip power dissipation at steadystate over time. . . . . . . . . . . . . . . . . . 6 68

3.24 Thermal image of an unpolished device dissipating $30 \mathrm{~W}$ of power (legend in units of $\left.{ }^{\circ} \mathrm{C}\right) \ldots \ldots \ldots \ldots \ldots \ldots \ldots \ldots$

3.25 A graphical representation of the setup used for the thermal analysis. 69

3.26 The thermal analysis representing the physical testing of the device. . 70

3.27 A polished device mounted to the heat sink block showing connections made for testing. . . . . . . . . . . . . . . . . . 72

3.28 Infrared imaging results of a polished test device shown with two different temperature scales (legend in units of ${ }^{\circ} \mathrm{C}$ ). . . . . . . 74

3.29 Infrared imaging test of polyimide (Kapton) using a powered resistor to illustrate the effect of polyimide on the thermal imaging of the polished device $\left(\right.$ legend in units of $\left.{ }^{\circ} \mathrm{C}\right) \ldots \ldots \ldots \ldots \ldots \ldots \ldots$

3.30 Approximate thermal comparison of the Atar output and the thermal

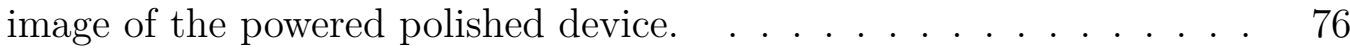


3.31 Thermal image of the unpolished device and the extraction of the max-

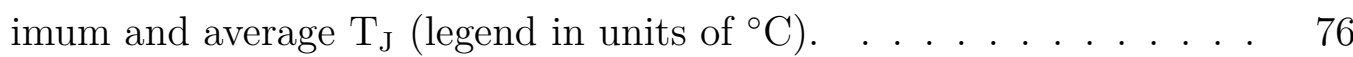

4.1 The maximum temperature cell location probability distribution for an increasing number of deactivated cells. . . . . . . . . . . . . . 81

4.2 Reference cell number and colour scheme for the probability distribu-

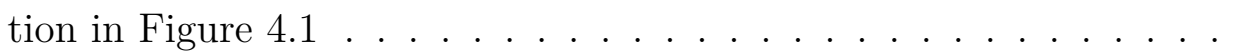

4.3 Depiction of the highest temperature cell movement with an increasing number of deactivated cells. . . . . . . . . . . . . . . . . 82

4.4 Temperature gradient distributions for an increasing amount of deactivated cells relative to the lowest temperature inactive cell. Units for the bins (x-axis) are in ${ }^{\circ} \mathrm{C} \ldots \ldots \ldots \ldots$

4.5 Temperature gradient distributions for an increasing amount of deactivated cells relative to the lowest temperature active cell. Units for

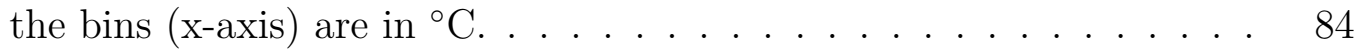

4.6 Depiction of the maximum cell temperature increasing and range spreading as the number of deactivated cells increases. Units for the maxi-

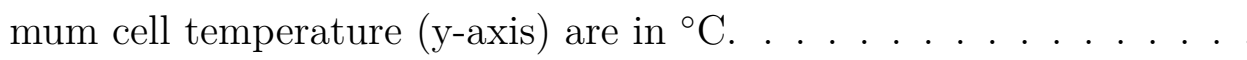




\section{List of Tables}

1.1 Comparison of various semiconductor properties of Si, GaAs, and GaN (adapted from [5]). . . . . . . . . . . . . . . . 2

3.1 List of derivatives for arbitrary current sources and voltage-dependent capacitors. . . . . . . . . . . . . . . . . . 41

3.2 Summary of the Atar single cell full structure layout (without substrate). 45

3.3 Summary of OptiSPICE electrothermal netlist elements . . . . . . . 51

3.4 Results of thermal analysis based on physical test data. . . . . . . . . 71

3.5 Comparison between measurements and simulation for electrothermal behaviour. . . . . . . . . . . . . . . . . 77

4.1 Number of unique combinations for each case considered and number of tests run. . . . . . . . . . . . . . . . . . . . 79

4.2 Results for several extreme cases of four deactivated cells. . . . . . . . 86 


\section{List of Terms}

cell

One single island transistor, part of the transistor array that makes a full chip.

chip

Array of island transistors connected in parallel to form one logical transistor with equivalent gate width $\left(W_{g}\right)$ of the sum of all $W_{g}$ of connected islands.

\section{device}

The full device consists of the chip embedded in the GaNPX package as detailed in $[1]$. 


\section{List of Acronyms}

2DEG two dimensional electron gas.

AlGaN aluminium gallium nitride.

CAD computer-aided design.

CPU central processing unit.

GaN gallium nitride.

HEMT high electron mobility transistor.

IGBT insulated-gate bipolar transistor.

MDMR multi-dimensional model reduction.

MNA modified nodal analysis.

MOSFET metal-oxide-semiconductor field-effect transistor.

NDA non-disclosure agreement.

PCB printed circuit board.

RDL redistribution layer.

$\mathbf{R F}$ radio frequency. 
SPICE Simulation Program with Integrated Circuit Emphasis.

TIM thermal interface material. 


\section{List of Symbols}

$E_{F} \quad$ Fermi level $(\mathrm{eV})$

$E_{c} \quad$ Bottom of the conduction band $(\mathrm{eV})$

$E_{v} \quad$ Top of the valence band $(\mathrm{eV})$

$E_{i} \quad$ Intrinsic Fermi level (eV)

$k \quad$ Boltzmann constant $(\mathrm{J} / \mathrm{K}$ or $\mathrm{eV} / \mathrm{K})$

$T$ Temperature $\left(\mathrm{K}\right.$ or $\left.{ }^{\circ} \mathrm{C}\right)$

$q \quad$ Electron charge $(\mathrm{C})$

$n \quad$ Concentration of electrons in the conduction band $\left(\mathrm{cm}^{-3}\right)$

$p \quad$ Concentration of holes in the valence band $\left(\mathrm{cm}^{-3}\right)$

$N_{c} \quad$ Effective density of conduction band states $\left(\mathrm{cm}^{-3}\right)$

$N_{v} \quad$ Effective density of valence band states $\left(\mathrm{cm}^{-3}\right)$

$\phi_{B} \quad$ Bulk potential (V)

$E_{S} \quad$ Donor state level $(\mathrm{eV})$

$n_{s} \quad$ 2DEG electron density $\left(\mathrm{cm}^{-2}\right)$

$\kappa \quad$ Thermal conductivity $(\mathrm{W} / \mathrm{mK})$

$h \quad$ Heat transfer coefficient $\left(\mathrm{W} / \mathrm{m}^{2} \mathrm{~K}\right)$

$\epsilon \quad$ Surface emissivity

$\sigma \quad$ Stefan-Boltzmann constant $\left(\mathrm{W} / \mathrm{m}^{2} \mathrm{~K}^{4}\right)$

$q_{n} \quad$ Heat transfer rate/Heat flux $\left(\mathrm{W} / \mathrm{m}^{2}\right)$

$R_{\theta} \quad$ Thermal resistance in $\left(\mathrm{K} / \mathrm{W}\right.$ or $\left.{ }^{\circ} \mathrm{C} / \mathrm{W}\right)$

g Volumetric thermal energy generation $\left(\mathrm{W} / \mathrm{m}^{3}\right)$

$\rho \quad$ Material density $\left(\mathrm{kg} / \mathrm{m}^{3}\right)$

$c_{p} \quad$ Specific heat of a material $(\mathrm{J} / \mathrm{kg} \cdot \mathrm{K})$ 


\section{Chapter 1}

\section{Introduction}

\subsection{Motivation}

Gallium nitride (GaN) high electron mobility transistor (HEMT) devices first appeared commercially around 2004 although research into GaN HEMTs has been ongoing since the early 90's. This technology been gaining popularity in the radio frequency $(\mathrm{RF})$ and power conversion market as research and development leads to new capabilities, increased usability and decreased manufacturing cost [2]. GaN is emerging as the leading technology to replace silicon power MOSFETs and insulatedgate bipolar transistors (IGBTs) and even silicon carbide-based transistors in power applications [3] and is already seeing use in the areas of consumer electronics, renewable energy, industrial applications, and the automotive industry.

GaN technology has many benefits over other semiconducting materials as outlined in Figure 1.1. GaN has a wider bandgap than both silicon and gallium arsenide (GaAs) which provides a higher breakdown field and allows for GaN devices to operate at much higher voltages. The wide bandgap also allows for operation at higher temperatures as the energy required to thermally excite an electron to the conduction band is greater than materials with a smaller bandgap. As can be seen from 
the values given in Table 1.1, GaN also benefits from a high electron mobility and saturation velocity which leads to high current density. All these factors make GaN well suited to the future of power and RF applications. The advancement of GaN

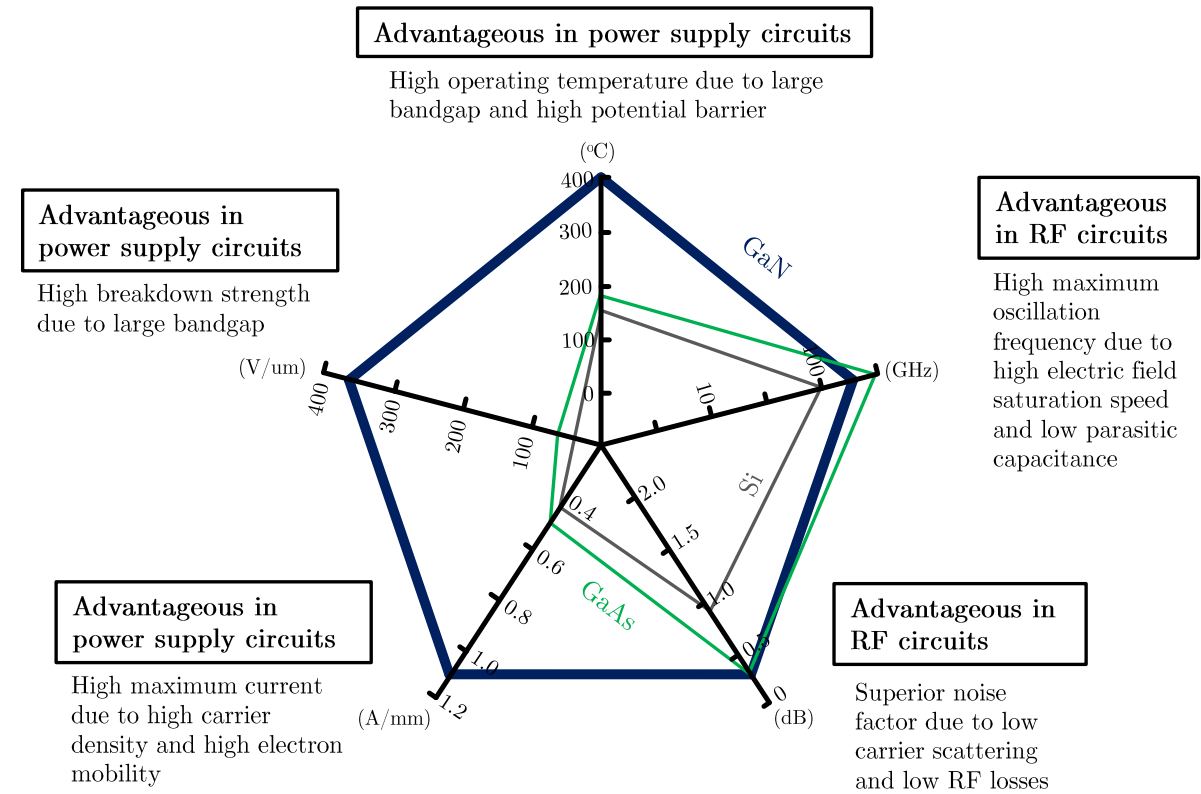

Figure 1.1: Radar chart showing the key characteristics of GaN relative to those of Si and GaAs (adapted from [4]).

Table 1.1: Comparison of various semiconductor properties of $\mathrm{Si}, \mathrm{GaAs}$, and $\mathrm{GaN}$ (adapted from [5]).

\begin{tabular}{|l||c|c|c|}
\hline & Si & GaAs & GaN \\
\hline Bandgap $(\mathrm{eV})$ & 1.1 & 1.42 & 3.4 \\
\hline Electron Mobility $\left(\mathrm{cm}^{2} / \mathrm{Vs}\right)$ & 1350 & 8500 & $\sim 2000$ \\
\hline Saturation Velocity $\left(10^{7} \mathrm{~cm} / \mathrm{s}\right)$ & 1.0 & 1.0 & 2.5 \\
\hline Critical Breakdown Field $(\mathrm{MV} / \mathrm{cm})$ & 0.3 & 0.4 & 3.3 \\
\hline Thermal Conductivity $(\mathrm{W} / \mathrm{cmK})$ & 1.5 & 0.43 & 1.3 \\
\hline
\end{tabular}

technology has not been without setbacks as the expense of GaN wafers and GaN grown on silicon carbide and sapphire has held back widespread adoption. Silicon has more recently been used as a substrate on which to grow GaN due to its inexpensive nature and well-developed infrastructure however silicon is not an ideal base for GaN due to the lattice mismatch and the mismatch of thermal expansion coefficients 
[2]. This can lead to a high defect density and low yield as device size increases as shown in Figure 1.2. Finding ways to increase yield through fault tolerant design is an important area for research and forms the basis for the motivation of this thesis.

Die Yield vs. Device Size

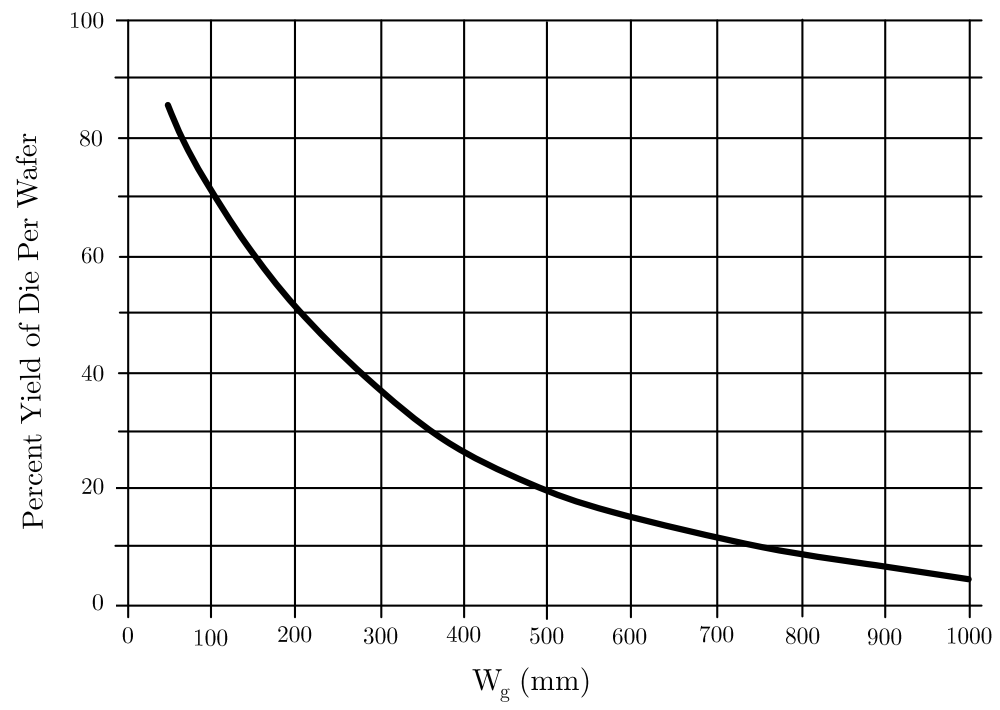

Figure 1.2: Die Yield vs. Device Size for GaN (adapted from [6])

\subsection{Thesis Objective}

The goal of this thesis is to examine the combined electrical and thermal behaviour of an array of parallel GaN island transistors, referred in this work as a cell, on a single chip as a number of the cells in the array are deactivated to mimic the isolation of non-functioning cells as outlined by the GaN Systems patent Fault Tolerant Design For Large Area Nitride Semiconductor Devices [6]. The 3D thermal simulation tool Atar was used to build the 3D thermal model of the chip which was then linked into the electrical SPICE simulator OptiSPICE using electrical device models from the GaN Systems GS66516T device [7]. This created an electrothermal simulation that is capable of simulating device characteristics such as individual cell voltages, currents, and temperatures in order to determine the effects that deactivating certain 
combinations of individual cells will have on overall device performance.

\subsection{Thesis Outline}

This thesis will begin in Chapter 2 with a brief background on the concepts necessary to understand the research presented in this thesis. Chapter 3 will present the setup of the electrical, thermal, and combined electrothermal simulations along with the correlation of these simulations with previously established data or physical testing results. These correlation exercises will be used to validate the simulations used in the course of the experiments. Chapter 4 will present preliminary simulations that were performed to investigate the effects of deactivating transistors in the array and the results of those simulations. Finally, Chapter 5 will conclude with a summary of the thesis, the contributions to the objective, and suggestions for the future possibilities of the work. 


\section{Chapter 2}

\section{Background}

\section{$2.1 \quad$ Semiconductors}

In this section there is a brief discussion of the semiconductor physics involved in the operation of the devices used in this thesis, starting from the fundamentals of energy band diagrams, homojunctions, and metal-oxide-semiconductor field-effect transistor (MOSFET) devices and leading into heterojunctions and gallium nitride (GaN) high electron mobility transistors (HEMTs).

\subsubsection{Semiconductor Fundamentals}

In order to understand the formation of the conducting channel in HEMT devices and it's importance to the high power devices covered in this thesis, it is first necessary to understand the basics of the energy band model, which will be illustrated in relation to the most basic interface of two materials, the silicon $p$ - $n$ junction, and the formation of the conducting channel in a MOSFET. 


\section{Energy Band Model}

There are two important models used for the analysis of semiconductor materials: the bonding model and the energy band model. The bonding model depicts the physical arrangement of the atoms and their bonds, and the energy band model describes energy levels and the behaviour of electrons and holes [8]. The energy band diagram is the most useful model for visualizing the behaviour of carriers and therefore it will be the model used throughout the background.

The basic energy band model is a plot of allowed energy states as a function of position as shown in Figure 2.1. The upper band of energy states is the conduction band while the lower band of energy states is the valence band. The energy gap separating the two bands is called the forbidden band, or band gap. The lowest possible conduction band energy is denoted by $E_{c}$, while the highest possible valance band energy is denoted by $E_{v}$. The band gap energy is represented as $E_{g}=E_{c}-E_{v}$. The Fermi level $\left(E_{F}\right)$ represents the electron chemical potential and describes the top of the collection of electron energy levels at absolute zero temperature [9]. The Fermi level can also be viewed as a material property that is related to the concentration of charge carriers that contribute to electrical conduction [10, p. 384].

In an intrinsic (undoped) semiconductor, the concentration of holes in the valence band $(p)$ is equal to the concentration of electrons in the conduction band $(n)$. Setting Equations (2.1) and (2.2) [8, p. 45] equal to each other and solving for $E_{F}$ gives the result shown in Equation (2.3). This result shows that the Fermi energy lies approximately at mid-gap in intrinsic semiconductors $\left(\left(E_{v}+E_{c}\right) / 2 \pm\right.$ a small correction factor that is a linear function of temperature). This Fermi level for intrinsic material is also labelled $E_{i}$ when the Fermi level differs, such as in the case of doped semiconductors. 


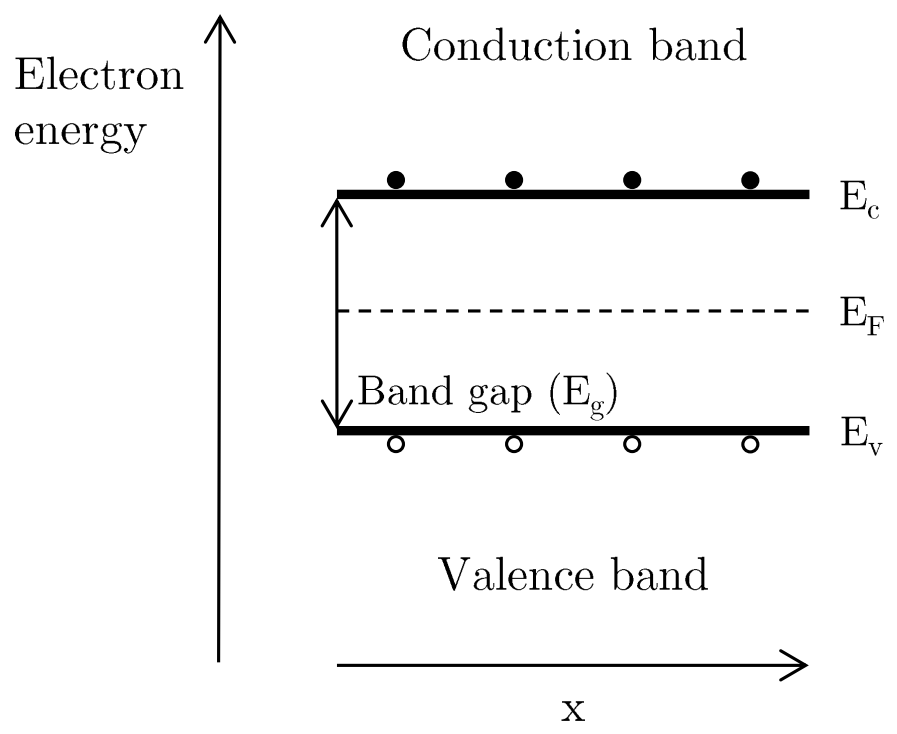

Figure 2.1: Basic energy band diagram.

$$
\begin{aligned}
& n=N_{c} e^{-\left(E_{c}-E_{F}\right) / k T} \\
& p=N_{v} e^{-\left(E_{F}-E_{v}\right) / k T}
\end{aligned}
$$

$$
\begin{aligned}
N_{c} e^{-\left(E_{c}-E_{F}\right) / k T} & =N_{v} e^{-\left(E_{F}-E_{v}\right) / k T} \\
\frac{N_{c}}{N_{v}} & =\frac{e^{-\left(E_{F}-E_{v}\right) / k T}}{e^{-\left(E_{c}-E_{F}\right) / k T}} \\
\ln \left(\frac{N_{c}}{N_{v}}\right) & =-\left(E_{F}-E_{v}\right) / k T+\left(E_{c}-E_{F}\right) / k T \\
k T \ln \left(\frac{N_{c}}{N_{v}}\right) & =E_{v}+E_{c}-2 E_{F} \\
E_{F} & =\frac{E_{v}+E_{c}}{2}-\frac{k T}{2} \ln \left(\frac{N_{c}}{N_{v}}\right)
\end{aligned}
$$

When semiconductors are doped, this causes the Fermi level to shift closer to $E_{c}$ 
for an $n$-type dopant, and closer to $E_{v}$ for a $p$-type dopant. Figure 2.2 shows an example of the position of the Fermi level in doped semiconductors.

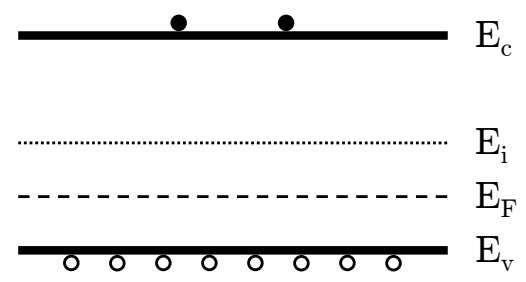

(a) $p$-type

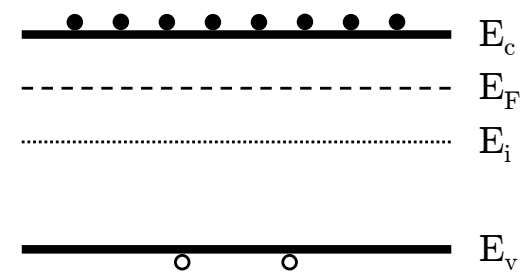

(b) n-type

Figure 2.2: Energy band diagram showing the Fermi level position in doped semiconductors.

\section{Homojunctions}

A homojunction is defined by the semiconductors on either side of a junction having the same material and therefore the same band gap. To illustrate band bending in a homojunction, a basic $p-n$ junction in silicon is considered. Since the silicon is doped $p$-type on one side and $n$-type on the other, when the bands make contact, free electrons from the $n$-type side diffuse into the $p$-type side and free holes diffuse from $p$ to $n$. As the diffusion occurs, the carriers recombine depleting the region near the interface of mobile charge carriers. This leaves the impurities that were doped into the silicon ionized, creating an electric field that opposes the diffusion until equilibrium is attained.

Band bending will occur as the Fermi energy levels align to reach thermal equilibrium. As there is no difference between the conduction and valence bands, no discontinuities exist as illustrated in Figure 2.3a. The band bending accounts for the variation of electron and hole concentrations in the region around the interface and also the effect of the built-in field which corresponds to a built-in potential [10, p. 495]. This built-in potential serves as a barrier for the movement of both electrons and holes. 
When the $p$ - $n$ junction is forward biased, the bands shift with the applied voltage. The majority of the voltage drops across the depletion region, reducing the builtin potential [11] and causing diffusion to occur again. As electrons and holes can continually be supplied by the voltage source, a current will flow between the two terminals as shown in Figure 2.3b.

\section{MOSFET Channel Formation}

Examining the energy band diagram through the gate of the MOSFET shows how the channel is formed at the oxide-semiconductor interface. The MOSFET channel can be of either $n$-type or $p$-type and MOSFET operation can be characterized in two different ways: normally-off (enhancement mode) and normally-on (depletion mode). The material presented in this section will only look at the operation of $n$-channel enhancement mode devices as an example of the channel formation.

At equilibrium the Fermi levels of the gate and substrate line up. This causes the bands of the material with free carriers to bend depending on the Fermi level differences between the gate and substrate. When the gate voltage is equal to the flat band voltage, the bands no longer bend and there is no depletion region that exists under the gate as shown in Figure 2.4a.

When the gate voltage is between the flat band voltage and the threshold voltage, the MOSFET enters depletion mode where the area under the gate is depleted of mobile majority carriers (holes in the $p$ substrate on an $n$-channel device). For the case depicted in Figure 2.4b, electrons from the drain and source diffuse into the bulk and recombine with the free holes. Electrons that have not recombined begin to accumulate at the interface under the gate.

When the gate voltage exceeds the threshold voltage, the intrinsic Fermi energy level shifts below the Fermi level of the substrate by $\phi_{b}$, the bulk potential. The type of the substrate 'inverts' ( $p$-type inverts to $n$-type) and the density of electrons that have 


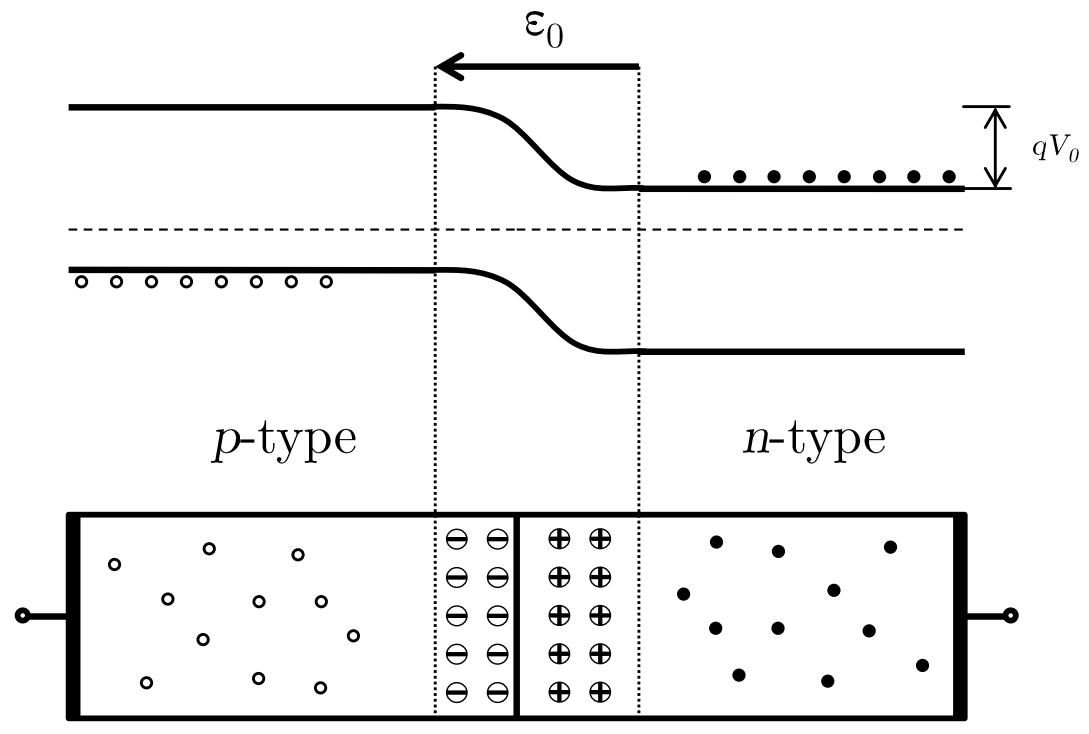

(a) $p$ - $n$ Junction at Equilibrium

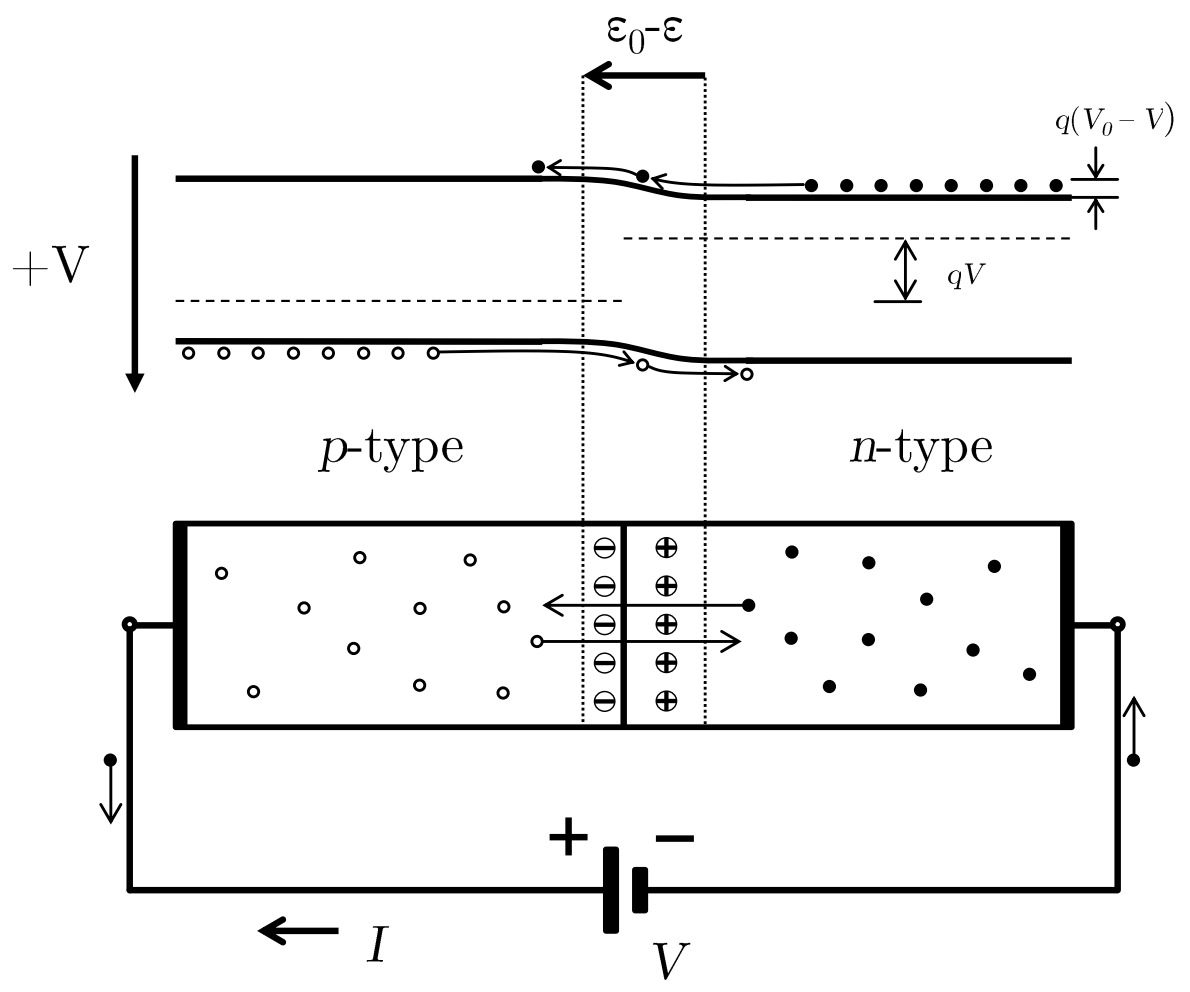

(b) $p$ - $n$ Junction at Forward Bias

Figure 2.3: Energy band diagrams of a $p-n$ junction at equilibrium and at forward bias. 
been accumulating at the interface are enough to form a conducting channel, called the inversion layer, between the source and the drain. Electrons in this channel are free to move in all three dimensions and are subject to scattering effects such as ionized impurity scattering, surface roughness scattering, and thermal lattice scattering [12]. This scattering leads to reduced carrier mobility and is one of the limiting factors for high current/high power silicon MOSFET devices.

\subsubsection{High Electron Mobility Transistors}

Building on the energy band model concepts presented in Section 2.1.1, the operation of the HEMT can be described in terms of the formation of the two dimensional electron gas (2DEG) as can be illustrated on the band diagram. HEMTs differ from MOSFETs primarily in the conducting channel, which is confined to two dimensions rather than the three dimensional inversion layer formed in the MOSFET. This confinement leads to a higher carrier mobility and velocity as a result of reduced ionized impurity scattering due to spatial separation of electrons and ionized impurities [13]. This leads to HEMT devices being capable of having a higher current density than MOSFET devices. Much like the case of MOSFET operation, HEMT devices are characterized as either enhancement or depletion mode devices and material presented in this section will only concern the operation of enhancement devices (e-HEMTs).

\section{Heterojunctions and the Two Dimensional Electron Gas}

Unlike the silicon $p$ - $n$ junction (homojunction), the semiconductors on each side of the heterojunction interface are different materials and therefore have different band gaps. This difference in band gaps causes discontinuities to occur in the valence and conduction bands when the two materials are brought into contact with each other. Heterojunctions are classified into three different types depending on the relative levels of the valence and conduction bands between the two different semiconducting 

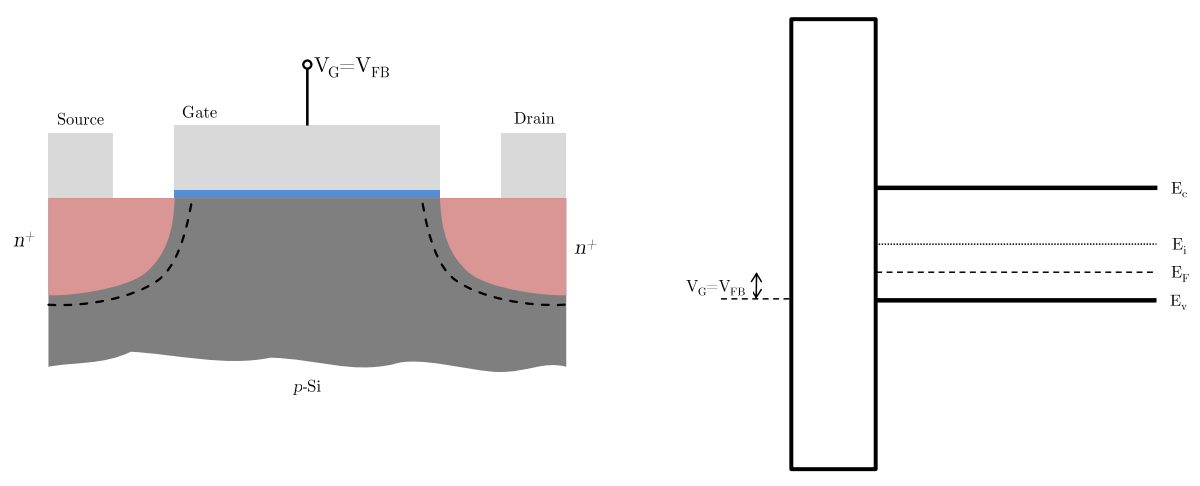

(a) Flat band
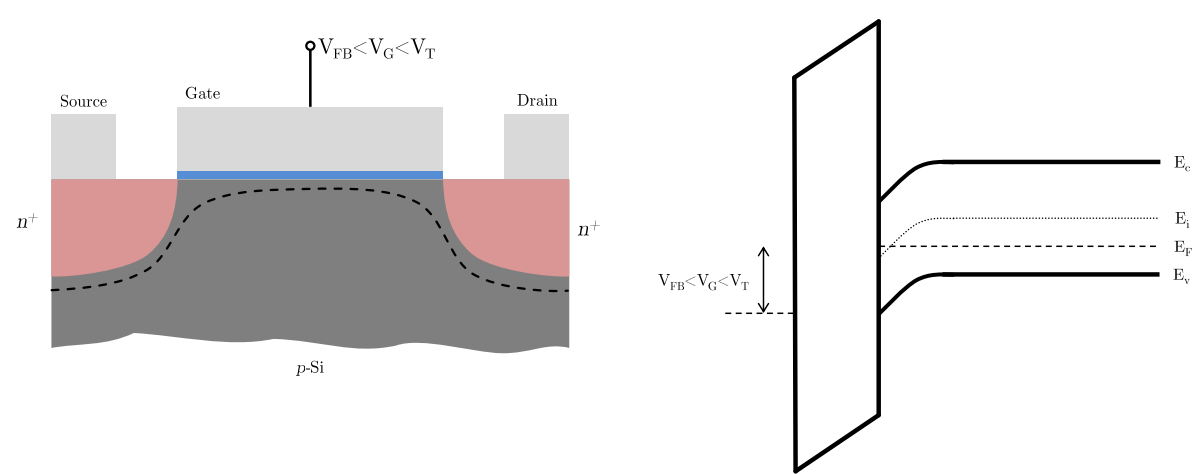

(b) Depletion
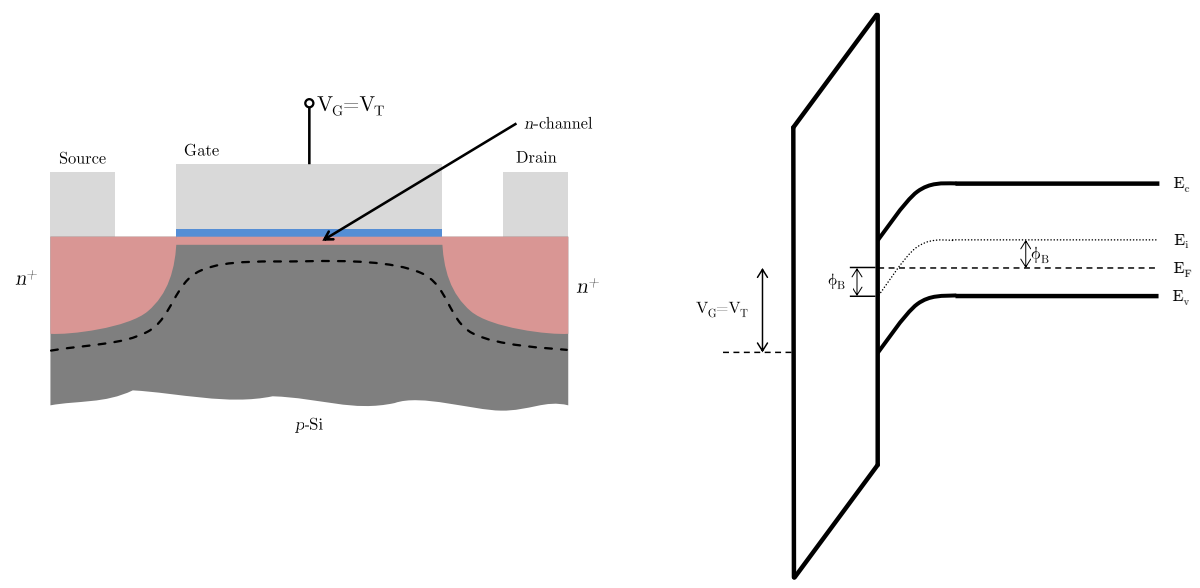

(c) Inversion

Figure 2.4: Cross-section and energy band diagrams showing band bending in metal-oxide-semiconductors. 
materials: straddling, staggered, and broken.

When the band gap of one material is contained within the band gap of the other material, the junction is considered a straddling gap (Type I). The sign conventions for the differences in conduction and valence band energy levels are considered to both be positive for Type I $\left(\Delta \mathrm{E}_{\mathrm{c}}, \Delta \mathrm{E}_{\mathrm{v}}>0\right)[14, \mathrm{p} .86]$. When only one band in each material lies within the band gap of the other material, the junction is considered a staggered gap (Type II). When the band gaps of the two materials do not overlap, the junction is considered a broken gap (Type III). The structures of the three different types are illustrated in Figure 2.5. The devices considered by this thesis are of the straddling gap type and therefore discussion will be limited to only Type I heterojunctions.

When the bands of the heterojunction are brought into contact with each other, the system will shift to reach equilibrium just like in the case of the $p$ - $n$ junction and the MOSFET. Due to the differing band gaps of the two materials, the bands will bend and form discontinuities as shown in Figure 2.6. The exact shape of the bent bands depend on many factors such as doping, mobile charge carriers, Fermi level differences, and electric fields present [15].

The conduction band level in relation to the Fermi level controls the formation of the 2DEG. If the lowest point of the conduction band edge remains above the Fermi level at equilibrium, the 2DEG channel will not form and the device will be considered an enhancement mode device. When a positive voltage above some threshold is applied to the gate (for example, the devices used in this thesis have a threshold voltage in the range of $1.1 \mathrm{~V}$ to $1.3 \mathrm{~V}$ ), the conduction band drops below the Fermi level and the electrons gather at quantized energy levels in the triangular potential well formed by the bent bands and heterojunction discontinuity as illustrated in Figure 2.7. This quantum well will confine the movement of electrons to two directions while restricting movement in the third direction [16, p. 58]. This forms a two dimensional sheet of electrons parallel to the heterointerface know as the 2DEG. 


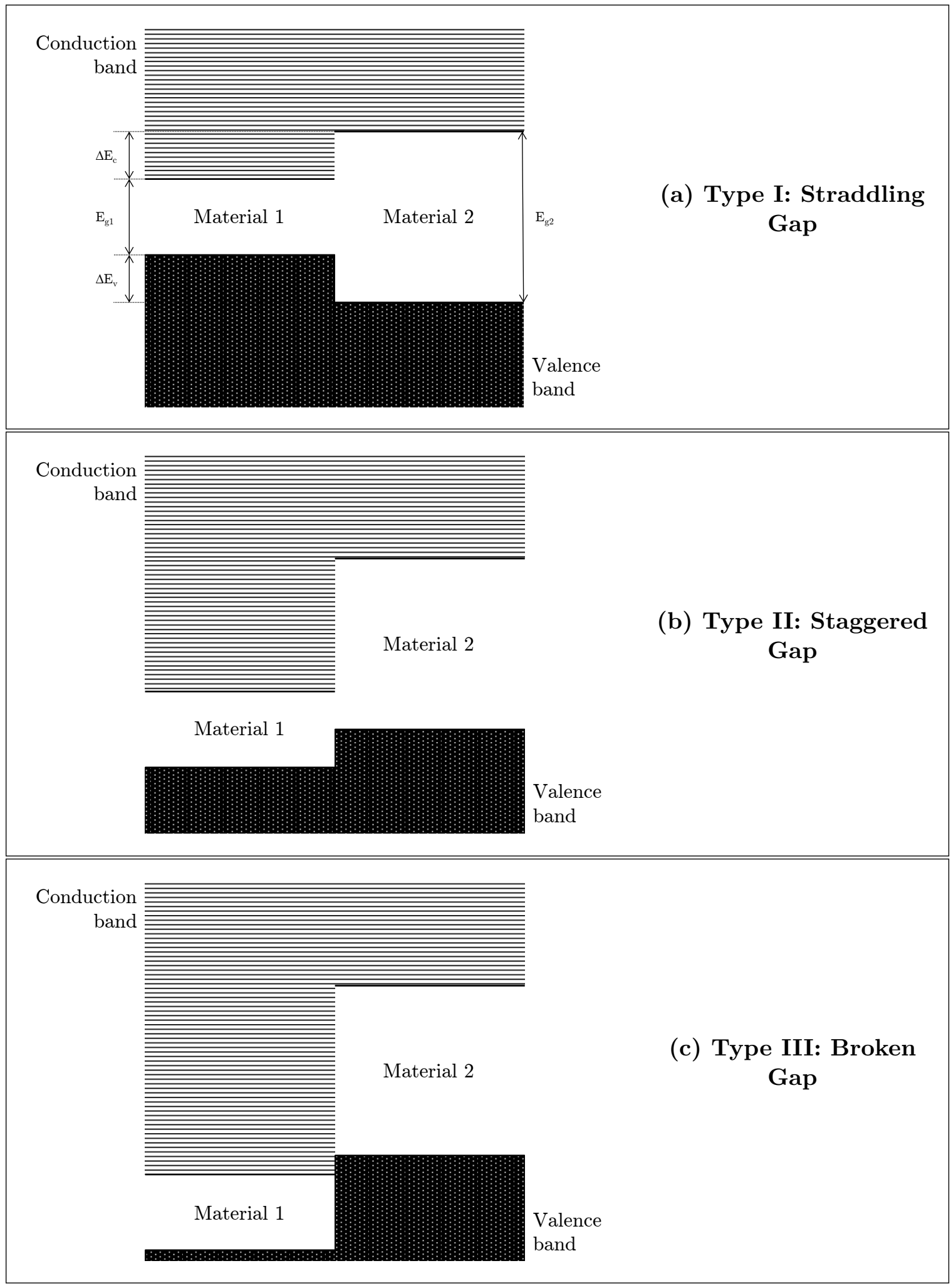

Figure 2.5: Energy band diagrams showing the three different types of heterojunction interfaces: straddling gap, staggered gap, and broken gap. 


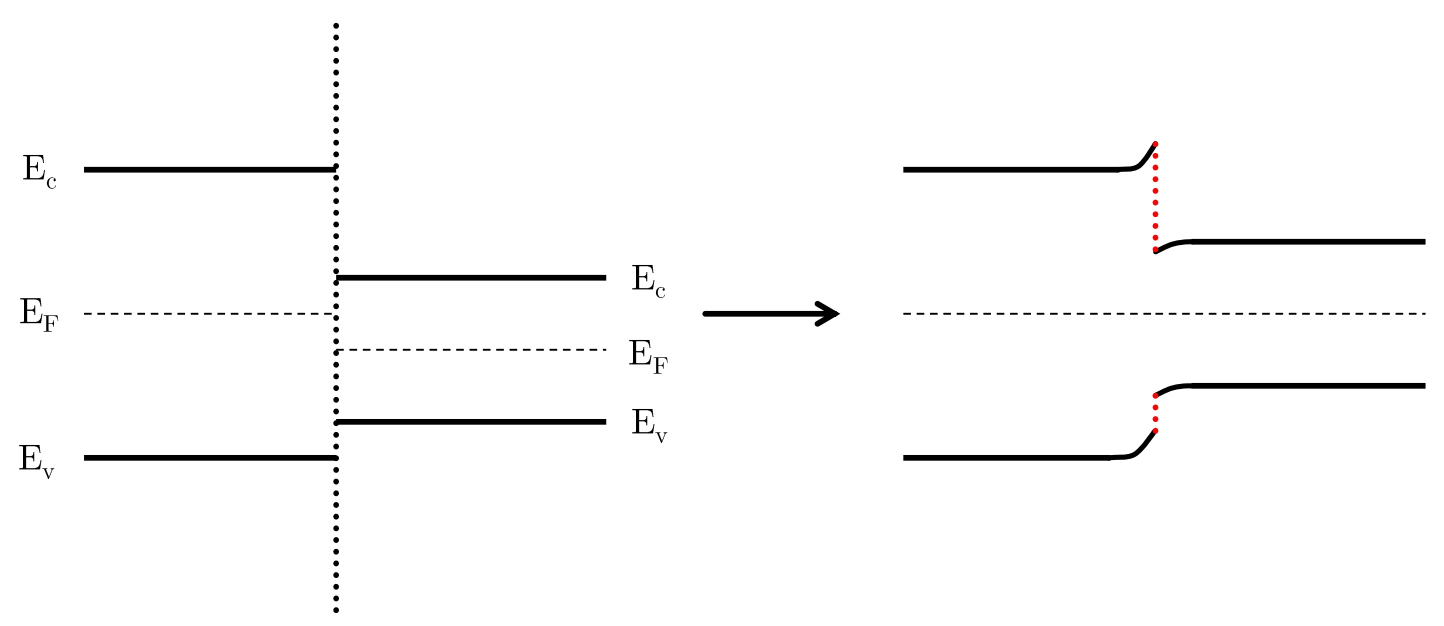

Figure 2.6: Energy band diagram of a straddling gap heterojunction showing band bending.

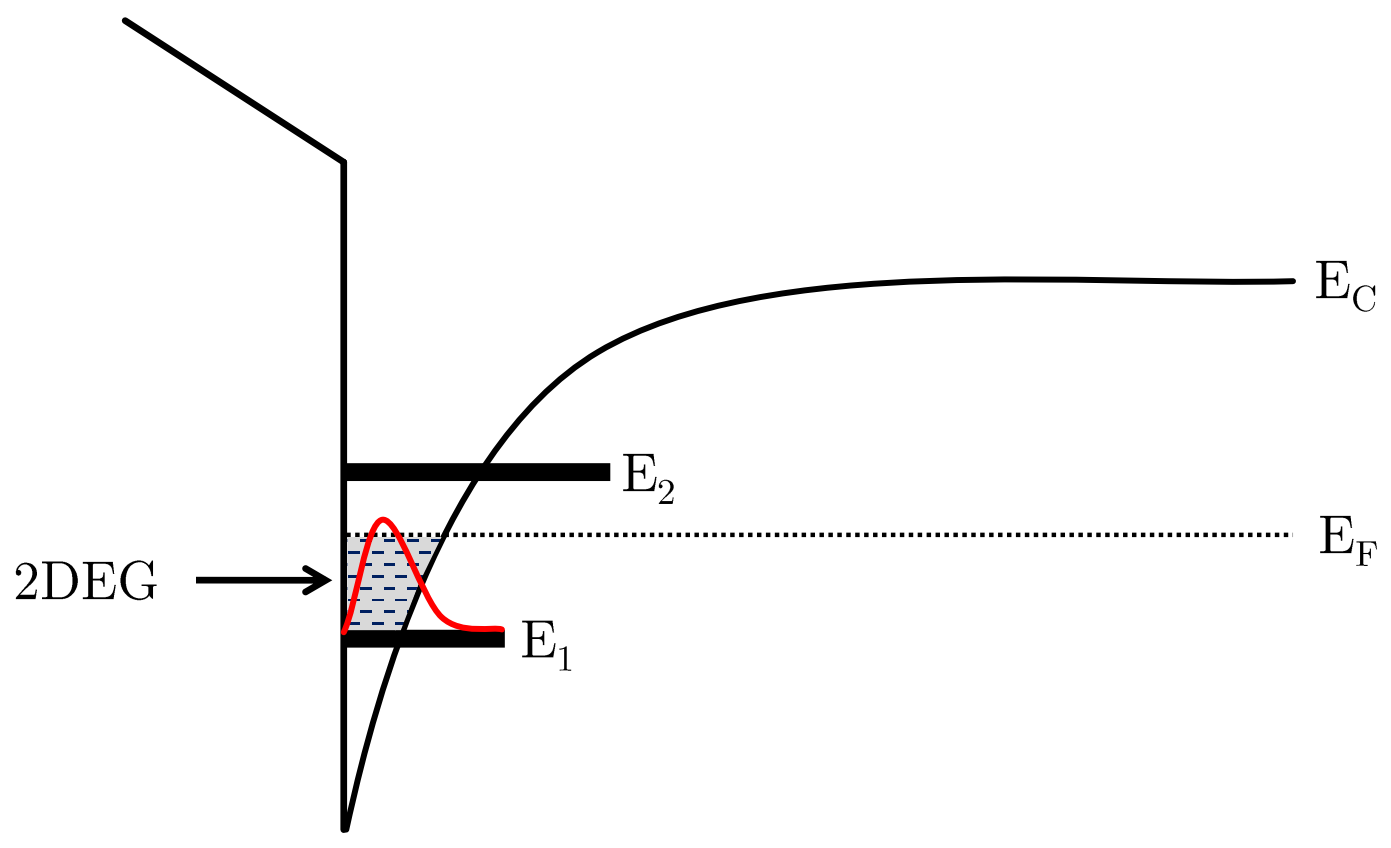

Figure 2.7: Energy band diagram of a heterojunction showing the formation of the 2DEG. 


\section{Gallium Nitride HEMTs}

GaN undergoes both spontaneous polarization due to its crystal structure and piezoelectric polarization when it undergoes stress or strain. The alloys of GaN, like AlGaN, exhibit the same spontaneous and piezoelectric polarization behaviour. This has the benefit that, unlike conventional HEMT devices (such as AlGaAs/GaAs), aluminium gallium nitride $(\mathrm{AlGaN}) / \mathrm{GaN}$ does not require intentional doping to generate the 2DEG. It has been shown by Ibbetson et al [17] and confirmed by He et al [18] that the electrons in the 2DEG come from donor states on the AlGaN surface.

The lattice mismatch between the $\mathrm{AlGaN}$ and the GaN causes a piezoelectric polarization due to induced strain in the thin AlGaN layer. This adds to the spontaneous polarization already present as shown in Figure 2.8 and results in a high electric field in the AlGaN layer. If the AlGaN layer is sufficiently thick, the Fermi level will reach the donor state level, $E_{S}$, and electrons from the surface donor states will be promoted into the conduction band and move under the force of the polarizationinduced electric field to the $\mathrm{AlGaN} / \mathrm{GaN}$ interface. At the interface, the electron will move to the lower GaN conduction band where they become trapped in the potential well and form the 2DEG [18]. An illustration of the electron movement from the surface donor states in freestanding AlGaN is shown in Figure 2.9a and the electron transfer to the GaN layer forming the 2DEG in Figure 2.9b. The areal concentration of electrons in the 2DEG, $n_{s}$, can be as high as $10^{13} \mathrm{~cm}^{-2}$ which gives AlGaN/GaN HEMTs a high current carrying capability [19].

A typical AlGaN/GaN e-HEMT structure (including the location of the 2DEG) is shown in Figure 2.10. Benefits of using undoped AlGaN include a reduction in gate leakage current [20] and benefits of using undoped $\mathrm{GaN}$ include a reduction in ionized impurity scattering [13] leading to a higher mobility in the 2DEG. 


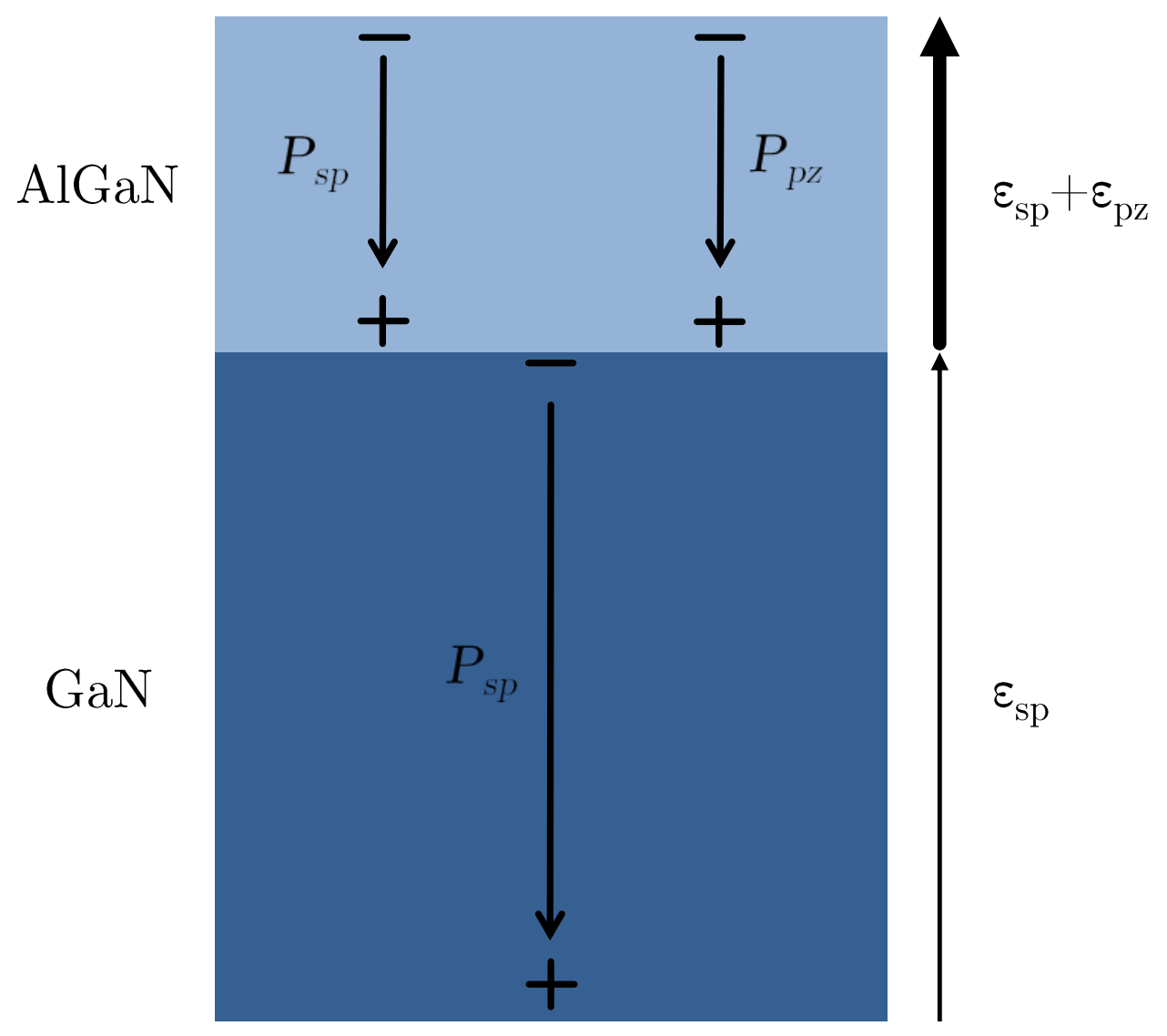

Figure 2.8: Location and direction of polarization and electric fields in GaN and strained AlGaN. 


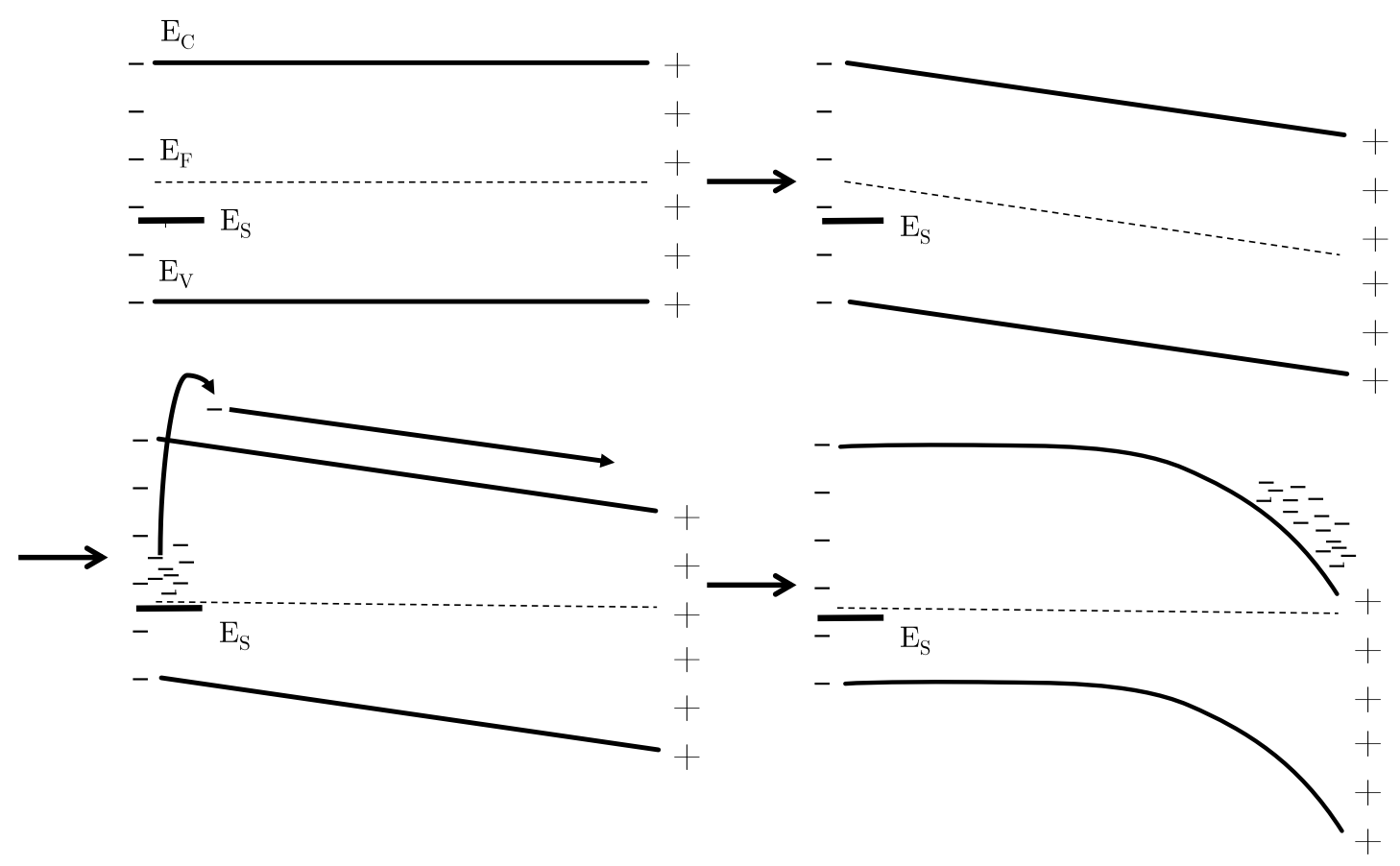

(a) Freestanding AlGaN energy band diagram showing the movement of electrons from the surface states under effect of the polarization-induced electric field.

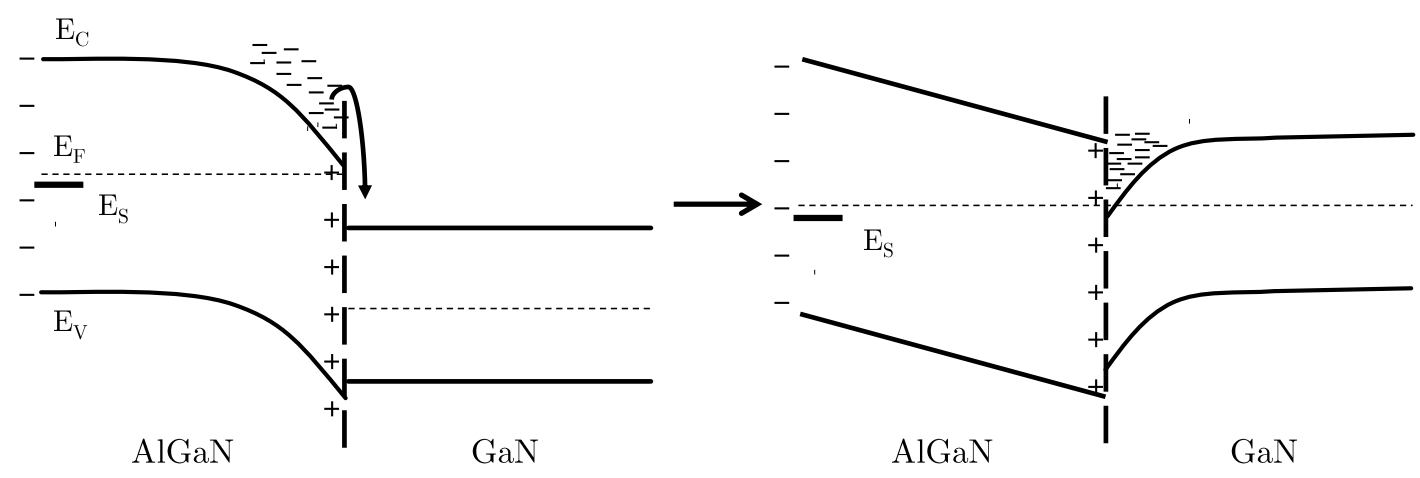

(b) Undoped AlGaN/GaN heterostructure showing the formation of the 2DEG as the electrons flow into the GaN side and accumulate at the interface.

Figure 2.9: The movement of electrons from the surface donor states and the formation of the 2DEG at the heterointerface in an AlGaN/GaN heterostructure (adapted from [18]). 


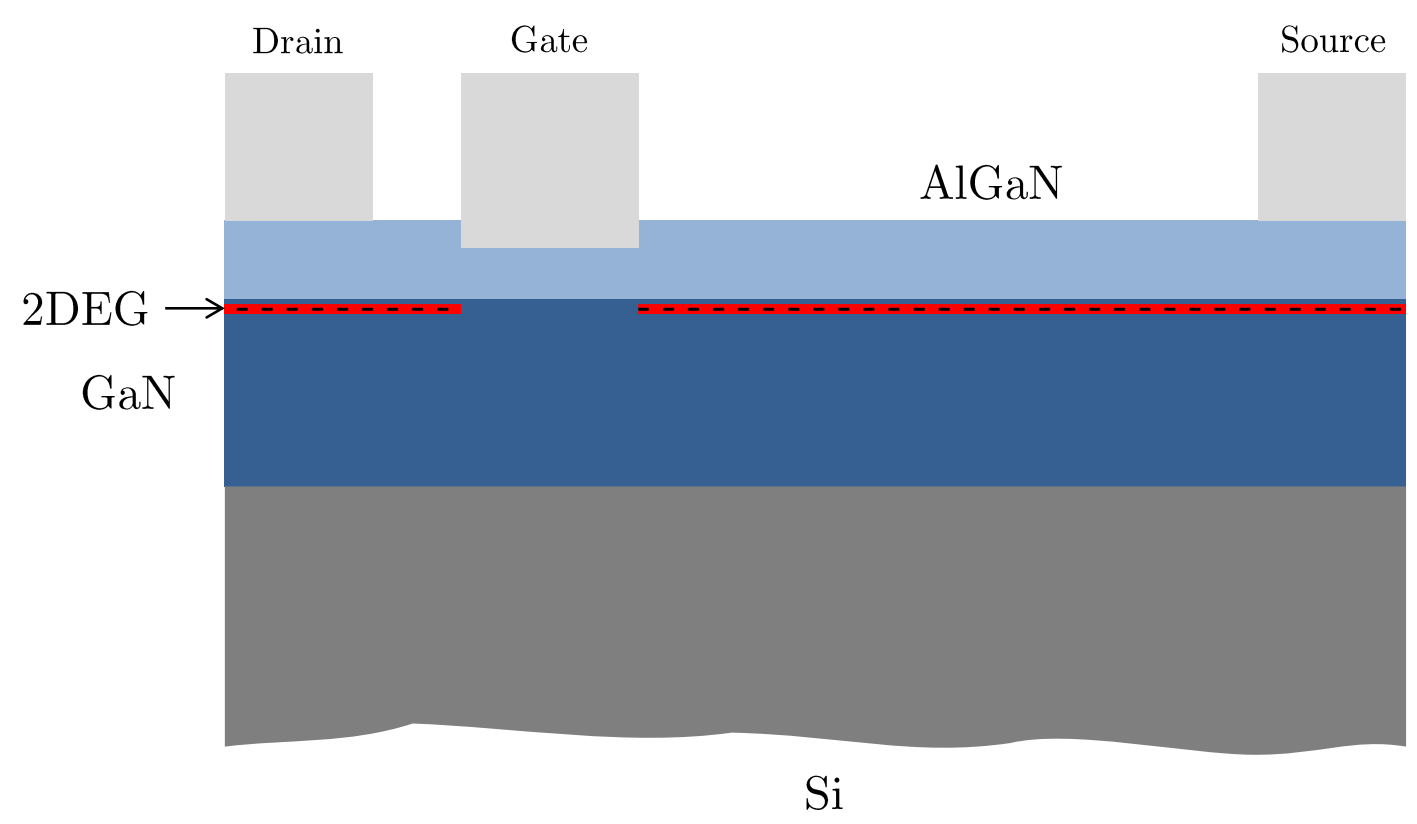

Figure 2.10: Cross-section diagram of a typical GaN eHEMT structure.

\subsection{Thermodynamics}

This section will deal with the concepts of thermodynamics involved in this thesis which are primarily used in simulations, but also in the physical testing of the devices.

\subsubsection{Heat Transfer Methods}

The three primary methods of heat transfer are conduction, convection, and radiation. Conduction is the process of heat diffusing through a solid or stationary fluid and is governed by Fourier's Law and the Heat Equation as discussed in the following section.

Convection is the heat transfer from a surface assisted by the motion of a fluid (including air). Heat transfer by convection is represented by Equation (2.4) where $T$ is the surface temperature, $T_{\infty}$ is the external ambient temperature, and $h$ is the heat transfer coefficient in $\mathrm{W} / \mathrm{m}^{2} \mathrm{~K}$. The heat transfer coefficient depends on the type of flow, geometry of the body and flow passage area, the physical properties of the 
fluid, the average temperature, and more [21].

$$
\mathbf{q}_{c o n v}=h\left(T-T_{\infty}\right)
$$

Radiation is the exchange of heat between surfaces, or between a surface and a surrounding fluid, by electromagnetic radiation. This takes the form of Equation (2.5) where $T$ is again the surface temperature, $T_{r}$ is the ambient effective temperature, $\epsilon$ is the emissivity of the surface, and $\sigma$ is the Stefan-Boltzmann constant in $\mathrm{W} / \mathrm{m}^{2} \mathrm{~K}^{4}$.

$$
\mathbf{q}_{r a d}=\epsilon \sigma\left(T^{4}-T_{r}^{4}\right)
$$

Due to the non-linearity of radiation effects and the many different models for convection, only the effects of conduction were considered to simplify the thermal simulations performed in this thesis.

\subsubsection{Fourier's Law and the Heat Equation}

Fourier's Law describes the relationship between heat flow and temperature gradient as shown in Equation (2.6) where $\mathbf{q}_{n}$ is the heat transfer rate in the $n$ direction in $\mathrm{W} / \mathrm{m}^{2}, \kappa_{n}$ is the thermal conductivity in the $n$ direction in $\mathrm{W} / \mathrm{mK}$, and $\partial T / \partial n$ is the temperature gradient in the $n$ direction in $\mathrm{K} / \mathrm{m}$. The negative sign is added to ensure the heat flow is represented as a positive quantity due to the temperature gradient being negative $[22$, p. 2]. Though in general the thermal conductivity is a function of both temperature and direction $(\kappa(n, T))$, it was assumed for this thesis that thermal conductivity was temperature independent and only directionally dependent in special cases.

$$
\mathbf{q}_{n}=-\kappa_{n} \frac{\partial T}{\partial n}
$$

Fourier's Law can also be used to define a conduction thermal resistance in $\mathrm{K} / \mathrm{W}$ 
as in Equation (2.7) by relating the temperature difference, $T_{1}-T_{2}$, to the thermal conductivity of the material, the cross-sectional area, $A$, and the path length, $L[22$, p. 2].

$$
R_{\theta}=\frac{\left(T_{1}-T_{2}\right)}{q}=\frac{L}{\kappa A}
$$

The general heat conduction equation is a partial differential equation that describes the temperature distribution in a stationary medium over time and can be derived from the energy balance shown in Equation (2.8).

$$
\left[\begin{array}{c}
\text { Rate of heat entering through } \\
\text { the bounding surfaces of } V
\end{array}\right]+\left[\begin{array}{l}
\text { Rate of energy } \\
\text { generation in } V
\end{array}\right]=\left[\begin{array}{c}
\text { Rate of energy } \\
\text { storage in } V
\end{array}\right]
$$

Following the derivation presented in detail by Ozisik [21], the heat conduction equation is shown in Equation (2.9) where the rate of heat entering through the bounding surfaces is represented by the second order derivatives of temperature and thermal conductivities, the rate of energy generation is represented by $\mathbf{g}$ in $\mathrm{W} / \mathrm{m}^{3}$ and the rate of energy storage is given by $\rho C_{p} \frac{\partial T}{\partial t}$ in which $\rho$ is the density of the material in $\mathrm{kg} / \mathrm{m}^{3}$, and $c_{p}$ is the specific heat of the material in $\mathrm{J} / \mathrm{kg} \cdot \mathrm{K}$. The thermal conductivity is inside the derivative for cases of conductivities that are directionally dependant.

$$
\frac{\partial}{\partial x}\left(\kappa \frac{\partial T}{\partial x}\right)+\frac{\partial}{\partial y}\left(\kappa \frac{\partial T}{\partial y}\right)+\frac{\partial}{\partial z}\left(\kappa \frac{\partial T}{\partial z}\right)+\mathbf{g}=\rho c_{p} \frac{\partial T}{\partial t}
$$

The change in thermal energy storage, $\rho C_{p} \frac{\partial T}{\partial t}$, goes to zero at steady-state giving the steady-state heat conduction equation as shown in Equation (2.10).

$$
\frac{\partial}{\partial x}\left(\kappa \frac{\partial T}{\partial x}\right)+\frac{\partial}{\partial y}\left(\kappa \frac{\partial T}{\partial y}\right)+\frac{\partial}{\partial z}\left(\kappa \frac{\partial T}{\partial z}\right)+\mathbf{g}=0
$$

Simulations in this thesis only concern steady-state conduction but transient analysis is something that would prove useful for future consideration. 


\subsubsection{Boundary Conditions}

To solve the heat conduction equation, boundary conditions must be specified. As the equation is second order in space, two boundary conditions must be specified for each dimension. The three common boundary conditions encountered are constant surface temperature, constant surface flux, and a relationship between surface heat flux and surface temperature [22, p. 166].

Constant surface temperature, also known as the fixed boundary condition, allows for the setting of a fixed temperature at a boundary as in Equation(2.11).

$$
T(0)=T_{B C}
$$

Constant surface flux is where the heat flux at a surface is specified $\left(q_{s}\right.$ in $\left.\mathrm{W} / \mathrm{m}^{2}\right)$ as shown in Equation (2.12). This is useful for defining a generating region at a surface.

$$
-\left.\kappa \frac{\partial T}{\partial x}\right|_{x=0}=q_{s}
$$

A special case of the constant surface flux condition is when surface flux equals zero as shown in Equation (2.13). This corresponds to a perfectly insulated, or adiabatic, surface with no heat flow. This behaves like free space, ignores the effects of radiation and convection, and can act as a thermal mirror [23].

$$
\left.\frac{\partial T}{\partial x}\right|_{x=0}=0
$$

The final common boundary condition, the relationship between surface heat flux and surface temperature, is a mixed boundary condition and can mimic the effects of convection as shown in Equation (2.14).

$$
-\left.\kappa \frac{\partial T}{\partial x}\right|_{x=0}=h\left[T_{\infty}-T(0)\right]
$$


Only the fixed temperature, constant flux, and the insulating boundary conditions were considered in the simulations used in the course of this thesis. The effects of convection and radiation were ignored in order to simplify the solutions. This simplification should still provide accurate results as the devices should not get hot enough for radiation to have much of an effect and the devices that were modelled are encased in a packaging material which would negate any convection effects. In the future, a model of the device including the package and taking into account the effects of radiation and convection could be investigated to confirm the accuracy of the simulations performed in this thesis.

\subsection{Thermal Effects in GaN}

There are three major mechanisms responsible for heating in semiconductor devices: Joule heating, carrier generation and recombination heating, and thermoelectric effects [24]. Joule heating is the dominant cause of self-heating due to the high current density and electric fields present in the 2DEG channel in HEMT devices [25]. Joule heating, also known as Ohmic or resistive heating, is heat generated by the current flow through any resistive material. This generated heat arises from the power dissipated through any active or passive device. The power dissipated can be calculated using Joule's Law (Equation (2.15)) [26].

$$
P=I^{2} R=I V
$$

The contribution from both gate and drain constitute the full power dissipation of the GaN device: $P_{D}=I_{G S} V_{G}+I_{D S} V_{D S}$. Due to the large drain-source current dominating over any gate leakage current, the power dissipated can be simplified to $P_{D}=I_{D S} V_{D S}$. As the power is dissipated primarily in the 2DEG channel, this can give rise to large junction temperatures which can have adverse effects on the 
operation and lifetime of the devices.

\subsubsection{Recoverable Effects}

Recoverable effects are those which have no memory, the effects revert back to normal when the device returns to an inactive state.

\section{Electron Mobility Degradation}

It can be seen in the example $I_{D S} V_{D S}$ curves for an AlGaN/GaN HEMT in Figure 2.11 that the drain current in saturation shows a dependence on the power dissipated by the device when self-heating is taken into account. This is caused by a decrease in electron mobility in the 2DEG as the device heats up [27]. As the voltage increases and the power dissipation becomes larger, the current starts to decrease more due to the increase in temperature. It was found by Tan et al [28] that the temperature dependence of the saturation drain current is $T^{-1.5}$ for long channel devices $(>5 \mu m)$ and $T^{-0.5}$ for short channel devices. For the case of undoped/lightly doped AlGaN/GaN, the primary cause for this decrease in mobility as temperature increases has been determined to be thermal lattice, or phonon, scattering [29].

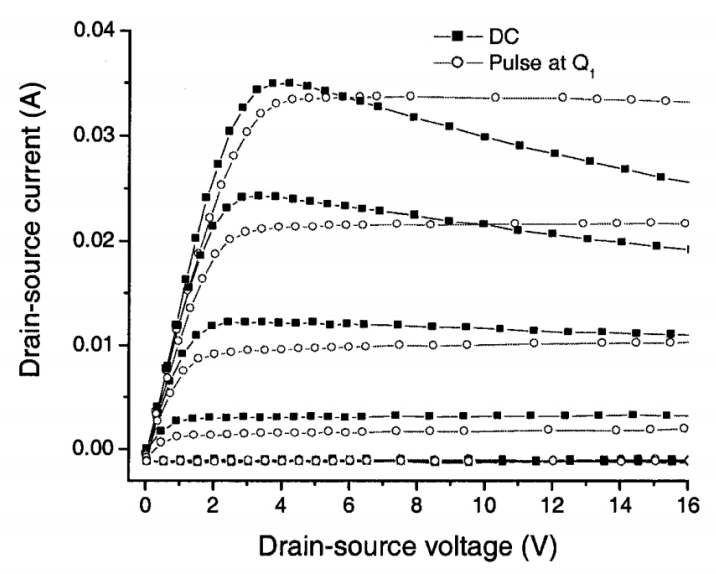

Figure 2.11: AlGaN/GaN DC characteristics with and without self-heating effects (used with permission from [23]). 


\section{RF Effects}

High temperatures in the devices may have an effect on RF performance. Some RF characteristics are negatively influenced by temperature such as increased noise, reduced transistor gain, and reduction in operating frequency [23].

\subsubsection{Permanent Effects}

Permanent effects are those which persist even after the device returns to an inactive state, typically corresponding to damage of some sort.

\section{Crystal Dislocations}

Due to the differing thermal expansion coefficients of the materials present in the HEMT structures and the inverse piezoelectric effect, high temperatures combined with high electric fields [30] may cause fracturing and dislocations at the interfaces between the AlGaN and the GaN. This may introduce traps or dislocation scattering which would impact carrier mobility [31] and decrease device performance in a nonrecoverable manner.

\section{Electromigration}

Electromigration is the process in which metal atoms are forced to migrate by large current density [10, p. 172]. This migration can cause voids and hillocks to occur which can cause breaks or shorts in interconnect layers. The rate of electromigration is related to temperature and current density as shown in Equation (2.16) [10, p. 177] where $J$ is the current density in $\mathrm{A} / \mathrm{m}, E_{A}$ is the activation energy involved in migrating the metal atom in $\mathrm{eV}, k$ is the Boltzmann constant, and $T$ is temperature in K. The high temperatures and current densities that GaN devices are capable of may lead to increased rates of electromigration failures in comparison to other 
technologies.

$$
R_{E M} \propto J^{n} e^{-\frac{E_{A}}{k T}}
$$

\subsection{Electrical and Thermal Simulation}

This section will deal with some of the concepts necessary for the electrical and thermal simulations, as well as a discussion of the simulators considered for use in this thesis.

\subsubsection{SPICE Basics}

Simulation Program with Integrated Circuit Emphasis (SPICE) is an electronics circuit simulation tool that uses modified nodal analysis (MNA) to build up circuit equations from a netlist of circuit elements. It uses 'stamps', device equations in matrix form, of each component to map the netlist into a matrix equation. This final MNA matrix form is shown in Equation (2.17) where $\mathbf{G}$ is the conduction matrix representing linear resistors/relationships, $\mathbf{C}$ is the capacitive matrix representing energy storage elements (capacitors and inductors), $\mathbf{B}$ represents non-linear devices, $\mathbf{F}(t)$ represents independent current/voltage sources, and $x$ is the vector of node voltages, device currents, and charges to be determined.

$$
\mathbf{G} x+\mathbf{C} \dot{x}+\mathbf{B}(x)=\mathbf{F}(t)
$$

A comparison of some SPICE statements and their representative circuit elements and stamps is shown in Figure 2.12. An example circuit is shown in Figure 2.13 with the matrix equation $\mathbf{G} x+\mathbf{C} \dot{x}=\mathbf{F}(t)$. The values of $\mathbf{G}, \mathbf{C}, x, \dot{x}$, and $\mathbf{F}(t)$ shown in Equations (2.18) and (2.19) were derived from the stamps of the elements based on the nodes shown in the example circuit. 


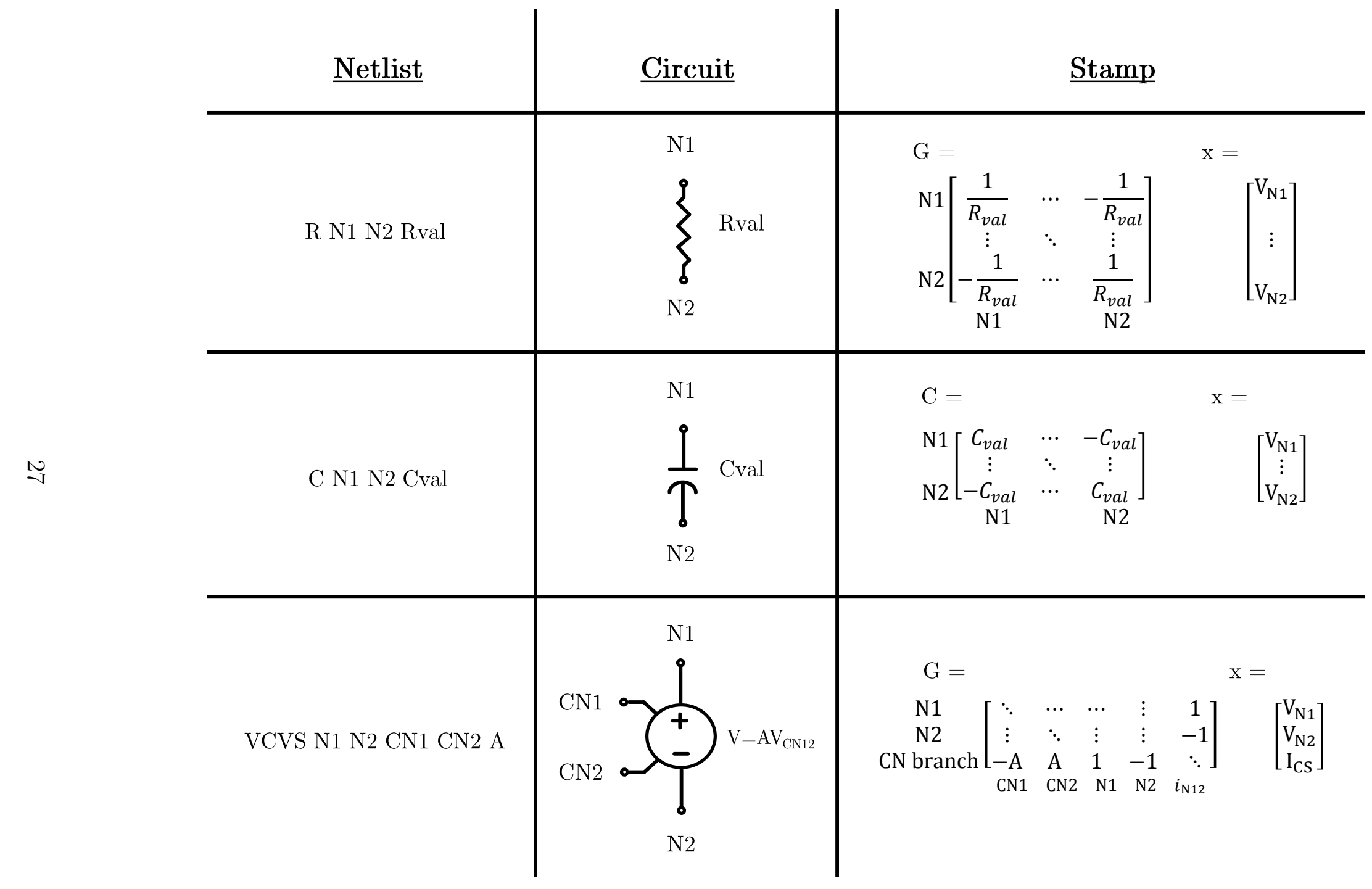

Figure 2.12: A comparison of some SPICE statements, their representative circuit elements, and stamps used to map the netlist into a matrix equation for MNA. 


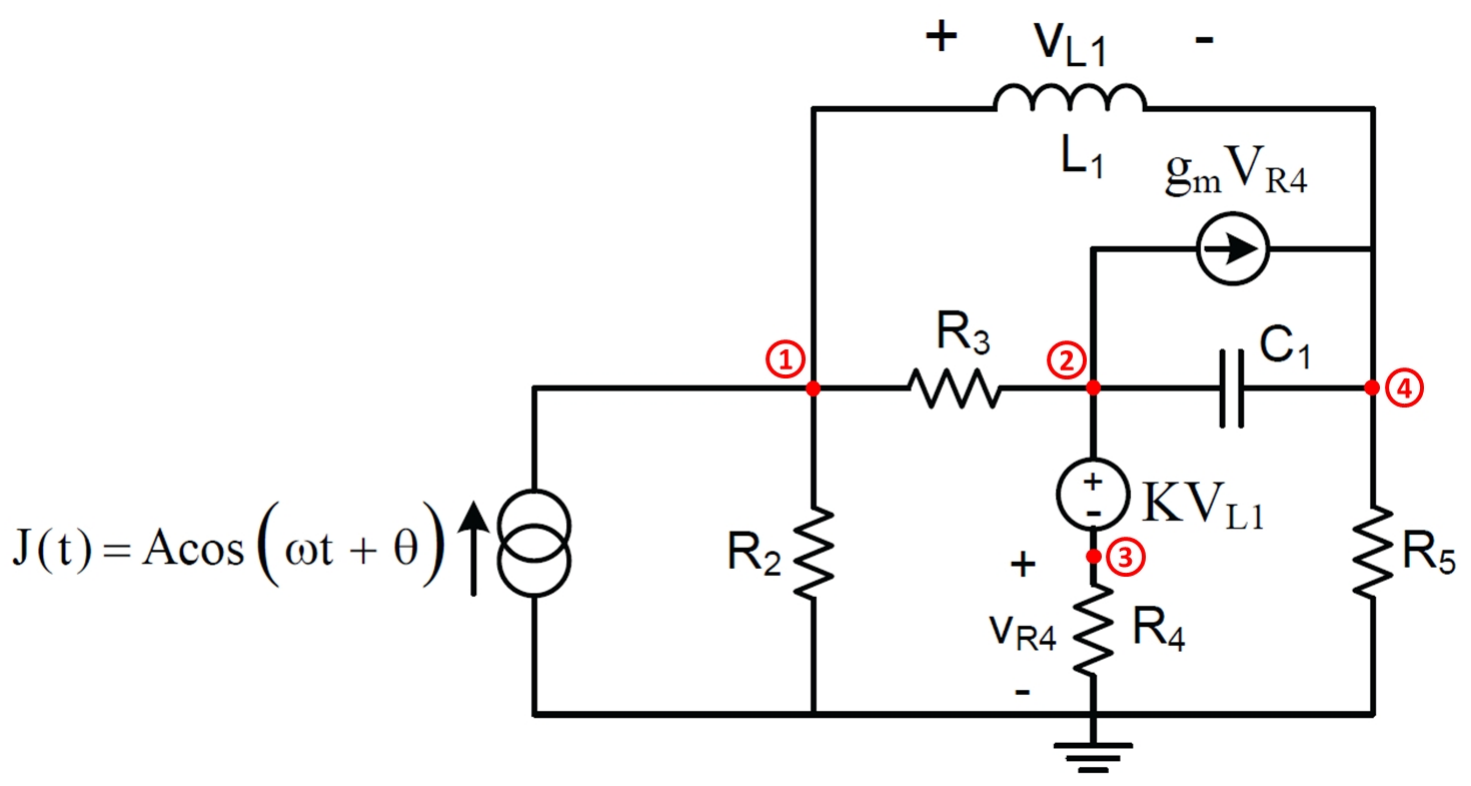

Figure 2.13: Example SPICE circuit used for demonstrating MNA matrix equation derivation.

$$
\mathbf{G}=\left[\begin{array}{cccccc}
\frac{1}{R_{2}}+\frac{1}{R_{3}} & -\frac{1}{R_{3}} & 0 & 0 & 1 & 0 \\
-\frac{1}{R_{3}} & \frac{1}{R_{3}} & g_{m} & 0 & 0 & 1 \\
0 & 0 & \frac{1}{R_{4}} & 0 & 0 & -1 \\
0 & 0 & -g_{m} & \frac{1}{R_{5}} & -1 & 0 \\
1 & 0 & 0 & -1 & 0 & 0 \\
-K & 1 & -1 & K & 0 & 0
\end{array}\right] \quad \mathbf{C}=\left[\begin{array}{cccccc}
0 & 0 & 0 & 0 & 0 & 0 \\
0 & C_{1} & 0 & -C_{1} & 0 & 0 \\
0 & 0 & 0 & 0 & 0 & 0 \\
0 & -C_{1} & 0 & C_{1} & 0 & 0 \\
0 & 0 & 0 & 0 & -L_{1} & 0 \\
0 & 0 & 0 & 0 & 0 & 0
\end{array}\right]
$$

$$
x=\left[\begin{array}{c}
v_{1} \\
v_{2} \\
v_{3} \\
v_{4} \\
i_{L 1} \\
i_{E}
\end{array}\right] \quad \dot{x}=\left[\begin{array}{c}
\dot{v}_{1} \\
\dot{v}_{2} \\
\dot{v_{3}} \\
\dot{v_{4}} \\
\dot{i_{L 1}} \\
\dot{i_{E}}
\end{array}\right] \quad \mathbf{F}(t)=\left[\begin{array}{c}
J(t) \\
0 \\
0 \\
0 \\
0 \\
0
\end{array}\right]=\left[\begin{array}{c}
A \cos (\omega t+\theta) \\
0 \\
0 \\
0 \\
0 \\
0
\end{array}\right]
$$


The SPICE engine can then solve the matrix equation using different numerical methods [32] for different types of analyses such as DC operating point, transient analysis, frequency domain, etc. The SPICE simulations in this thesis were performed for the DC operating point or DC sweep only.

\subsubsection{Finite Difference Method}

The finite difference method is a numerical method for solving differential equations. It works by replacing a differential equation with a difference equation based on the premise that a differential quantity can be approximated accurately using sufficiently small differences as in Equation (2.20).

$$
\frac{d f(x)}{d x}=\lim _{\Delta x \rightarrow 0} \frac{\Delta f(x)}{\Delta x} \approx \frac{f(x+\Delta x)-f(x)}{\Delta x}
$$

The following is an example of the finite difference method for the 2D steady state heat equation in Equation (2.21) for the mesh shown in Figure 2.14.

$$
\frac{\partial^{2} T}{\partial x^{2}}+\frac{\partial^{2} T}{\partial y^{2}}+\frac{g}{\kappa}=0
$$

For the point $(m, n)$, the first derivatives at points $m-\frac{1}{2} \Delta x, m+\frac{1}{2} \Delta x, n-\frac{1}{2} \Delta y$, and $n+\frac{1}{2} \Delta y$ are approximated in Equations (2.22)-(2.25)

$$
\begin{aligned}
& \left.\frac{\partial T}{\partial x}\right|_{m-1 / 2, n} \approx \frac{T_{m, n}-T_{m-1, n}}{\Delta x} \\
& \left.\frac{\partial T}{\partial x}\right|_{m+1 / 2, n} \approx \frac{T_{m+1, n}-T_{m, n}}{\Delta x}
\end{aligned}
$$




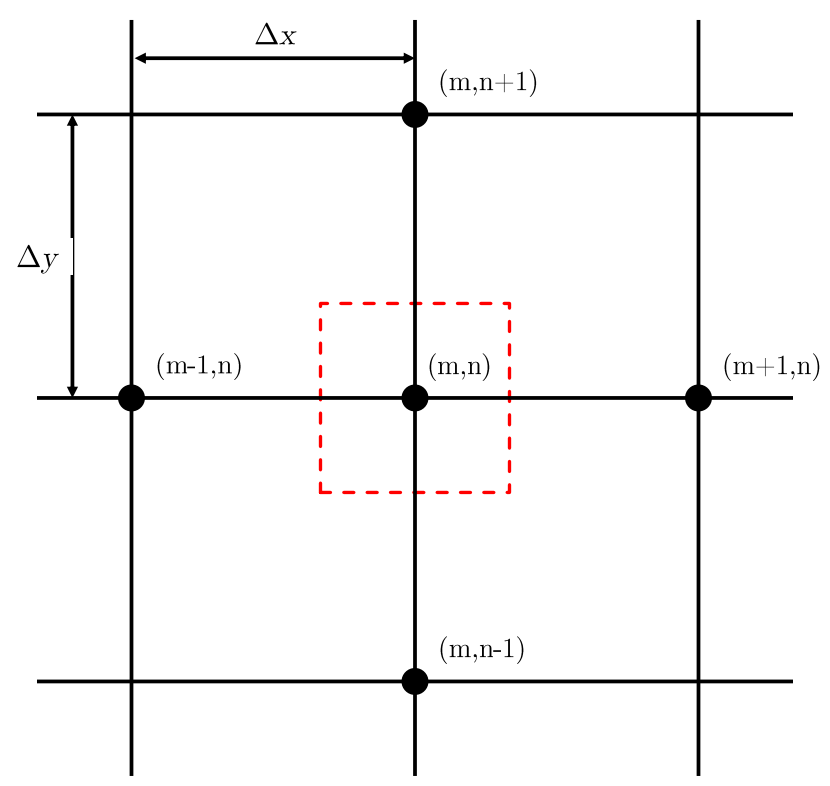

Figure 2.14: Two dimensional finite difference mesh example.

$$
\begin{aligned}
& \left.\frac{\partial T}{\partial y}\right|_{m, n-1 / 2} \approx \frac{T_{m, n}-T_{m, n-1}}{\Delta y} \\
& \left.\frac{\partial T}{\partial y}\right|_{m, n+1 / 2} \approx \frac{T_{m, n+1}-T_{m, n}}{\Delta y}
\end{aligned}
$$

The second derivatives are then approximated as shown in Equations (2.26) and (2.27)

$$
\begin{aligned}
&\left.\frac{\partial^{2} T}{\partial x^{2}}\right|_{m, n} \approx \frac{\left.\frac{\partial T}{\partial x}\right|_{m+1 / 2, n}-\left.\frac{\partial T}{\partial x}\right|_{m-1 / 2, n}}{\Delta x} \approx \frac{\frac{T_{m+1, n}-T_{m, n}}{\Delta x}-\frac{T_{m, n}-T_{m-1, n}}{\Delta x}}{\Delta x} \\
& \approx \frac{T_{m+1, n}-2 T_{m, n}+T_{m-1, n}}{\Delta x^{2}} \\
&\left.\frac{\partial^{2} T}{\partial y^{2}}\right|_{m, n} \approx \frac{\left.\frac{\partial T}{\partial y}\right|_{m, n+1 / 2}-\left.\frac{\partial T}{\partial y}\right|_{m, n-1 / 2}}{\Delta y} \approx \frac{\frac{T_{m, n+1}-T_{m, n}}{\Delta y}-\frac{T_{m, n}-T_{m, n-1}}{\Delta y}}{\Delta y}
\end{aligned}
$$




$$
\approx \frac{T_{m, n+1}-2 T_{m, n}+T_{m, n-1}}{\Delta y^{2}}
$$

This gives the final finite difference approximation of the 2D steady state heat equation for the point $(m, n)$ in the mesh as Equation (2.28). A similar equation can be derived for the three dimensional case.

$$
\frac{T_{m+1, n}-2 T_{m, n}+T_{m-1, n}}{\Delta x^{2}}+\frac{T_{m, n+1}-2 T_{m, n}+T_{m, n-1}}{\Delta y^{2}}+\frac{g}{\kappa}=0
$$

This process is repeated for every node in the mesh to get a unique linear equation linking temperatures and boundary conditions to the nodes around it. All these linear equations can be assembled into a matrix equation representing the set of $\mathrm{N}$ equations for $\mathrm{N}$ unknowns which for the steady-state case can be solved by matrix inversion.

\subsubsection{Rule of Mixtures}

When it came to meshing the large arrays of small vias in some oxide layers of the device structure, it was more efficient to minimize the amount of blocks in the mesh for these layers by approximating a material with an equivalent thermal conductivity using the rule of mixtures. While originally used to determine the effective resistivity of a multiphase solid, it is also useful in evaluating properties such as the effective dielectric constant, effective elastic modulus, and effective thermal conductivity [10, p. 139]. The equations for the rule of mixture and inverse rule of mixture models [33] are shown in Equations (2.29) and (2.30) where $\kappa_{\text {eff }}$ is the effective thermal conductivity in $\mathrm{W} / \mathrm{mK}$ in either the longitudinal or transverse direction, $\kappa$ is the thermal conductivity in the fiber or the matrix, and $\chi$ is the volume fraction of either the fiber or the matrix as calculated in Equation (2.31). The vias were defined to be the fiber and the oxide was defined to be the matrix, the transverse and longitudinal directions are dependant on which direction the heat is travelling through the structure and is 
shown in Figure 2.15.

$$
\begin{gathered}
\kappa_{e f f, l o n g}=\kappa_{f} \chi_{f}+\kappa_{m} \chi_{m} \\
\kappa_{\text {eff,trans }}=\left(\frac{\chi_{f}}{\kappa_{f}}+\frac{\chi_{m}}{\kappa_{m}}\right)^{-1} \\
\chi_{f}=\frac{V_{f}}{V_{t o t}}, \chi_{m}=\frac{V_{m}}{V_{t o t}}
\end{gathered}
$$
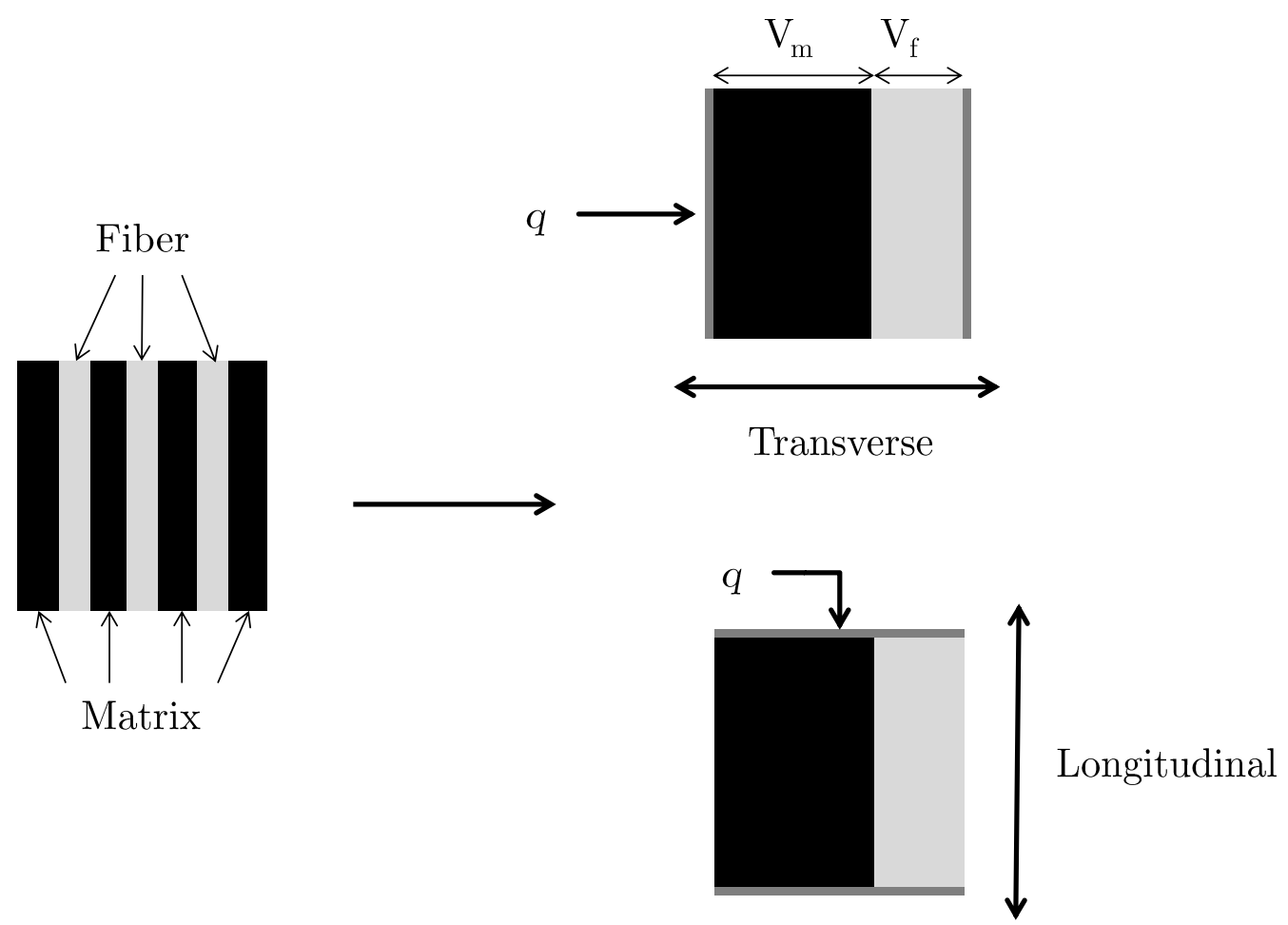

Figure 2.15: Visual representation of the fiber, matrix, volume fractions, and transverse/longitudinal directions for the rule of mixtures.

For these layers, there was an array of high thermal conductivity vias surrounded by a low thermal conductivity oxide, an is example shown in Figure 2.16. This would give a material with a directionally dependent thermal conductivity such that it would be much higher in the $z$ direction than it would be in the $x-y$ direction, though not 
zero. Using the rule of mixture and inverse rule of mixture models gives a good approximation for the material properties while lowering the mesh block count.
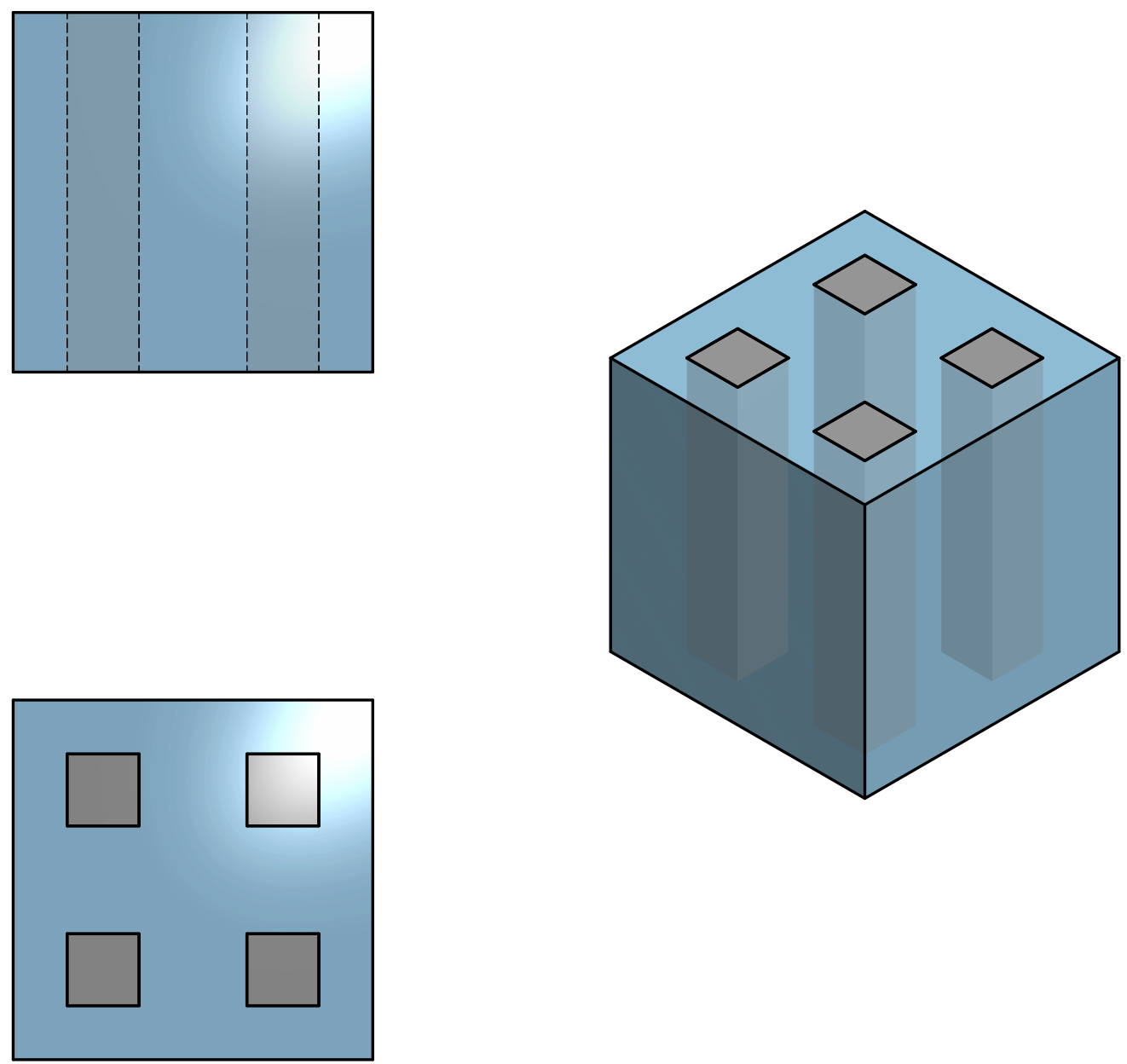

Figure 2.16: An example structure showing an array of high thermal conductivity via surrounded by low thermal conductivity oxide.

\subsubsection{Simulators}

The simulators that were considered for use through the course of this thesis were the commercial simulator COMSOL Multiphysics and the combination of Atar and OptiSPICE, simulators that were developed in-house at Carleton University. 


\section{COMSOL Multiphysics}

COMSOL Multiphysics is a suite of tools used for modelling and simulating engineering problems from a variety of domains including electromagnetics, structural mechanics, fluid flow and heat transfer, and chemical engineering. COMSOL has a variety of modules that can be linked to solve complex multiphysics problems, such as the Semiconductor module, the AC/DC module, and the Heat Transfer module. These three modules would be of most interest to the simulations involved in this thesis, and would allow for building accurate devices and modelling the electrothermal characteristics of the devices.

Investigation into the feasibility of the use of COMSOL for the simulations led to a few issues, primarily in the complexity of doing full 3D electrothermal simulations in both setup and performance. Device setup would have been difficult due to the lack of information on the exact device structure and properties (layout, doping, etc.) that would have been necessary to produce accurate simulations. Computer resources and simulation time were also an issue which led to the abandonment of COMSOL as an option for the simulations in this thesis.

\section{Atar and Optispice}

Atar is a 3D thermal simulation tool developed by Professor T.J. Smy at Carleton University [34]. Atar allows for the quick building and thermal simulation of a variety of integrated devices based on the technology and layout information provided by the user. It uses the finite difference method to solve the heat diffusion equation to establish the temperature distribution of the created device for both steady-state and transient problems.

For meshing the structures, Atar uses a rectangular discontinuous mesh based on an implementation of a 3D quad-tree meshing technique. The implementation of quad-tree meshing used by Atar creates a mesh of blocks of varying sizes such that 
each block can have a maximum of two connections on each vertical side and four connections each on the top and bottom, so each block can have a minimum of 6 to a maximum of 16 connections to surrounding blocks. An example for 2D and 3D quad-tree meshing is shown in Figure 2.17 [35]. This allows for a high-density mesh in areas with a high temperature gradient (such as around the heat sources) with a mesh that gets coarser as the temperature gradient gets smaller. The coarseness of the mesh can be controlled through masks defined in the technology file allowing for refinement of the meshing in areas determined to be of importance by the user. This meshing strategy allows Atar to generate an accurate solution while reducing simulation time.

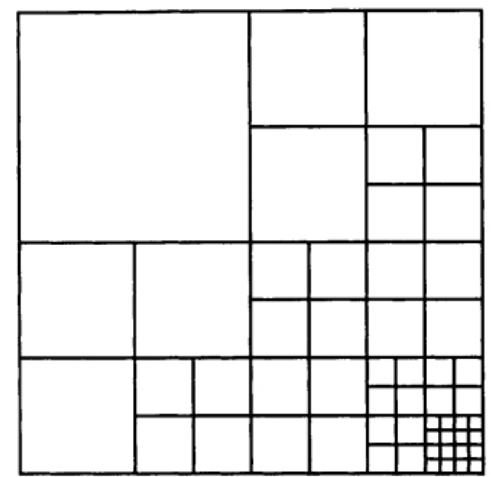

(a)

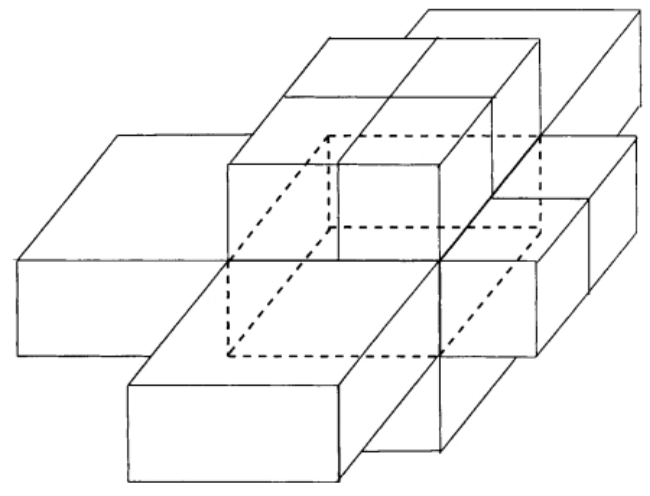

(b)

\section{Figure 2.17: Quad tree mesh example for (a) 2D mesh and (b) 3D mesh (used with permission from [35])}

Once the device is meshed, a thermal network is extracted consisting of resistors and capacitors. Each block in the model is visited and the thermal resistance to each neighbouring node is calculated using the common cross-sectional area, the size of the two blocks, and the local thermal conductivity [34]. The thermal capacitance of the material is used to calculate the capacitor connected from the block node to ground. Finally, boundary conditions are applied and the model is extracted for solution. Atar allows for direct computation of a solution using its internal solver, but also allows for the extraction of the model for use in an external solver such as OptiSPICE. 
Atar also implements algorithms for doing multi-dimensional model reduction (MDMR) for a steady state solution. This allows for large models to be reduced using a procedure, the mathematics of which are beyond the scope of this thesis but are shown in [36], to produce a reduced network that is very small compared to the original network. This allows for large networks to be reduced for more manageable simulation times while preserving the behaviour of the original system. Using the transformation produced by the MDMR reduction, the complete behaviour of the original system is able to be accurately obtained from the simulation results of the reduced system [36].

OptiSPICE is a MATLAB implementation of a commercial SPICE simulator, also developed by Professor Smy, that allows for easy linking of the thermal models produced by Atar into electrical simulations to allow for combined electrothermal simulations. This software was designed to work innately with Atar outputs through the use of general matrix elements that map the network of thermal resistances to a SPICE electrical netlist. OptiSPICE can then solve the thermal problem as an electrical equivalent with current representing power, and voltage representing temperature. 


\section{Chapter 3}

\section{Experiment Setup and Simulation Correlation}

\subsection{Simulation Setup}

\subsubsection{Simulator Selection}

Several simulators were investigated for this project but only two were given serious consideration. These simulators were COMSOL Multiphysics and Atar/OptiSPICE. Since the simulation was going to be a full three dimensional thermal simulation of the entire chip as shown in Figure 3.1, from the substrate up to the copper redistribution layer (RDL) including all the metallization and via patterns, performance of the simulator was a big concern. Issues arose when generating meshes in COMSOL for even a single cell as the scales involved in the structures that were built for this thesis (many micrometer sized features on a millimeter size chip) led to unreasonable processing requirements (central processing unit (CPU) and memory resources). Since the combination of Atar and Optispice were developed by Professor Smy at Carleton and it would be possible to adapt the program to suit the needs of this thesis with his help, Atar/OptiSPICE was chosen to build and simulate the devices used in this 
thesis.

\subsubsection{OptiSPICE Device from GaN Systems LTSpice Netlist}

The first step for setup of the simulators was to adapt the Spice model files provided by GaN Systems for use in OptiSPICE. The LTSpice models available on GaN Systems' website are for the full device but what was needed for this experiment was the model of an individual island transistor. GaN Systems provided the model file which followed the same circuit structure as the full chip as shown in Figure 3.2 but had some equations altered to make it scalable according to the gate width $\left(W_{g}\right)$. All the individual islands are connected in parallel to provide the equivalent of a larger transistor with combined $W_{g}$ of all active islands while providing a measure of fault tolerance [6]. The individual cell model cannot be provided as it's covered under a non-disclosure agreement (NDA) but the full device model file is included in Appendix A.1 for reference.

In order to bring the model into OptiSPICE, the derivatives with respect to the voltages in the equations of all the arbitrary current sources and voltage dependent capacitors were needed; the list of which are provided in Table 3.1. The mathematical software Maple was used to provide the derivation to avoid any calculation or transcription errors as it was able to symbolically calculate the derivatives and provide Matlab formatted outputs directly.

Once the derivatives were calculated, the formulas for the arbitrary current sources and voltage-dependent capacitors were all written into Matlab functions in a single file to become the Update file for all the currents and capacitor charges of the islands for the Spice solution. The Update file was then provided to Professor Smy and a custom general electrical device was created for OptiSPICE that represents an individual island cell with four inputs: Gate, Drain, Source, and T1. The T1 port is used as an input for the junction temperature, which will enable the modelling of 


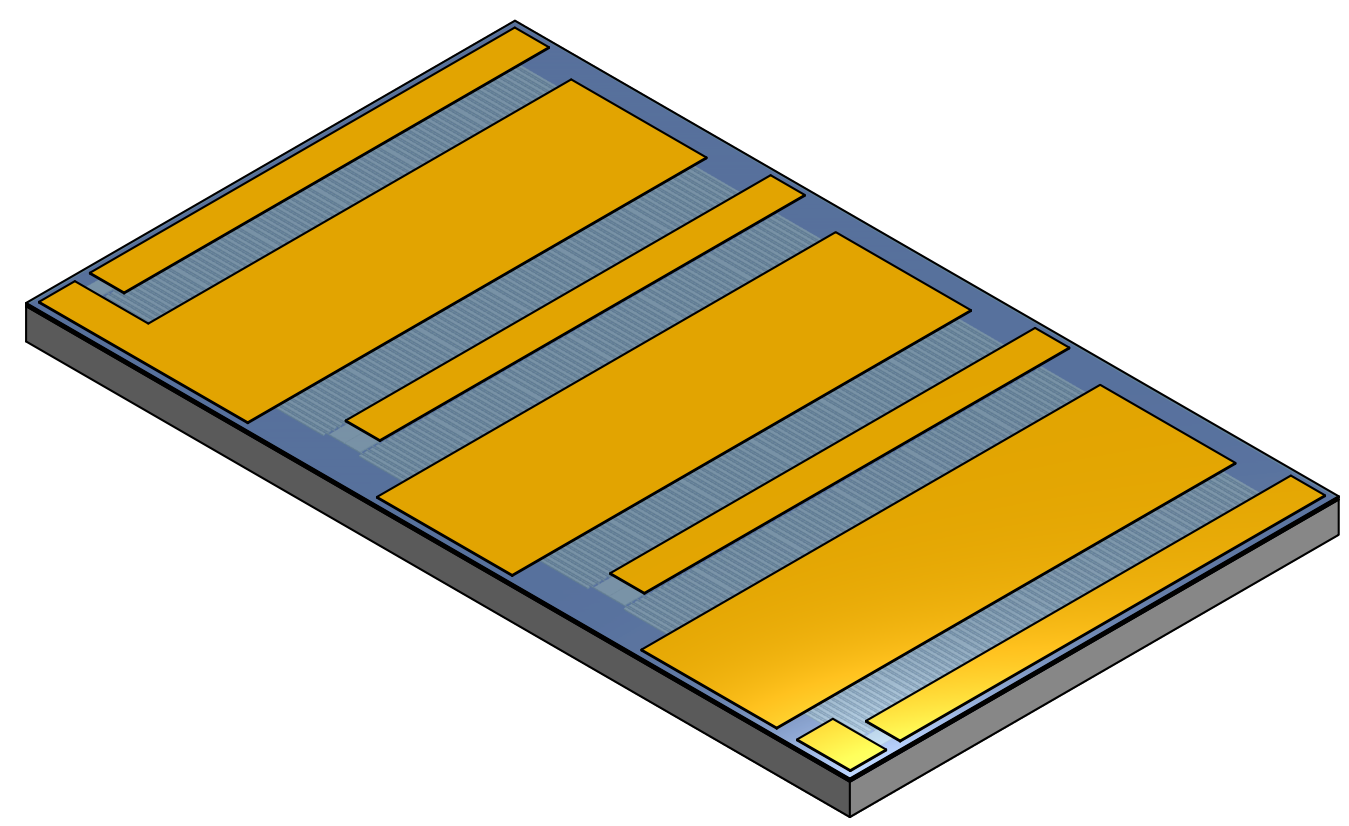

(a) 3D computer-aided design (CAD) model of the chip structure showing all layers from the substrate up to the copper RDL.

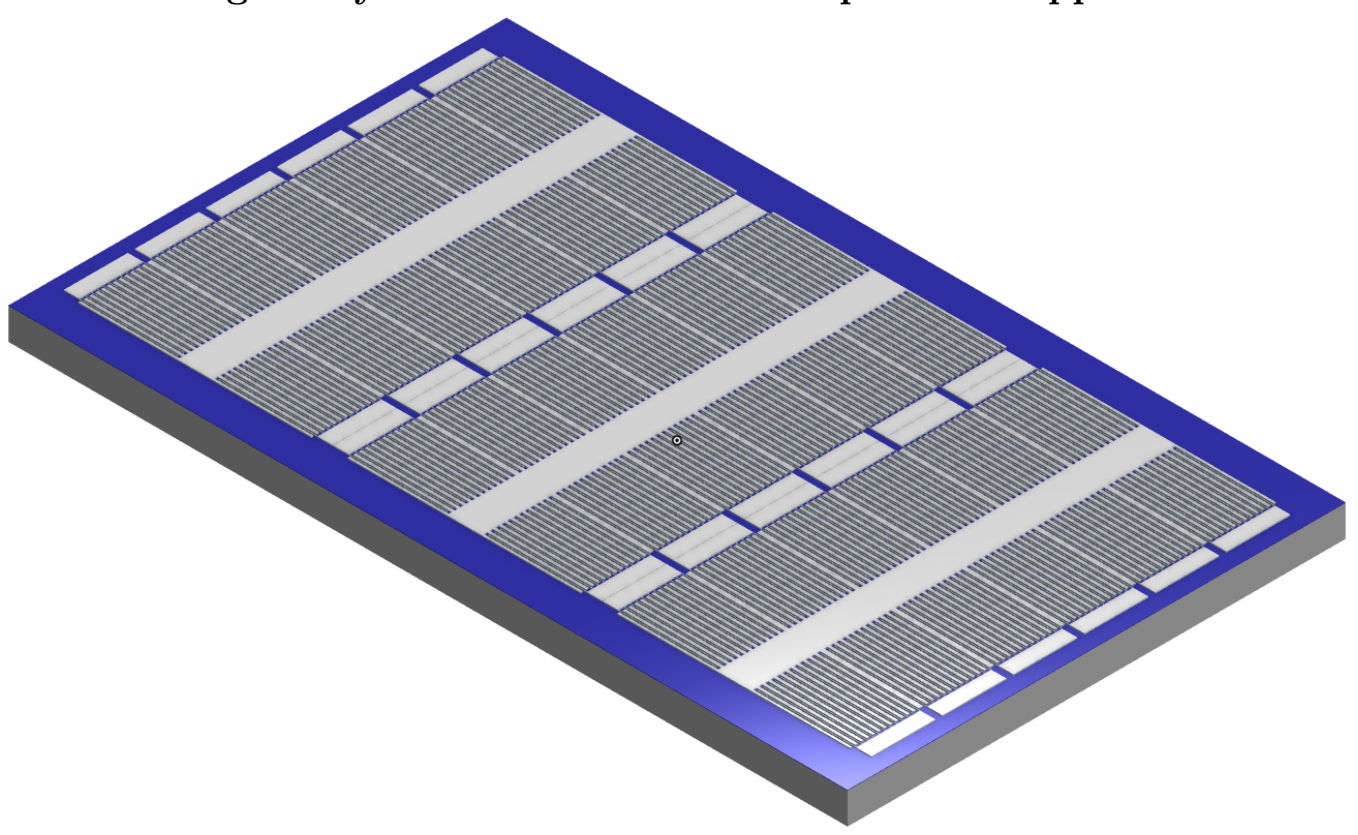

(b) 3D CAD model of the chip structure without upper layers to show cell layout.

Figure 3.1: 3D CAD representation of the chip structure to be simulated. 

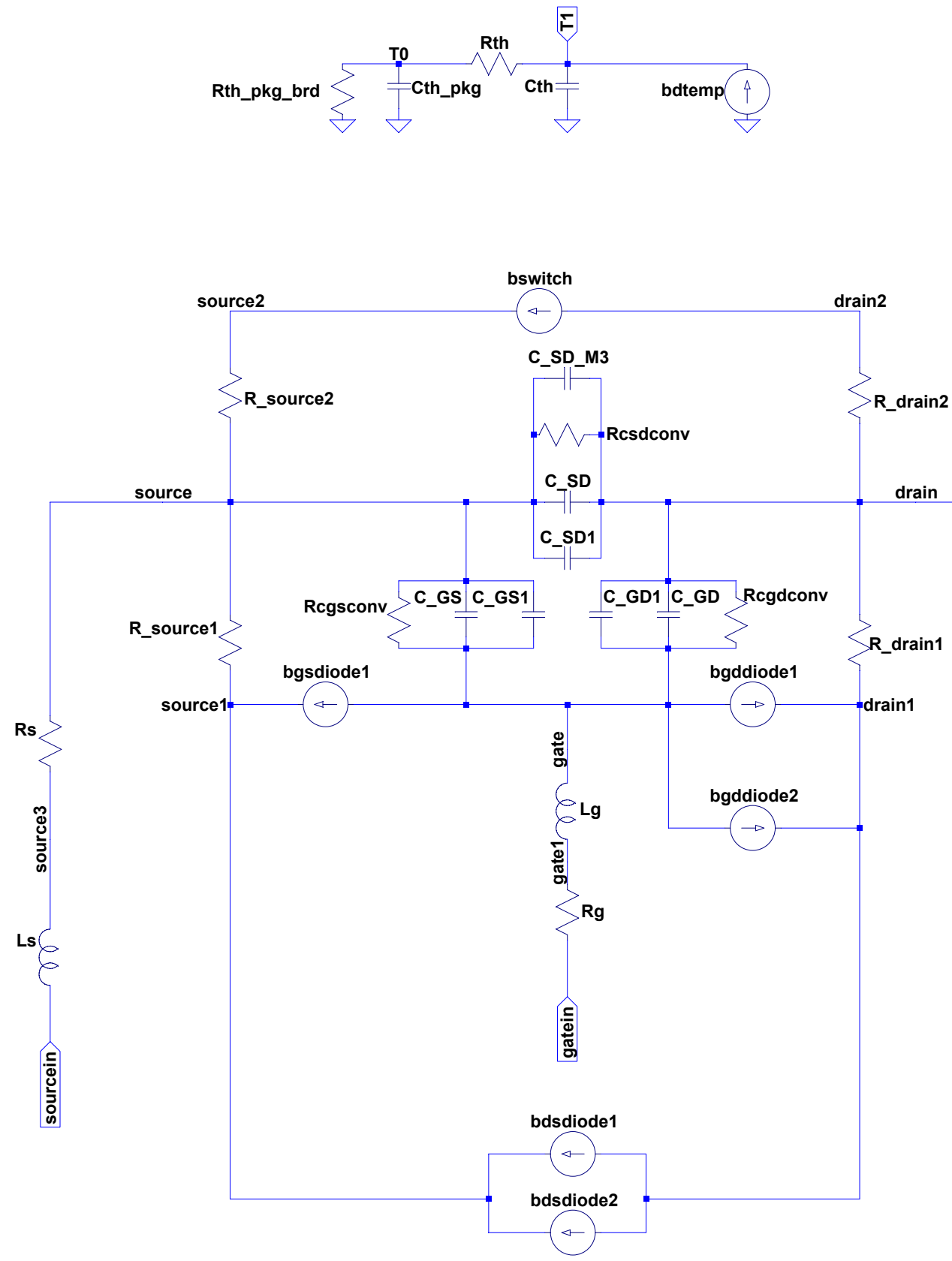

Ld

Figure 3.2: SPICE circuit built from GS66516T model netlist (example netlist shown in Appendix A.1). 
Table 3.1: List of derivatives for arbitrary current sources and voltage-dependent capacitors.

\begin{tabular}{|c|c|c|}
\hline Circuit Element Type & Name & Derivatives \\
\hline \multirow{7}{*}{ Arbitrary Current Sources } & $B_{d t e m p}$ & $\frac{d B_{d t e m p}}{d V_{T 1}}, \frac{d B_{d t e m p}}{d V_{g}}, \frac{d B_{d t e m p}}{d V_{s}}$ \\
\hline & $B_{\text {switch }}$ & $\frac{d B_{\text {switch }}}{d V_{T 1}}, \frac{d B_{\text {switch }}}{d V_{g}}, \frac{d B_{\text {switch }}}{d V_{s 2}}, \frac{d B_{\text {switch }}}{d V_{d 2}}$ \\
\hline & $B_{G S d i o d e 1}$ & $\frac{d B_{G S \text { diode } 1}}{d V_{g}}, \frac{d B_{G S \text { diode } 1}}{d V_{s 1}}$ \\
\hline & $B_{G D d i o d e 1}$ & $\frac{d B_{G D d i o d e 1}}{d V_{g}}, \frac{d B_{G D d i o d e 1}}{d V_{d 1}}$ \\
\hline & $B_{G D \text { diode } 2}$ & $\frac{d B_{G D d i o d e 2}}{d V_{g}}, \frac{d B_{G D d i o d e 2}}{d V_{d 1}}$ \\
\hline & $B_{D S d i o d e 1}$ & $\frac{d B_{D S \text { diode1 } 1}}{d V_{d 1}}, \frac{d B_{D S \text { diode } 1}}{d V_{s 1}}$ \\
\hline & $B_{D S d i o d e 2}$ & $\frac{d B_{D \text { Sdiode } 2}}{d V_{d}}, \frac{d B_{D \text { Sdiode } 2}}{d V_{s}}$ \\
\hline \multirow{3}{*}{ Voltage-Dependent Capacitors } & $C_{S D 1}$ & $\frac{d C_{S D 1}}{d V_{s}}, \frac{d C_{S D 1}}{d V_{d}}$ \\
\hline & $C_{G S 1}$ & $\frac{d C_{G S 1}}{d V_{g}}, \frac{d C_{G S 1}}{d V_{s}}, \frac{d C_{G S 1}}{d V_{d}}$ \\
\hline & $C_{G D 1}$ & $\frac{d C_{G D 1}}{d V_{g}}, \frac{d C_{G D 1}}{d V_{d}}$ \\
\hline
\end{tabular}

the electrothermal characteristics of the device once the thermal model is created and linked to the electrical model.

\subsubsection{Device Structure Setup for Atar}

The thermal simulation program Atar was originally written for Mac OSX and was required to be ported over to Linux for use on the Carleton University Department of Electronics servers to take advantage of the increased processing power and memory resources. While porting the program to Linux, alterations were made with the help of Professor Smy to adapt the program to the needs of the structures for this thesis including increasing the block count, the ability to individually control the generation regions, and general stability fixes when working with the large structures produced.

Two different structures were created for thermal simulation in Atar. The first device simulated was a simple model used to determine whether the results that Atar 
provided matched a known quantity, in this case a thermal simulation previously performed by GaN Systems using a commercial thermal simulator. The second device was an approximate representation of the full device structure from the substrate up to the copper RDL.

\section{Thermal Simulation Correlation Structure}

To correlate the Atar simulations with a known thermal simulation, the structure being simulated had to match that used in the previously performed thermal simulation. In this case, it was a simple structure composed of a thick silicon substrate with a thin GaN layer on top with 36 regions in the GaN layer defined as generating regions. The structure is shown in Figure 3.3, with the grey representing the silicon substrate, the blue representing the GaN layer and the red showing the generating regions. In the Atar output in Figure 3.3b, only 6 of the generating regions on left side are shown. The other 30 regions do not appear in this visualization due to the tiling method used. Due to the top face of the generation region and the top face of the GaN layer coinciding, there are slight visual glitches in the Atar output. This visual glitch will appear in any Atar output where the generation region appears.

\section{Full Device Structure}

The full chip structure was built using information provided by GaN Systems of an approximate representation of the device layout and material stackup. One approximation was to the $\mathrm{AlGaN} / \mathrm{GaN}$ heterostructure which was treated as a single $\mathrm{GaN}$ layer. Although $\mathrm{AlGaN}$ has been shown to have a lower thermal conductivity than GaN [37], the AlGaN layer is much thinner than the GaN layer so this approximation shouldn't have a significant effect on the thermal distribution. Another approximation occurred in the shape of the metal layers. In the actual device layout, rectangular 


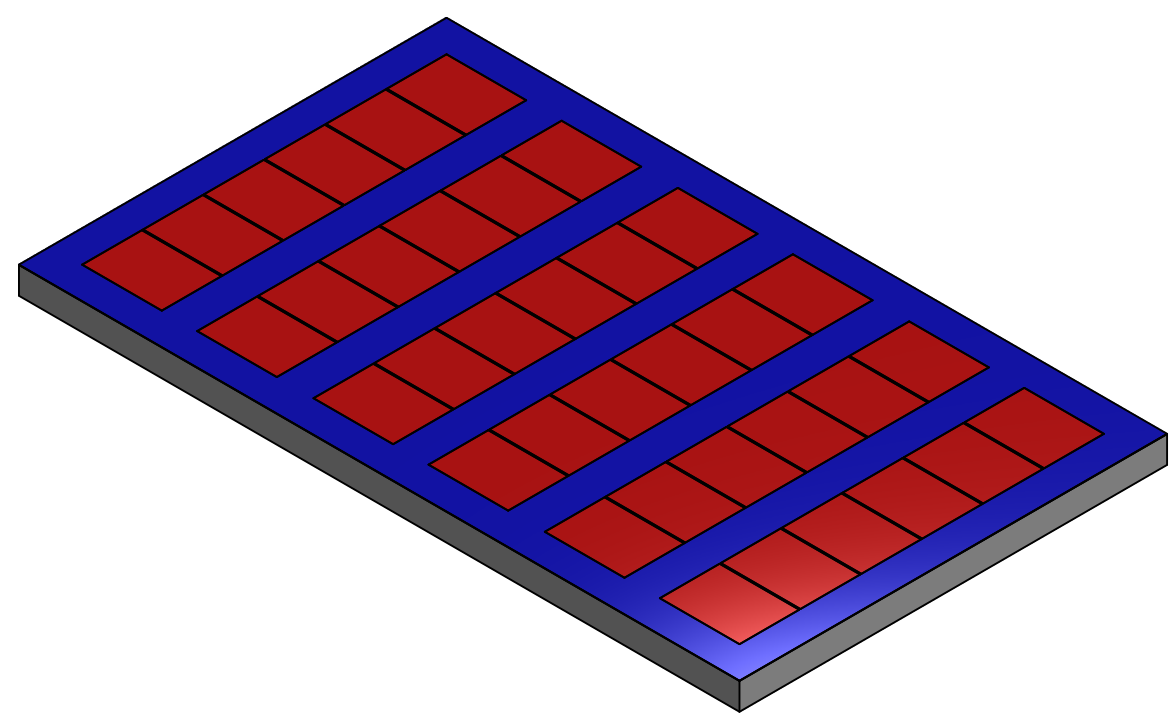

(a) 3D CAD model of the thermal simulation correlation structure showing the silicon substrate (grey), GaN layer (blue), and generating regions (red).

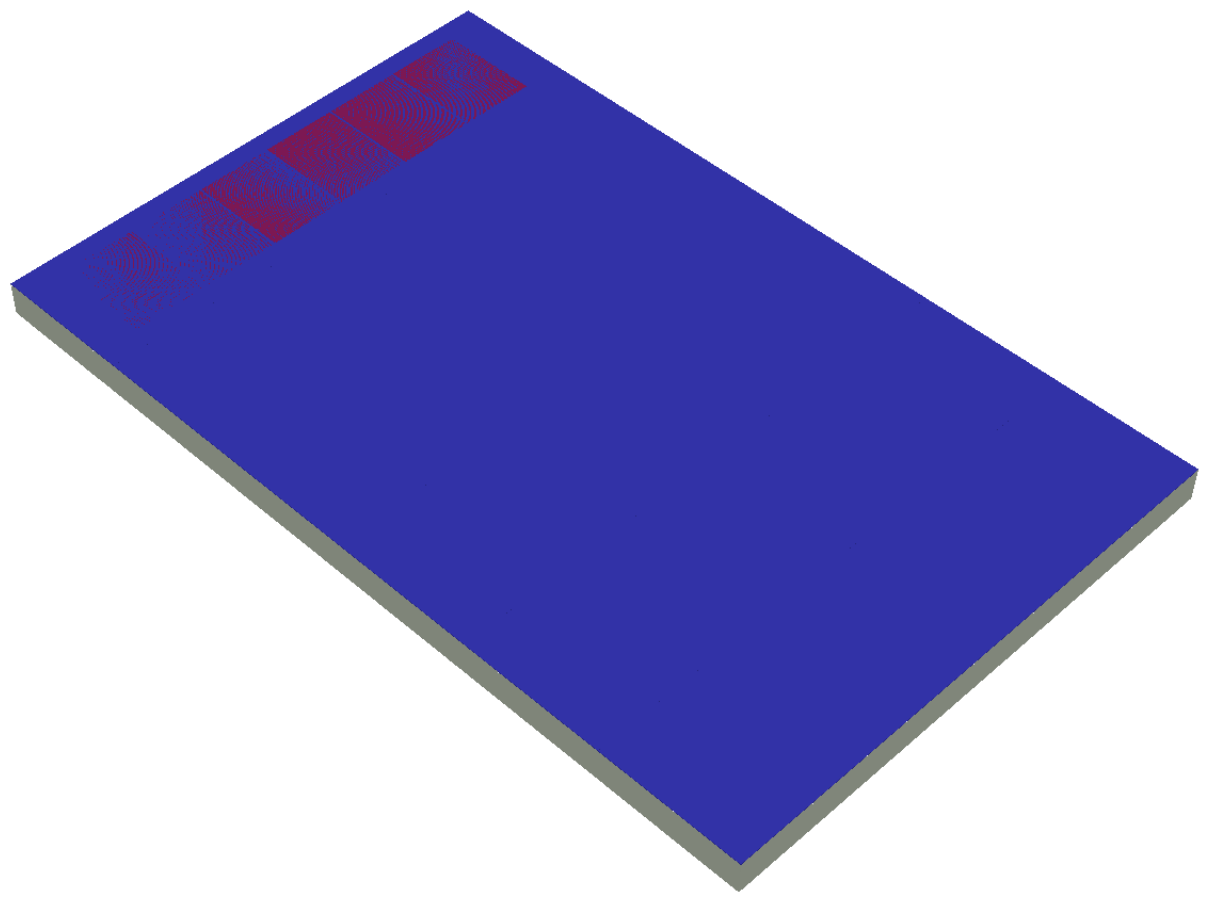

(b) Atar output of the thermal simulation correlation structure showing only six generating regions on the left hand side.

Figure 3.3: 3D CAD model of the thermal correlation structure and Atar output of the same structure. (Note: The visual glitches seen in Figure $3.3 \mathrm{~b}$ are due to the coinciding generation region and $\mathrm{GaN}$ surfaces) 
metal elements would be avoided due to the crowding of electric fields at sharp electrode corners which could lead to premature breakdown [38]. Though the metal layers provided were rectangular rather than rounded, this should have minimal effect on the thermal behaviour and works well with the rectangular meshing capabilities of Atar. The information on the exact dimensions and materials used is proprietary to GaN Systems and has been left out of the thesis due to the NDA signed before beginning the project.

The cell starts with a layer of GaN with a thin generating region underneath the source and drain fingers to represent the power dissipated by the 2DEG. As was the case with the thermal correlation structure presented in Figure 3.3b, the generating region face coincides with the GaN layer causing the visual bug seen in this layer (Figure 3.4a). Next, the vias to the source and drain fingers were created using a material with an effective thermal conductivity for a slab of vias surrounded by oxide. The effective medium thermal conductivity in the longitudinal and transverse directions was determined using the rule of mixtures method as presented in Section 2.4.3. The effective thermal conductivity was higher in the longitudinal direction $(44.3 \mathrm{~W} / \mathrm{mK})$ than it was in the transverse direction $(1.86 \mathrm{~W} / \mathrm{mK})$. Only the effective medium appears (shown in green) as oxide is invisible in Atar (Figure 3.4b). The next layer is Metal 1 (M1), which is the source and drain fingers as shown in light blue (Figure 3.4c). Following this is the via layer to connect M1 to M2, again the vias in oxide were represented by an effective medium. The via region existed under the fingers as well as the finger connector metal so the via layer was divided into multiple different regions to determine individual effective thermal conductivities for the regions depending on the size of the region and density of vias. These different materials are shown in bright green, dark green, red, and brown (Figure 3.4d). Next is the M2 layer of source and drain fingers, slightly thicker than the M1 layer, shown in light blue (Figure 3.4e). On top of M2 is a layer of passivation in blue-green, with 
contacts from the copper RDL to connect to M2 in yellow (Figure 3.4f). Next is the isolation layer shown in purple, again with contacts from the copper RDL to connect to M2 in yellow (Figure 3.4g). The final layer of the structure is the copper RDL, used as a bus to connect the drain and source of each transistor in parallel (Figure 3.4h). A summary of the layout information is included in Table 3.2.

Table 3.2: Summary of the Atar single cell full structure layout (without substrate).

\begin{tabular}{|c|c|c|c|}
\hline Layer & Material & Colours & Purpose \\
\hline $\mathrm{a}-\mathrm{GaN}$ & Gallium nitride & Dark blue & $\begin{array}{l}\text { Active layer, contains } \\
\text { generating region }\end{array}$ \\
\hline b-GaN to M1 Via & $\begin{array}{l}\text { Effective via } \\
\text { medium } 1\end{array}$ & Green & $\begin{array}{l}\text { Connects drain and } \\
\text { source to active GaN } \\
\text { layer }\end{array}$ \\
\hline c-M1 & Aluminium & Light blue & $\begin{array}{l}\text { Drain and source } \\
\text { fingers }\end{array}$ \\
\hline d - M1 to M2 Via & $\begin{array}{l}\text { Effective via } \\
\text { medium } 2 \text { to } 5\end{array}$ & $\begin{array}{l}\text { Bright green, } \\
\text { dark green, } \\
\text { red, and } \\
\text { brown }\end{array}$ & Connects M1 to M2 \\
\hline e - M2 & Aluminium & Light blue & $\begin{array}{l}\text { Thicker metal for } \\
\text { enhanced current } \\
\text { handling }\end{array}$ \\
\hline f - Passivation & $\begin{array}{l}\text { Silicon nitride } \\
\text { and copper }\end{array}$ & $\begin{array}{l}\text { Blue-green } \\
\text { and yellow }\end{array}$ & $\begin{array}{l}\text { Passivation of device } \\
\text { surface }\end{array}$ \\
\hline g - Isolation & $\begin{array}{l}\text { Polyimide and } \\
\text { copper }\end{array}$ & $\begin{array}{l}\text { Purple and } \\
\text { yellow }\end{array}$ & Isolation of devices \\
\hline h - Copper RDL & Copper & Yellow & $\begin{array}{l}\text { Connecting source } \\
\text { and drain of each } \\
\text { island into parallel } \\
\text { bus }\end{array}$ \\
\hline
\end{tabular}

The device was built starting from a single cell as shown in Figure 3.4 set on a thick silicon substrate. This cell was mirrored to form a pair of cells that share a source connection as shown in Figure 3.5. This mirrored structure was then tiled to achieve 
an array of 6 by 6 individual island transistors. The full array of island transistors up to the M2 layer is shown in Figure 3.6 to illustrate the device layout. Each individual island transistor has its own controllable generation region that can be assigned a power either in Atar directly for pure thermal simulations or in OptiSPICE through the thermal models produced by Atar for the linked electrothermal simulations. The addressable cells were given the numbering scheme as shown in Figure 3.7 and this numbering scheme will be referenced in the simulations when discussing which cells have been deactivated.

The final Atar chip structure contained 25.5 million blocks, with each block containing a node with a connection to all surrounding nodes. Each node corresponds to a unique linear equation linking the temperatures and boundary conditions to the nodes around it but also to every other node in the structure. Since most of the 25.5 million nodes are not connected to each other, this leads to the matrix of equations being a sparse matrix which contains mostly zeros. The $\mathbf{G}$ thermal conduction matrix for the final Atar structure was a 6.6 gigabyte 25.5 million by 25.5 million sparse matrix of thermal conductances. Reducing this system using the MDMR algorithms built into Atar required over 6 hours to complete and used over 280 gigabytes of memory on the servers. The result of the MDMR was a 21.6 gigabyte MATLAB .mat file that contained both the full system along with the reduced system information for use in the electrothermal simulations in OptiSPICE. The reduced G matrix is only 43 by 43 and the transformation matrix that maps the reduced system back to the original system is 25.5 million by 43 . 


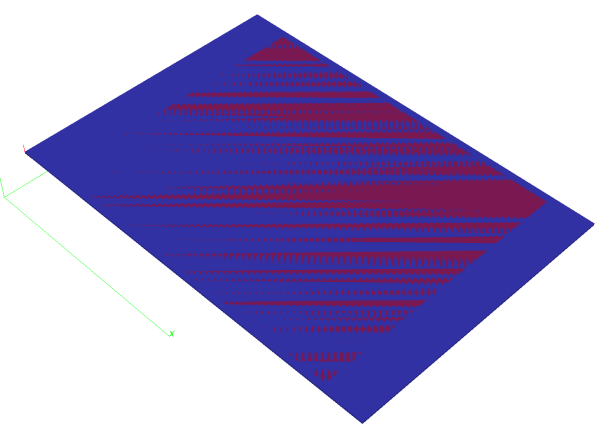

(a) GaN Layer

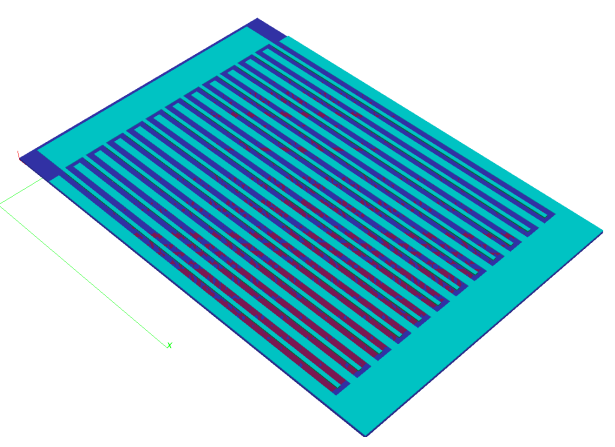

(c) M1 Layer

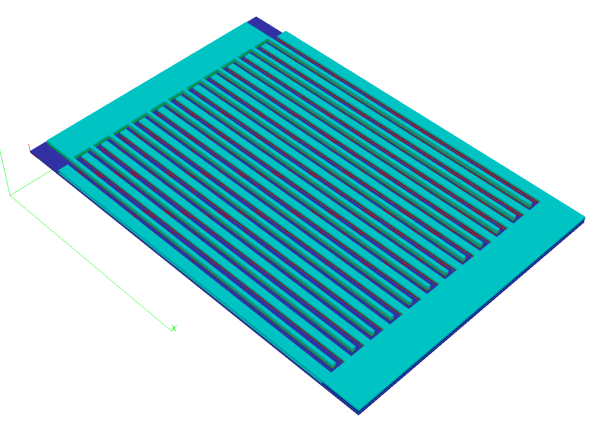

(e) M2 Layer

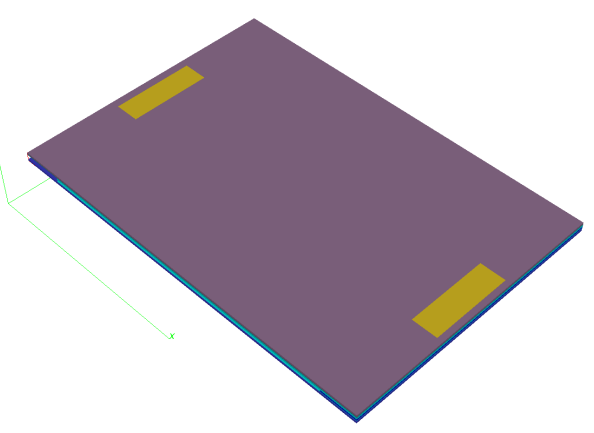

(g) Isolation Layer

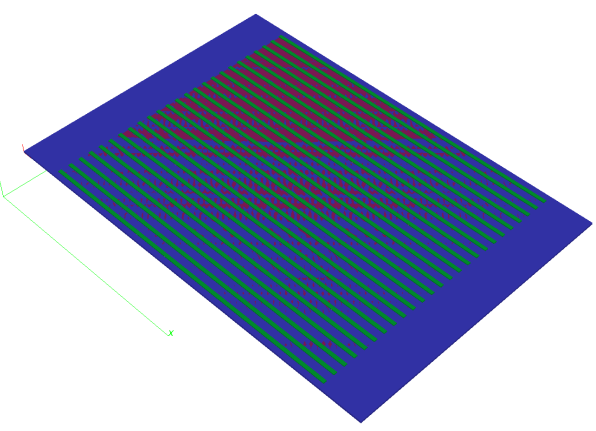

(b) GaN to M1 Via Layer

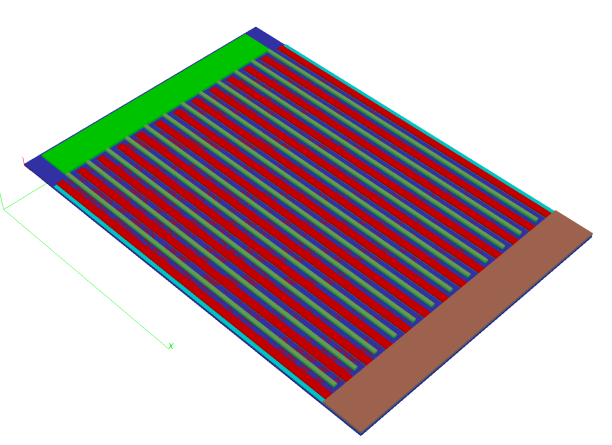

(d) M1 to M2 Via Layer

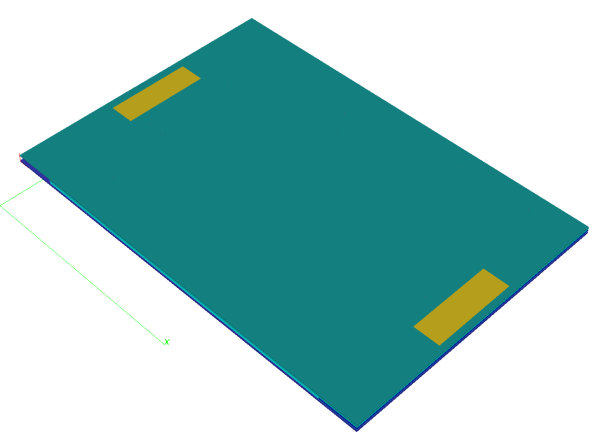

(f) Passivation Layer

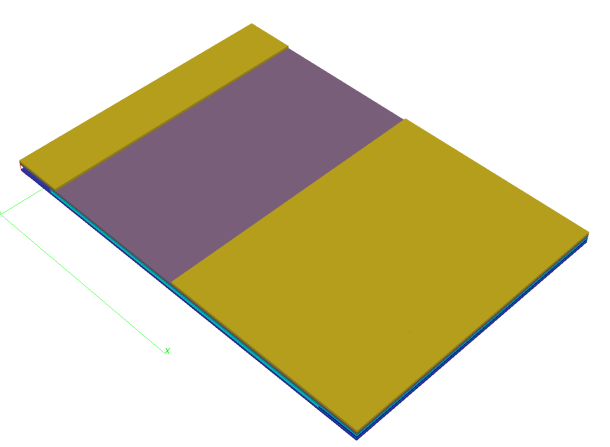

(h) Copper RDL Layer

Figure 3.4: Layers of the single cell stack-up built in Atar. (Note: The visual glitch seen in Figure 3.4a/3.4b is due to the coinciding generation region and GaN surfaces) 


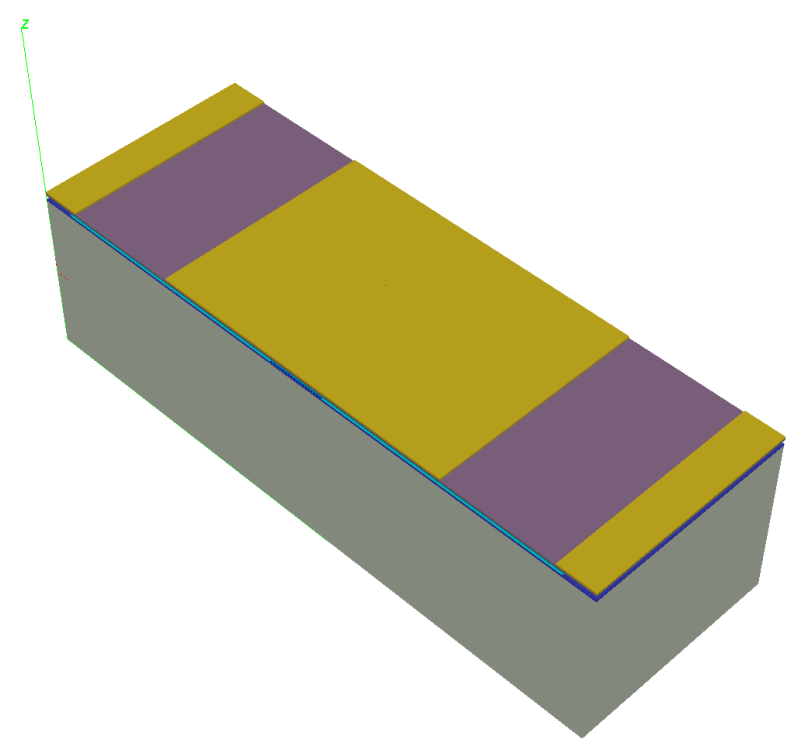

Figure 3.5: A mirrored cell structure showing a pair of cells that share a source connection.

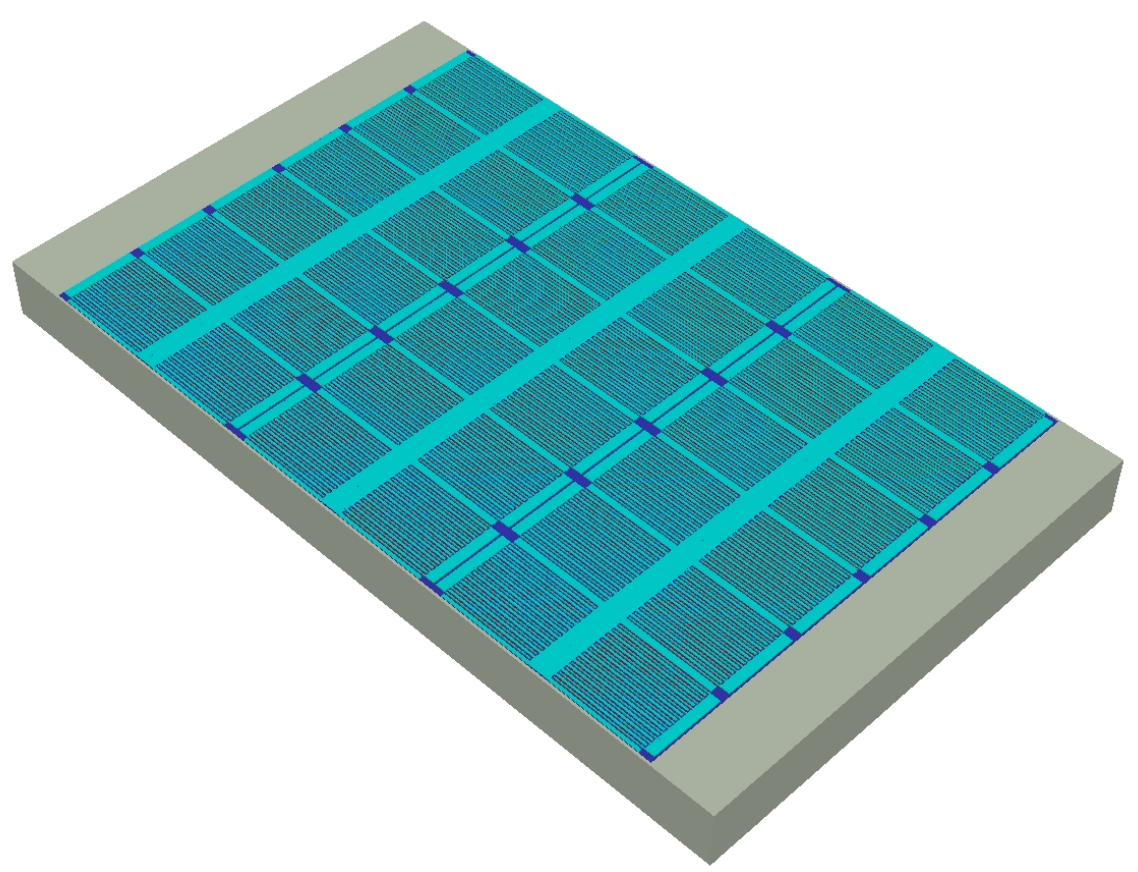

Figure 3.6: The full chip structure up to the M2 layer showing the 36 individual cells. 


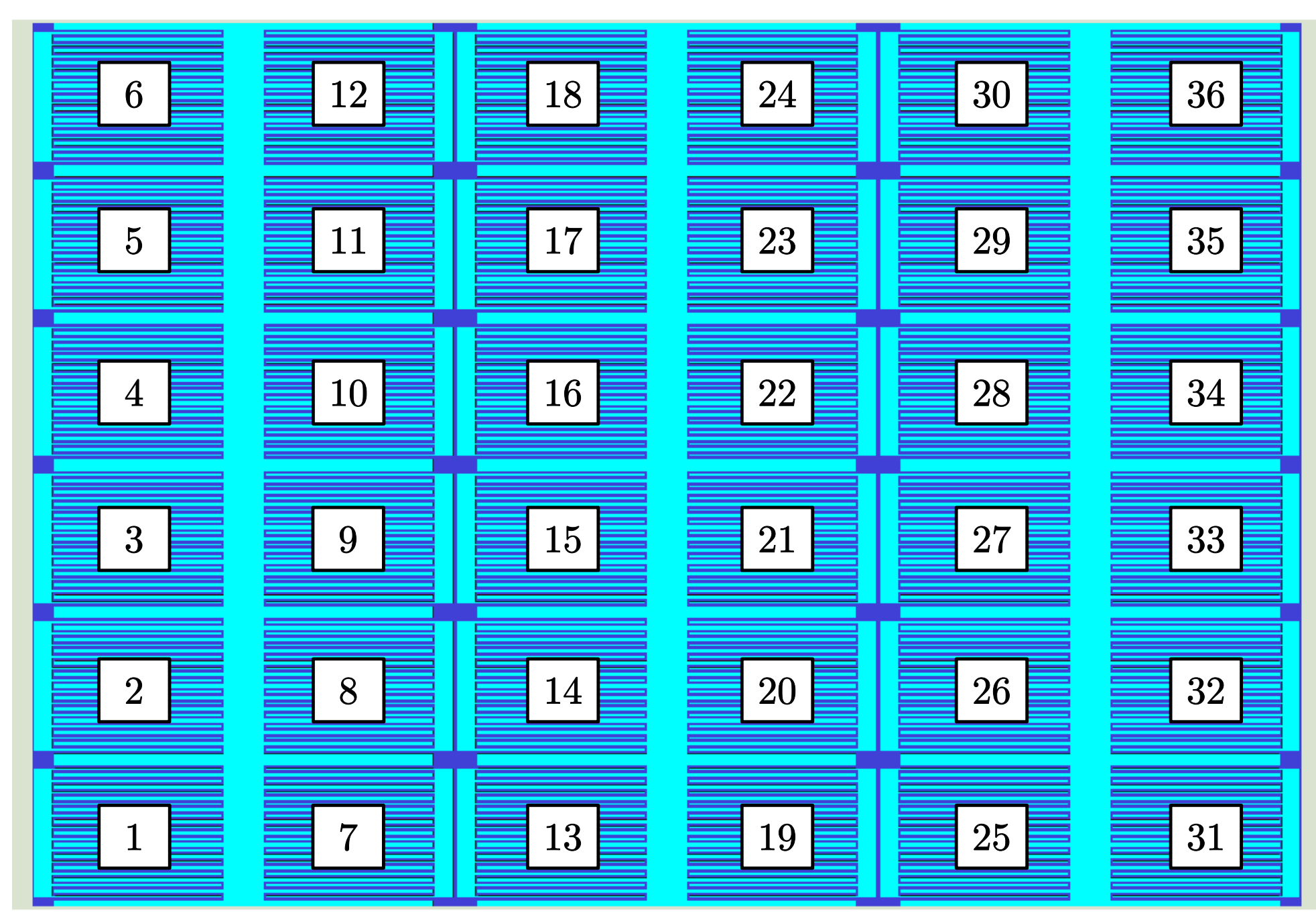

Figure 3.7: Number map of the 36 cells in the full chip structure used for reference throughout this thesis. 


\subsubsection{Electrothermal Setup for OptiSPICE}

The reduced thermal models produced by Atar were linked into OptiSPICE through the use of a general matrix model element. This matrix element has 36 ports to which current is applied, which corresponds to a power applied to each cell generation region. A voltage is then generated on this same port, which corresponds to the temperature of the generation region. The applied power is introduced into the model by splitting it between all the blocks that define the cell generation region based on the volume of each block assuming constant generation within the region. Each block in the generation region will then have a unique temperature and the voltage output is calculated as a volume averaged value of each temperature for the generation region.

In the OptiSPICE electrothermal netlist, each cell in the array consists of the elements detailed in Table 3.3 and illustrated in Figure 3.8. Each of the cells in the array are connected to the common drain and gate voltages and have their sources grounded. To deactivate an individual cell, the gate connection and drain connection to the cell are left 'floating', disconnecting the transistor from the array. Since the cell is disconnected from the array, no power goes to the matrix element which means no generation occurs in that cell for the thermal distribution but the generation region still has a temperature due to the backside temperature and the thermal gradient of the cells surrounding it. This means that a disconnected cell inputs no power into the thermal model matrix but the temperature of the cell is still calculated and given as an output. OptiSPICE solves the electrothermal problem by iteratively solving the electrical and thermal circuit until the result converges for an operating point. An example input file for OptiSPICE is included for reference in Appendix B.1. Each individual electrothermal simulation takes roughly 3 minutes to run which is only possible due to the reduced system produced by Atar using MDMR, otherwise the electrothermal simulations would take too long to complete making multiple simulation runs for different operating conditions (voltages and cell distributions) infeasible 
or entirely impossible.

Table 3.3: Summary of OptiSPICE electrothermal netlist elements

\begin{tabular}{|c||l|l|l|}
\hline Element & Purpose & Inputs & Outputs \\
\hline $\mathbf{X} \boldsymbol{n}$ & $\begin{array}{l}\text { Island Transistor Model for } \\
\text { Cell }\end{array}$ & vGate, VD $n, \mathrm{~T} 1 n$ & $\mathrm{X} n: \mathrm{I}$ \\
\hline M1:Cn & Thermal Model for Cell & $\mathrm{C} n: \mathrm{I}$ & $\mathrm{C} n: \mathrm{V}$ \\
\hline Pow $\boldsymbol{n}$ & $\begin{array}{l}\text { Calculates power from cell } \\
\text { drain voltage and current }\end{array}$ & VSEN $n: \mathrm{I}, \mathrm{VD} n$ & $\begin{array}{l}\text { Pow } n: \mathrm{I} \\
\mathrm{VD} n * \mathrm{VSEN} n: \mathrm{I}\end{array}$ \\
\hline VSEN $\boldsymbol{n}$ & $\begin{array}{l}\text { Zero voltage source to ob- } \\
\text { tain drain current for calcu- } \\
\text { lating power }\end{array}$ & $\mathrm{VDD}$ & $\mathrm{VD} n=\mathrm{VDD}$ \\
\hline Temp $\boldsymbol{n}$ & $\begin{array}{l}\text { Voltage controlled voltage } \\
\text { source to input junction } \\
\text { temperature as voltage onto } \\
\text { T1 port to prevent loading } \\
\text { from the matrix element }\end{array}$ & $\mathrm{C} n: \mathrm{V}$ & $\mathrm{T} 1 n: \mathrm{V}=\mathrm{C} n: \mathrm{V}$ \\
\hline
\end{tabular}

\subsection{Simulator Correlation}

\subsubsection{OptiSPICE Electrical Simulation Correlation}

To verify that the LTSpice models were ported to OptiSPICE correctly, electrical simulations were run in both LTSpice and OptiSPICE using the same parameters. The LTSpice simulations used the full device model posted to GaN Systems' website (also included in Appendix A.1) while the OptiSPICE simulations used the island transistor models connected in a parallel array. The parameters were chosen to match the $\mathrm{I}_{\mathrm{DS}}$ vs. $\mathrm{V}_{\mathrm{DS}}$ characteristics from the GS66516T datasheet [7], sweeping $\mathrm{V}_{\mathrm{DS}}$ from $0 \mathrm{~V}$ to $5 \mathrm{~V}$ for $\mathrm{V}_{\mathrm{GS}}$ values of $2 \mathrm{~V}$ to $6 \mathrm{~V}$ in $1 \mathrm{~V}$ steps. The output of each simulator is compared to the data extracted from the datasheet in Figure 3.9. The $I_{D S}$ vs. $V_{D S}$ curves for the OptiSPICE parallel array simulation lie between the datasheet extracted values and the LTSpice full device model simulation. The input file for the OptiSPICE 


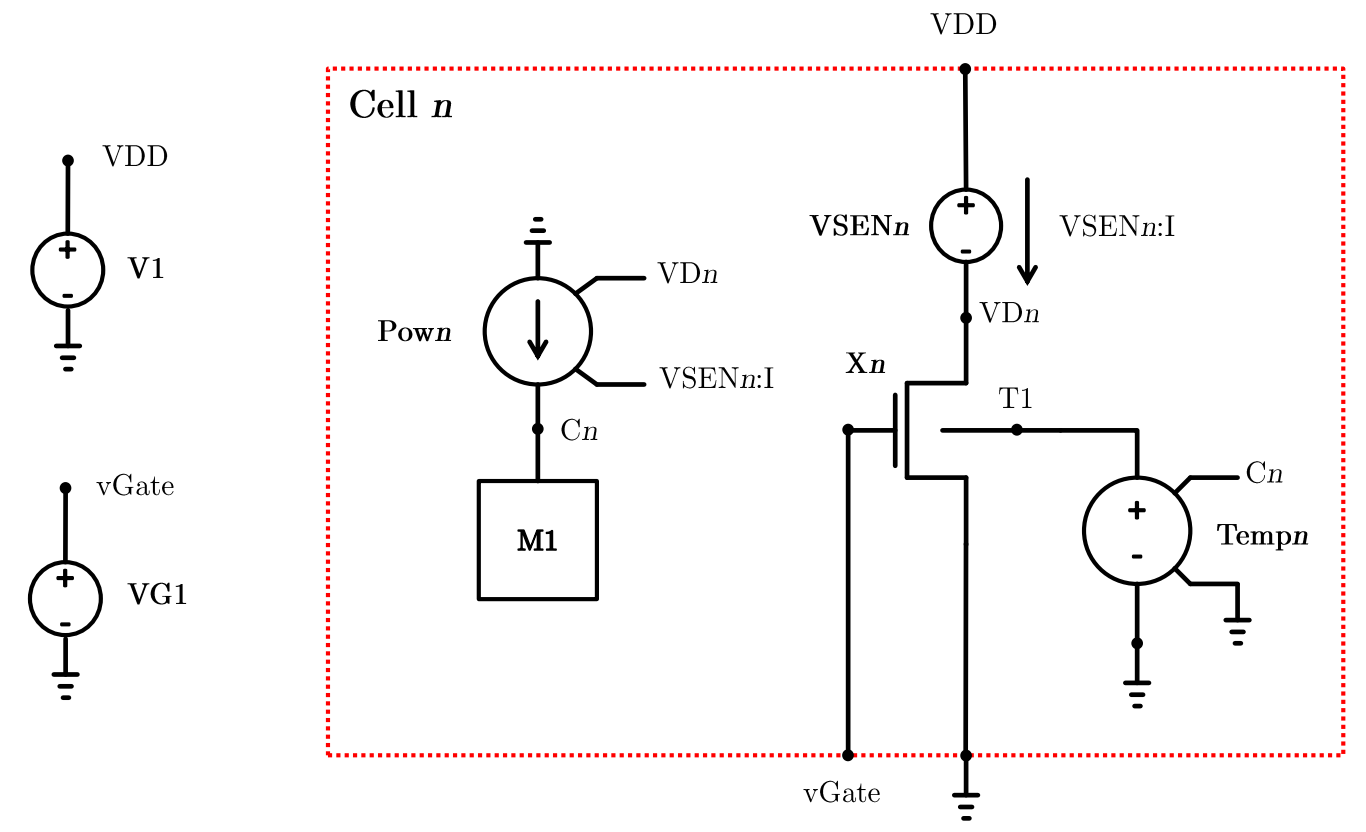

Figure 3.8: OptiSPICE electrothermal SPICE circuit of common sources (left), and of an individual cell (right, red box).

electrical simulation is included for reference in Appendix B.2. 
$\mathrm{I}_{\mathrm{D}}$ vs. $\mathrm{V}_{\mathrm{DS}}$ Comparison Between Datasheet, LTSpice Full Model,

and OptiSPICE Parallel Array Model

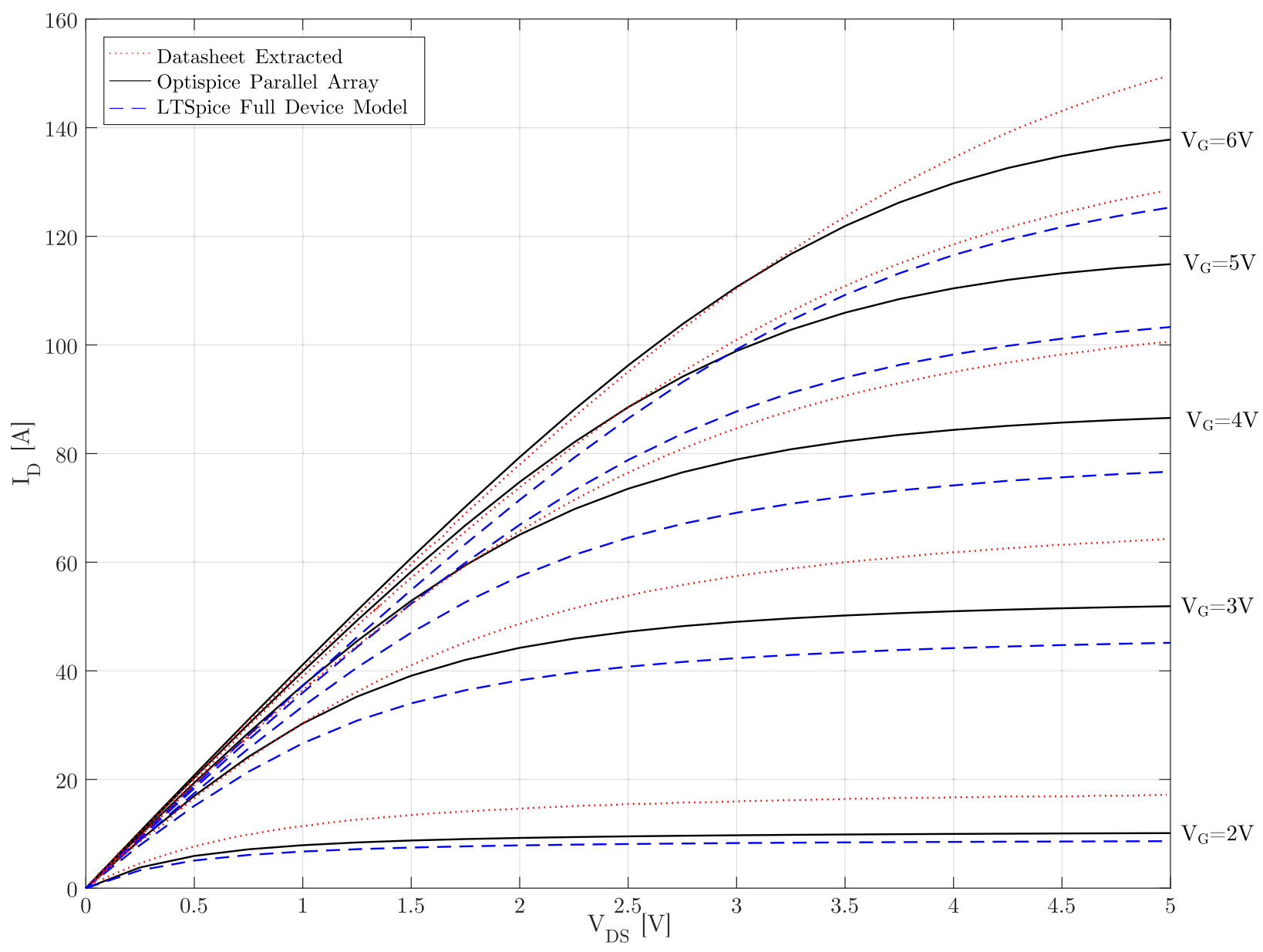

Figure 3.9: $I_{D S}$ vs $V_{D S}$ curves comparing the electrical outputs of the GS66516T datasheet, the LTSpice simulation using the GaN Systems' full device model, and the OptiSPICE simulation using island transistors in a parallel array. 


\subsubsection{Atar Thermal Simulation Correlation}

To verify that Atar would be accurate for simulating the correct thermal behaviour for the final electrothermal simulations, it was necessary to compare the results of a simple Atar simulation to a known simulation result produced by GaN Systems. Simulating the simplified geometry correlation structure introduced in Section 3.1.3 for $100 \mathrm{~W}$ of power evenly distributed amongst the 36 cells with a fixed backside temperature of $25^{\circ} \mathrm{C}$, the results shown in Figure 3.10 were obtained. A maximum temperature of $42.30^{\circ} \mathrm{C}$ was achieved (Atar output included in Appendix C.1) compared to the $42.14^{\circ} \mathrm{C}$ that the GaN Systems thermal simulation obtained as seen in Figure 3.11. This difference of $0.16^{\circ} \mathrm{C}(0.4 \%$ error $)$ was small enough to consider Atar to be equivalent to the commercial software in use by GaN Systems and therefore suitable for simulating the thermal aspects of the device.

\subsubsection{OptiSPICE Electrothermal Simulation Verification}

The thermal models produced by Atar for this thesis were coupled into the OptiSPICE electrical models to provide linked electrothermal simulation capability. To determine if these electrothermal simulations produced results that matched the electrical and thermal behaviour of the physical devices, GaN Systems provided example devices with 2 known cells intentionally deactivated for testing and comparing to simulations. Since the simulations were only set up for steady-state conditions, to compare the simulations to a physical device it was necessary to power the example chips in DC. The two biggest concerns with operating the devices in DC were providing the high power needed and managing the high temperatures generated as a result.

\section{Physical Device Testing Setup}

For providing power to the mounted devices, the KEPCO RA55 rack with KEPCO MST power supplies shown in Figure 3.12 were used. This rack had eight individual, 


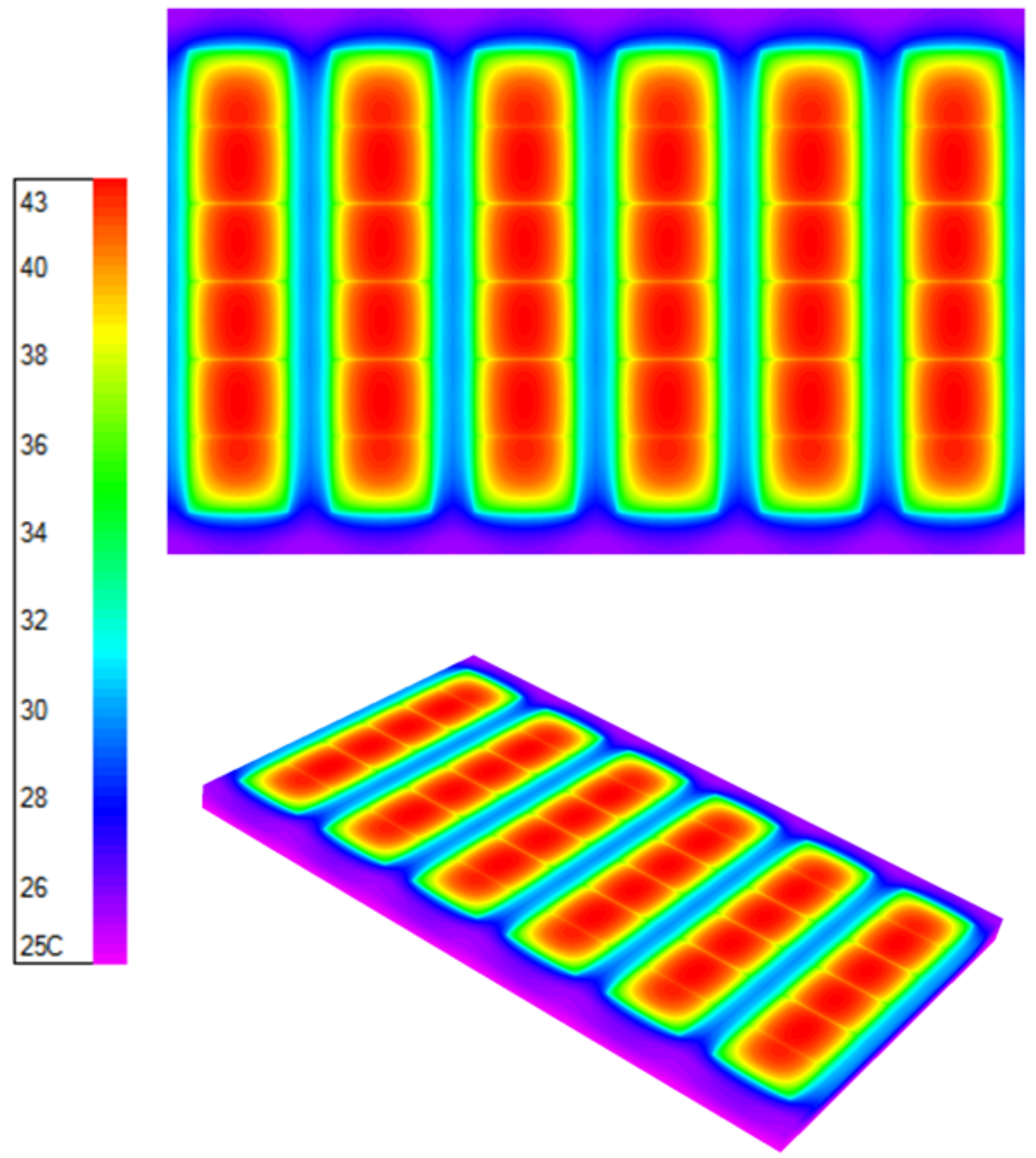

Figure 3.10: Atar thermal simulation results for comparison to the GaN Systems results shown in Figure 3.11. 


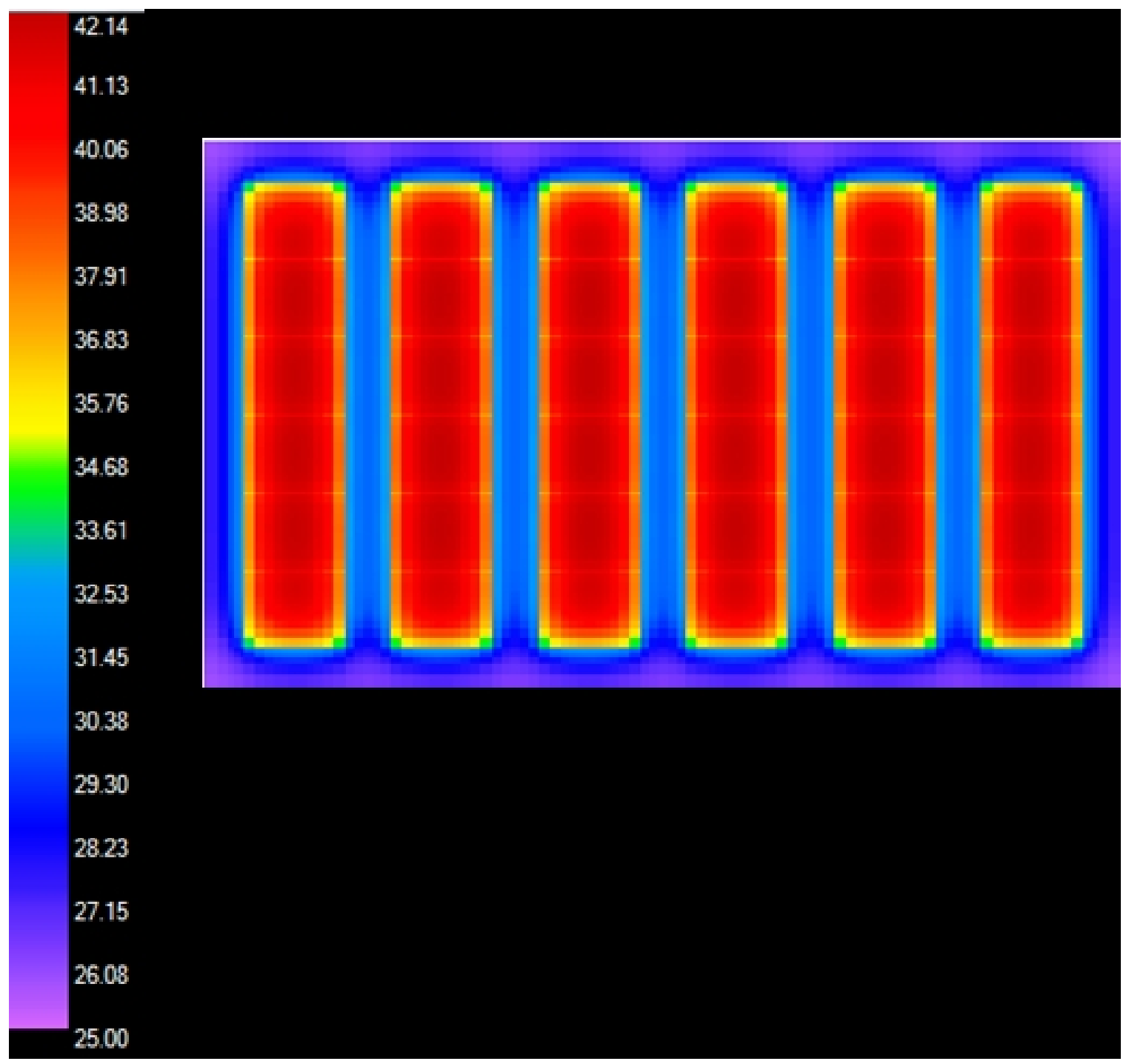

Figure 3.11: GaN Systems commercial thermal simulation results for comparison to Atar results as shown in Figure 3.10. 
computer-controllable power supplies (two $25 \mathrm{~V} / 8 \mathrm{~A}$, five $15 \mathrm{~V} / 12 \mathrm{~A}$, and one $6 \mathrm{~V} / 24 \mathrm{~A}$ ) that could be connected in series or parallel to provide higher voltage or current. Originally it was planned to run the devices at high voltage, low current with the supplies connected in series but this caused issues with output faults in the supplies which required constant power-cycling to reset the error. It was then decided to operate the devices in low voltage, high current and the two $25 \mathrm{~V} / 8 \mathrm{~A}$ supplies were connected in parallel to provide a maximum of $25 \mathrm{~V}$ and $16 \mathrm{~A}$ which provided a more stable operation for the supplies.

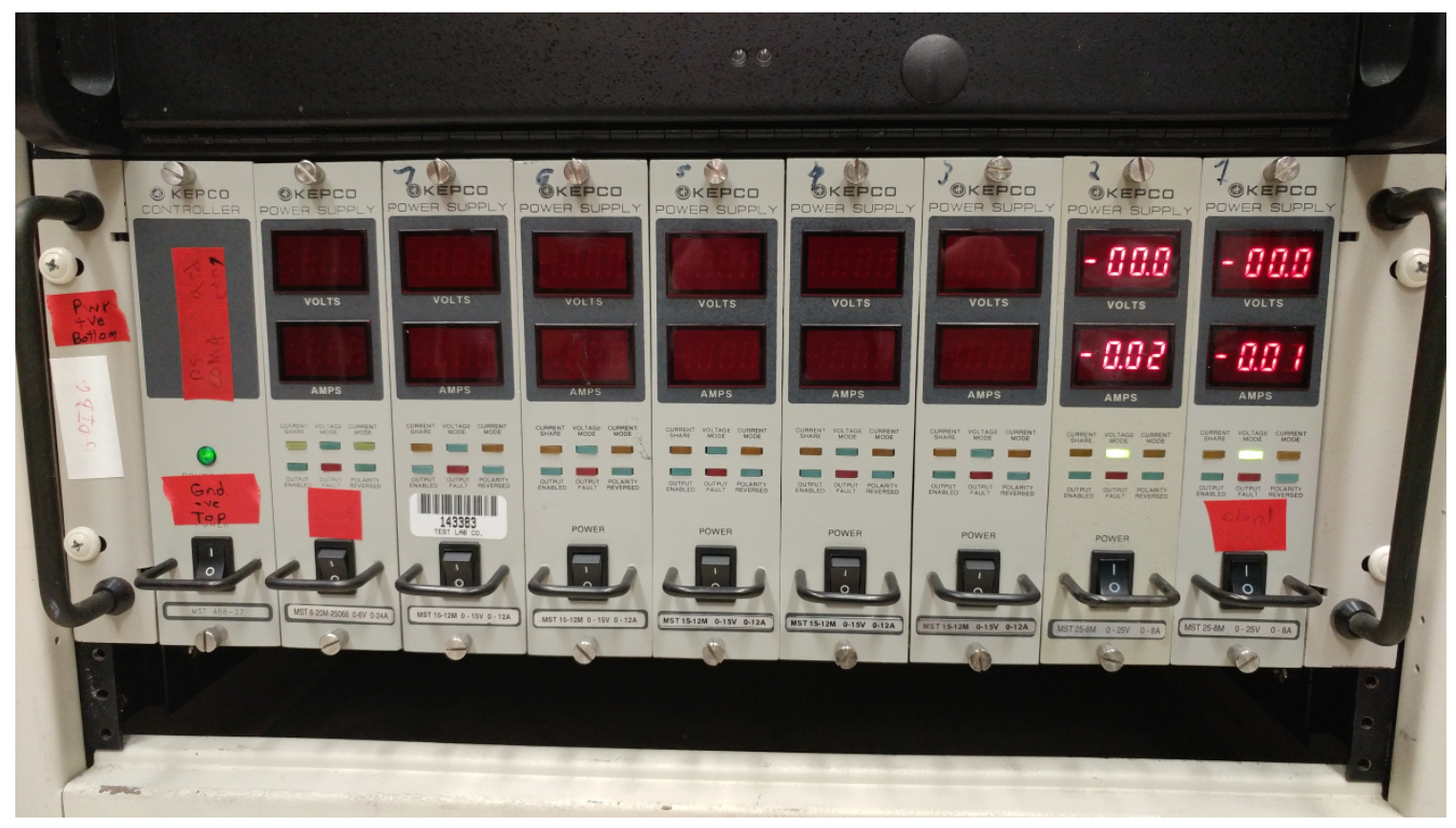

Figure 3.12: KEPCO RA55 rack mount with MST power supplies used for the high voltage/high current powering of the GaN devices.

The outputs on the rear of the KEPCO rack had connectors that were nonstandard and difficult to acquire. Before this project, the connections were simply made with bare 14AWG solid core wire pushed in the connector contacts as shown in Figure 3.13. In the interest of operating the supplies in a safer manner, especially considering the original intent to use high voltage for the tests, connectors were designed and built so that connections could be made with banana plugs. The 3D model of the connector design is shown in Figure 3.14 and the final assembled connector is 
shown in Figure 3.15. Eight connectors were made despite only needing to use two supplies for the test, shown connected to the supplies in Figure 3.16.

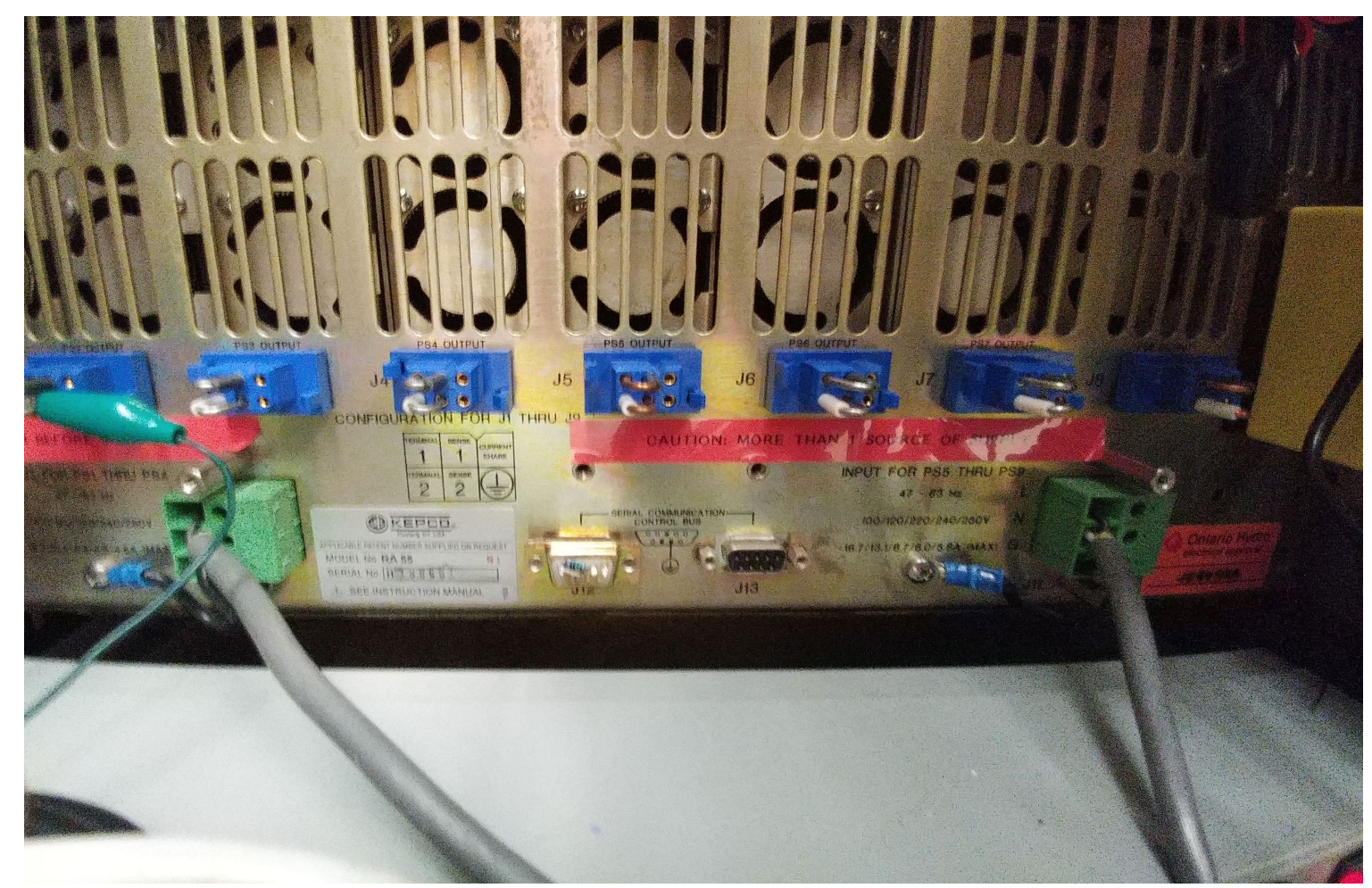

Figure 3.13: KEPCO RA55 original power supply connectors, where connections were made with bare 14AWG solid core wires.

With the power supply issues resolved, a way to keep the devices cool during operation was the next step. For actively cooling the devices, a Temptronic TPO315B temperature controller was used. The controller is shown in Figure 3.17 and is connected to the thermal chuck mounted in the Karl Suss wafer probe station shown in Figure 3.18. To attach the devices to the chuck, 1/4" aluminium heat sink blocks measuring $2 " \times 2$ " were designed with a printed circuit board (PCB) epoxied to the surface with split copper pads for connecting the source and drain of the devices to the power supply through a pin header. A cavity routed in the PCB allowed for the devices to be attached directly to the aluminium block using Epotek H20E silver epoxy as the thermal interface material (TIM). The heat sink with PCB and mounted but unconnected device is shown in Figure 3.19. The heat sink also had a hole drilled 

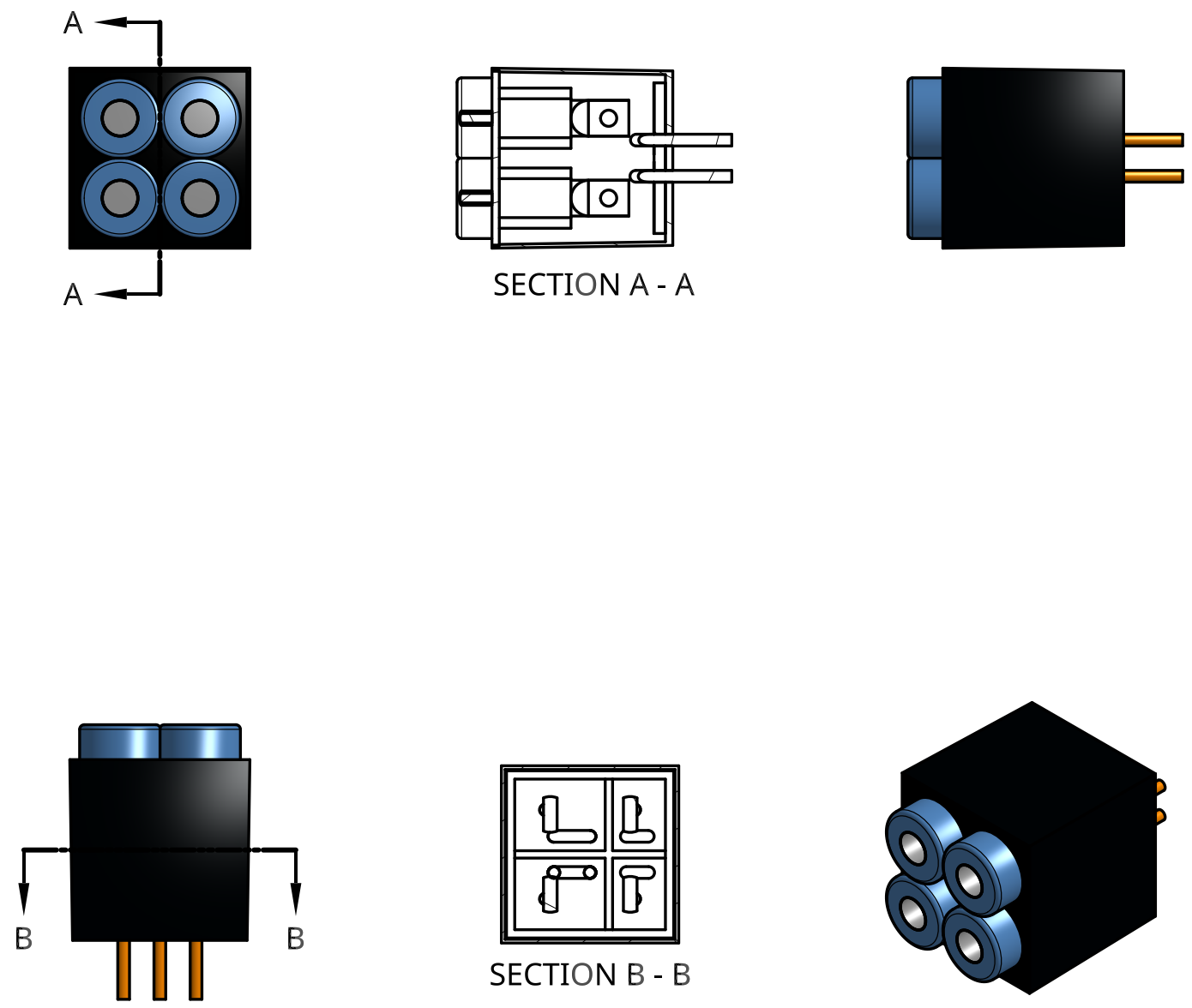

Figure 3.14: 3D CAD model of the connector designed for high voltage testing with banana plug inputs for the KEPCO RA55. 


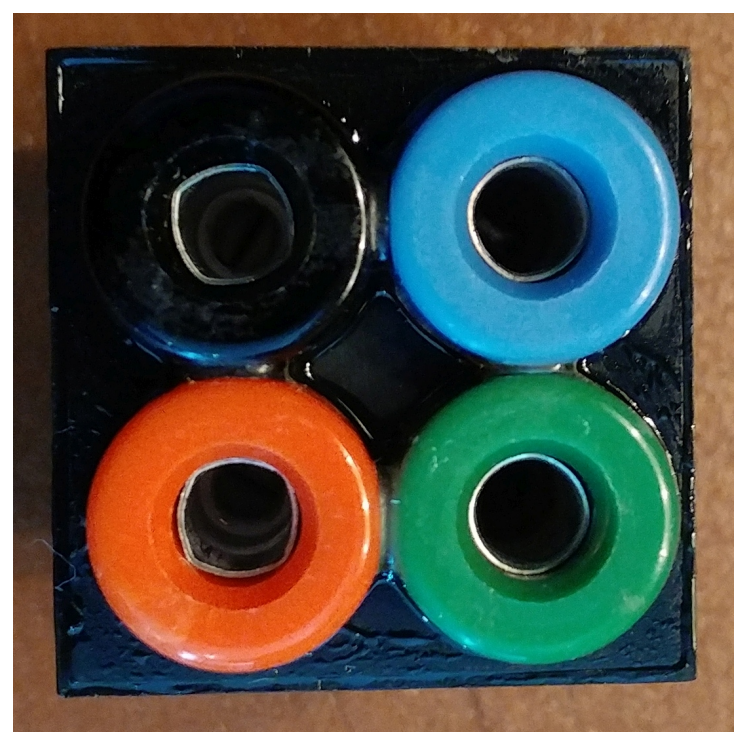

(a) Connector Top View

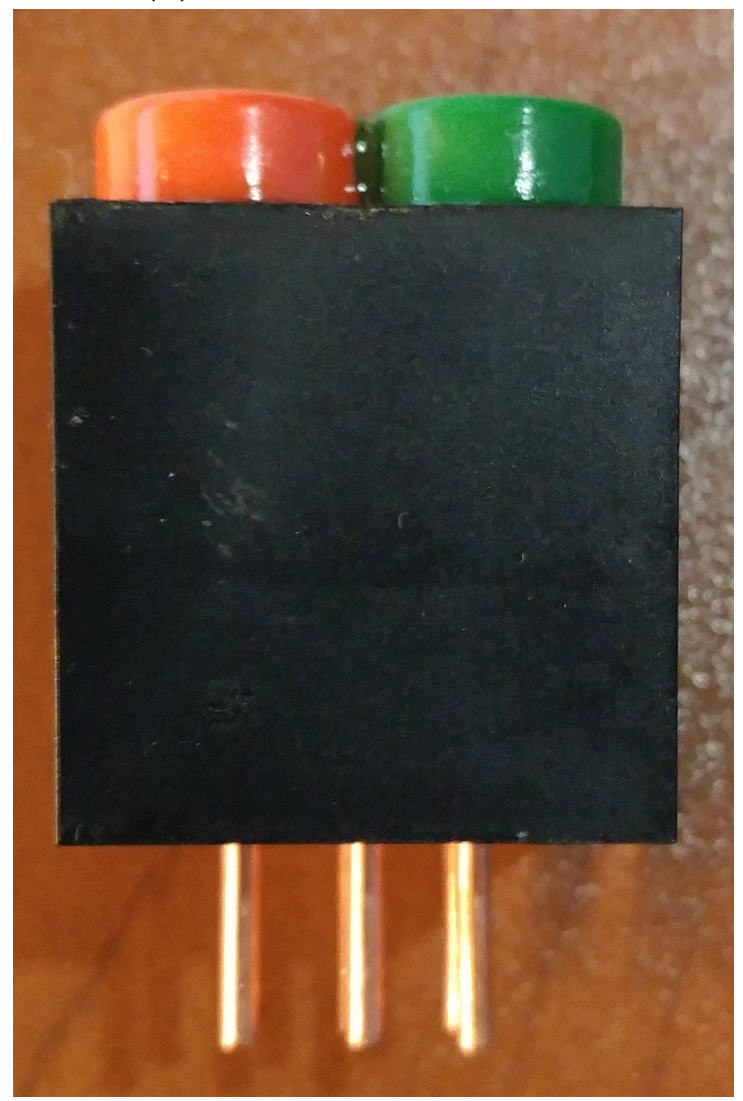

(c) Connector Front View

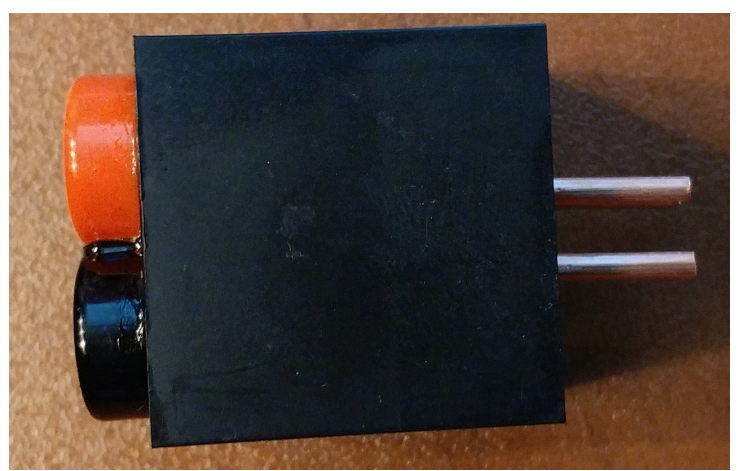

(b) Connector Side View

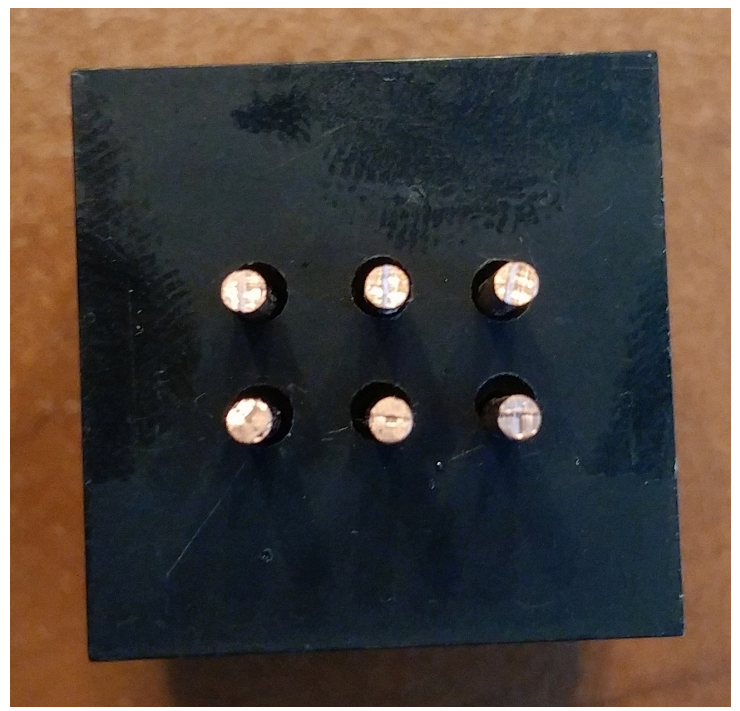

(d) Connector Bottom View

Figure 3.15: The completed KEPCO RA55 power supply connector based on the design in Figure 3.14 


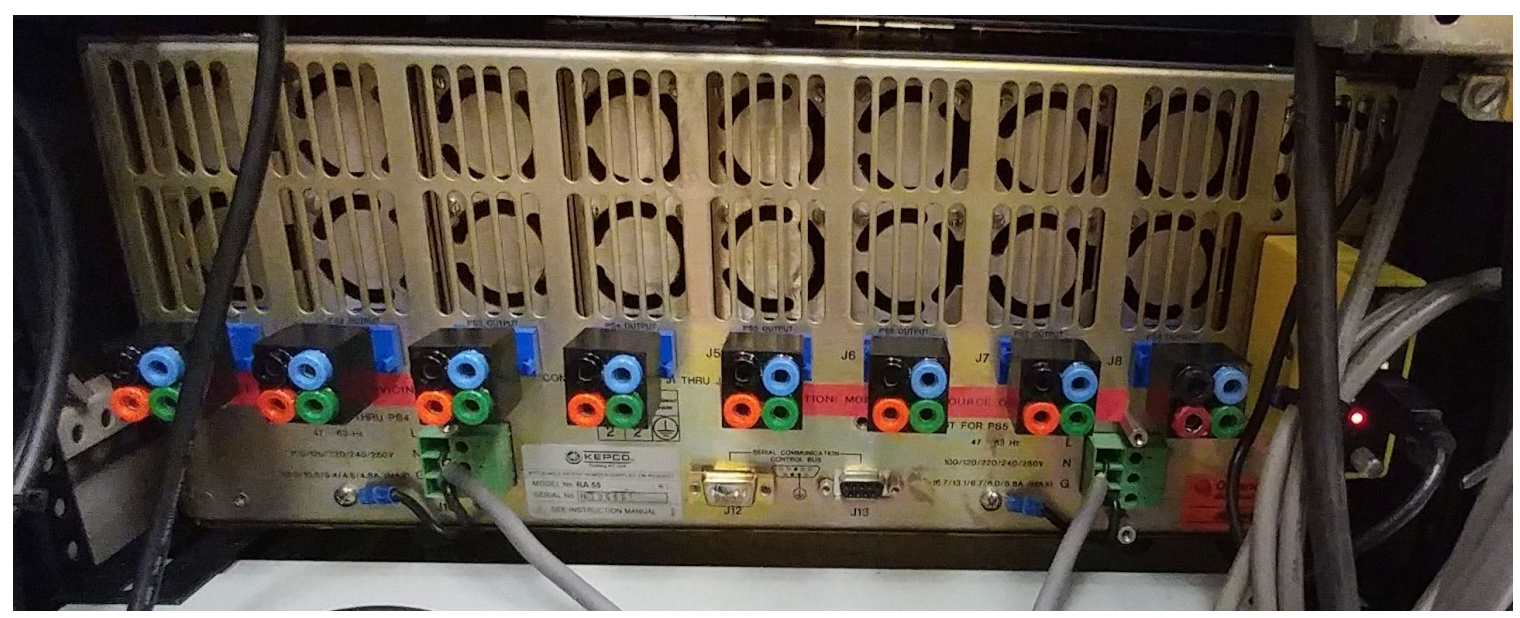

Figure 3.16: KEPCO RA55 power supply unit with the new connectors for high voltage testing with banana plug inputs.

into the aluminium near the mounted device to monitor the temperature of the block surface with a thermocouple.

With the ways to power and cool the devices established, next was preparing the devices for testing. Two device conditions were considered: fully packaged devices would be used to establish operating conditions since they were simple to mount to the heat sink blocks for quick replacement and a polished device to be able to view the thermal distribution of the actual individual island transistors. The fully packaged device was an untouched device mounted on the aluminium heat sink with the source and drain pads connected to the split pads of the PCB. Several other devices were processed in order to try and observe the thermal behaviour of the individual cells. Since the individual cells would not be visible using thermal imaging due to the heat spread through the upper device layers, fine grit polishing paper was used on the Buehler ECOMET III Polisher/Grinder shown in Figure 3.20 to mechanically polish the upper layers down to the copper RDL on the surface of the chip embedded in the package. These upper layers consisted of the package metal and copper pillar interconnects as detailed in the patent for GaN Systems device packaging [1]. This left the bare chip die exposed with the plan of connecting to the copper RDL. The full polished device is shown in Figure 3.21a with a close up of just the chip shown 


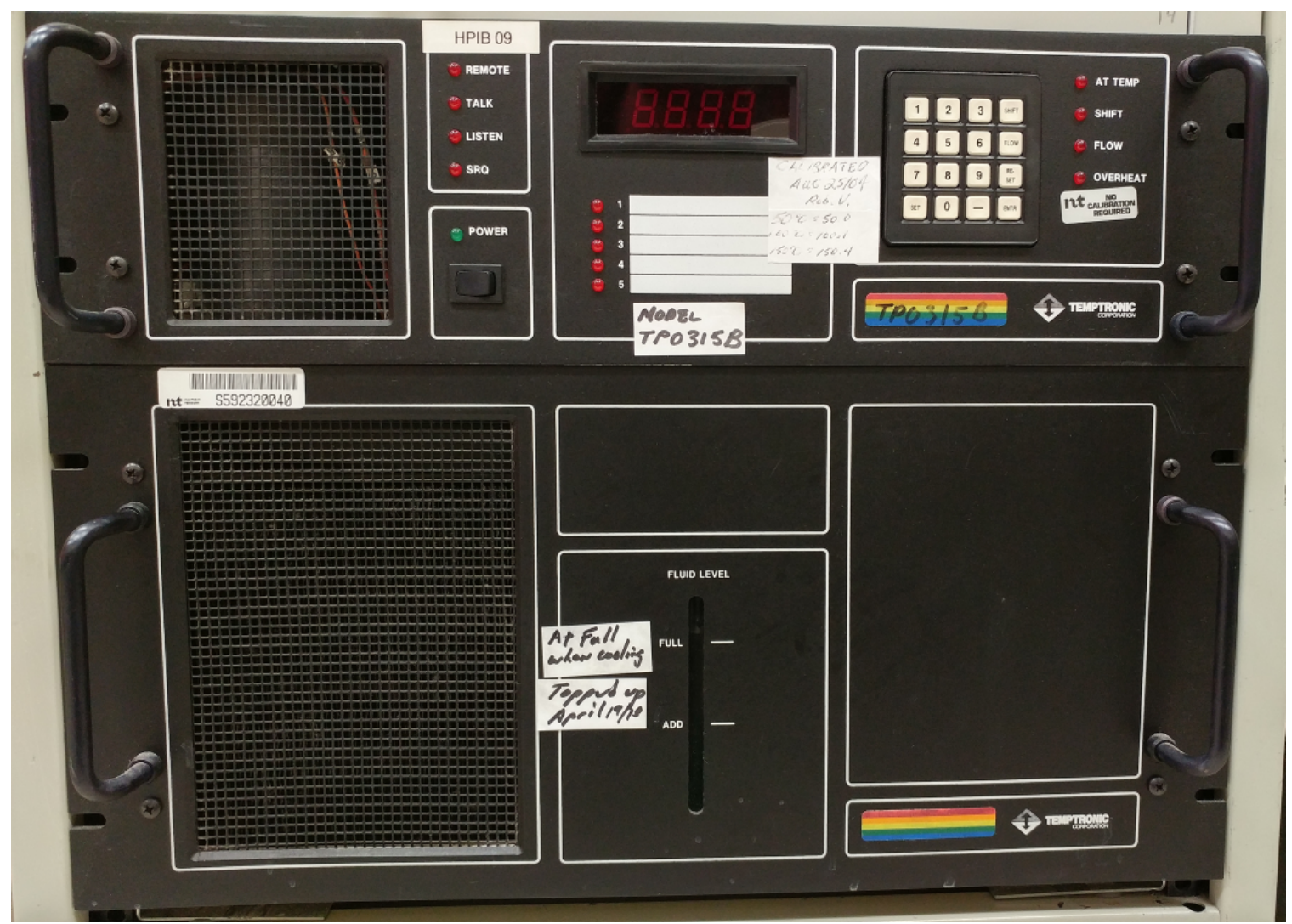

Figure 3.17: Temptronic TPO315B temperature controller used for cooling the chuck during testing.

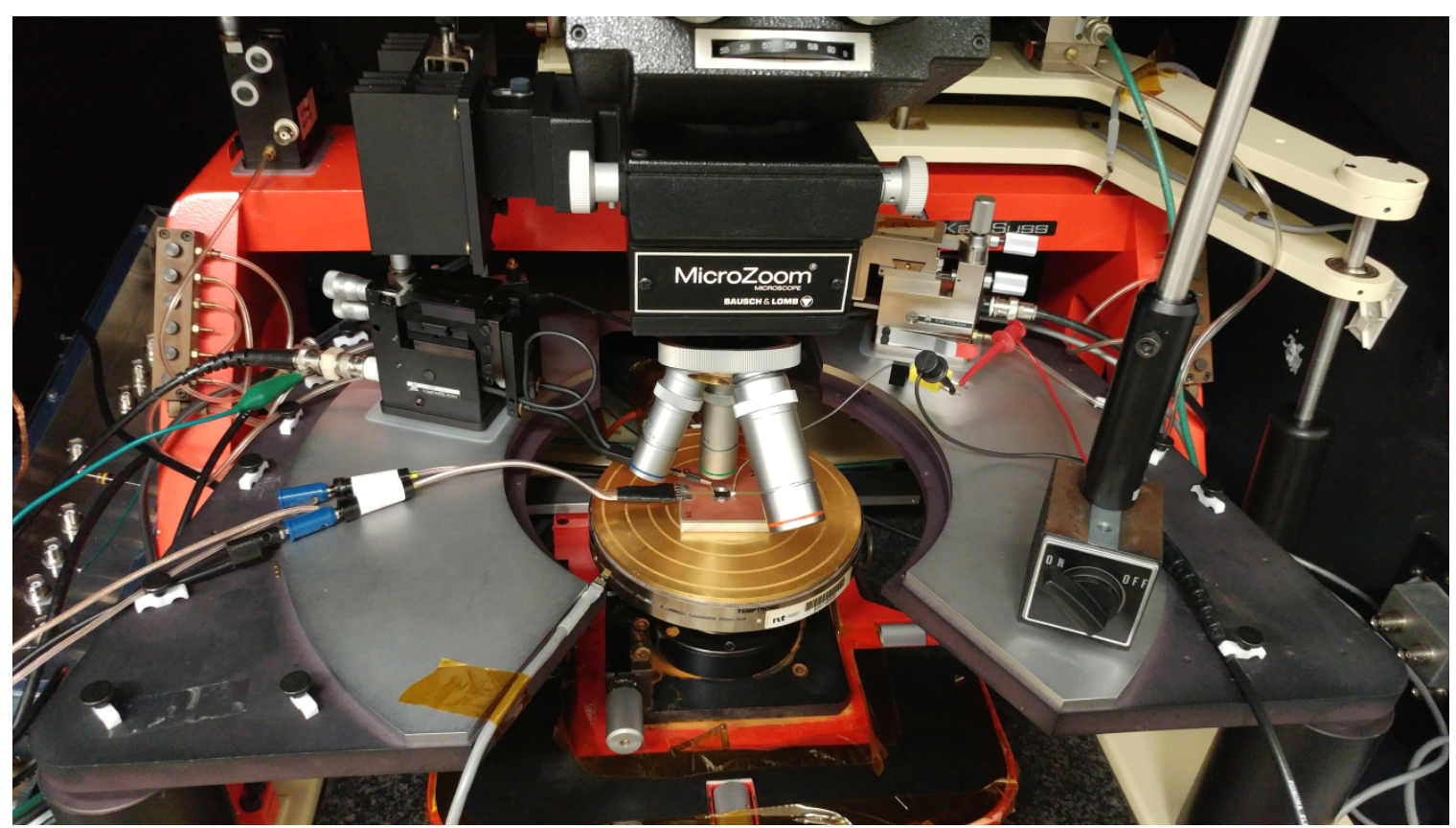

Figure 3.18: Karl Suss probe station with temperature controlled chuck. 

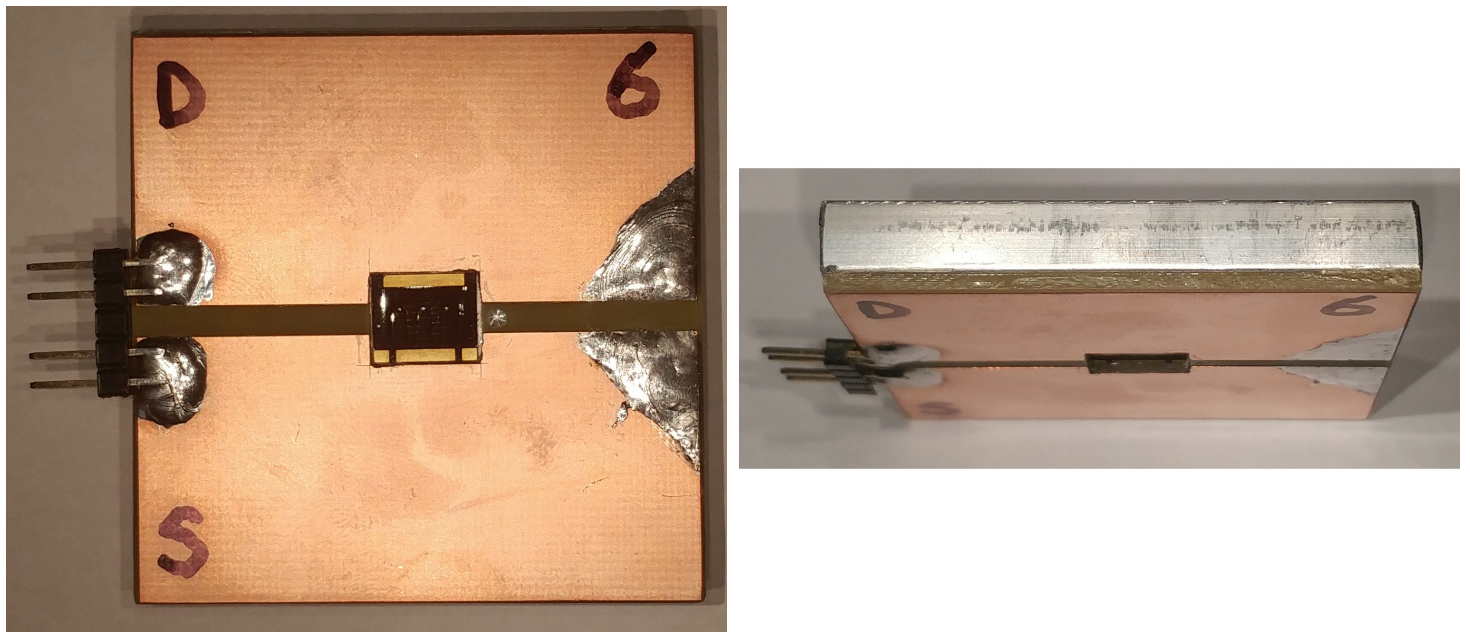

(a) Top view of aluminium heat sink block with PCB mounted and chip in cavity.

(b) Side view of aluminium heat sink block with PCB.

Figure 3.19: $2 " \times 2 " \times 1 / 4$ " aluminium heat sink block used to dissipate heat away from the device during testing.

in Figure 3.21b.

\section{Initial Physical Test Failures}

The first tests of the unpolished devices to determine the operating conditions that were feasible ended up destroying several devices, with one test melting the epoxy of the package of the device as the junction temperature climbed to levels that caused catastrophic damage. This particular failed chip is shown in Figure 3.22, with a close up image of the major failure point shown in an optical and a scanning electron microscope image.

\section{Establishment of Backside Temperature for Simulations}

From the first failed tests, it was concluded that keeping the backside at the $25^{\circ} \mathrm{C}$ specified by the earlier simulations would not be feasible even with the liquid cooling provided by the temperature controlled chuck. In order to compare the temperature 


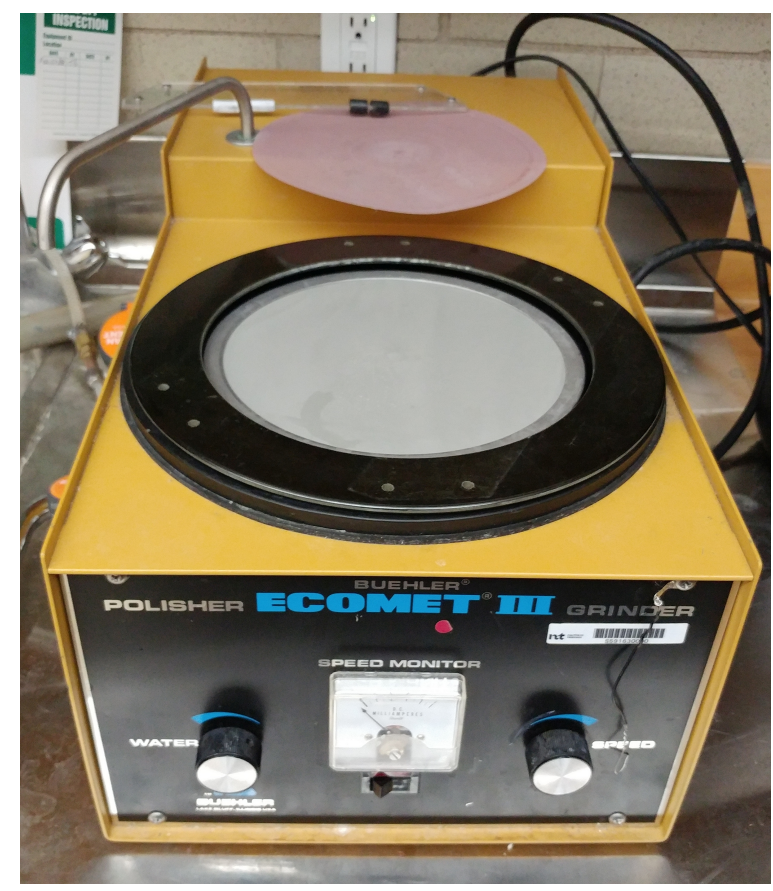

Figure 3.20: Buehler ECOMET III polisher/grinder used for mechanically polishing the upper layers of the device down to the copper RDL on the chip surface.

distributions from the electrothermal simulations to a physical test device, a more realistic backside temperature was necessary. By operating the device at a low enough power that the chuck was able to hold a steady temperature, data was gathered to characterize the thermal system. From this data, it was possible to approximately determine a backside temperature to set in the simulations to give a reasonable comparison to the physical testing.

For the successful low power test, the drain voltage of the device was set to $12 \mathrm{~V}$ (the actual drain voltage was measured to be $11.25 \mathrm{~V}$ due to resistive losses in the connections) and the gate voltage was set to $2 \mathrm{~V}$ while the temperature controlled chuck was set to cool the chuck to $5^{\circ} \mathrm{C}$. The device was powered and held at the same gate and drain voltage to allow the device to settle at a thermal equilibrium. The plot of the heat sink surface temperature and the power dissipated in the device over time is shown in Figure 3.23. The FLIR T1020 infrared camera, which was calibrated and provided by FLIR Systems, was used for taking thermal images of the devices 


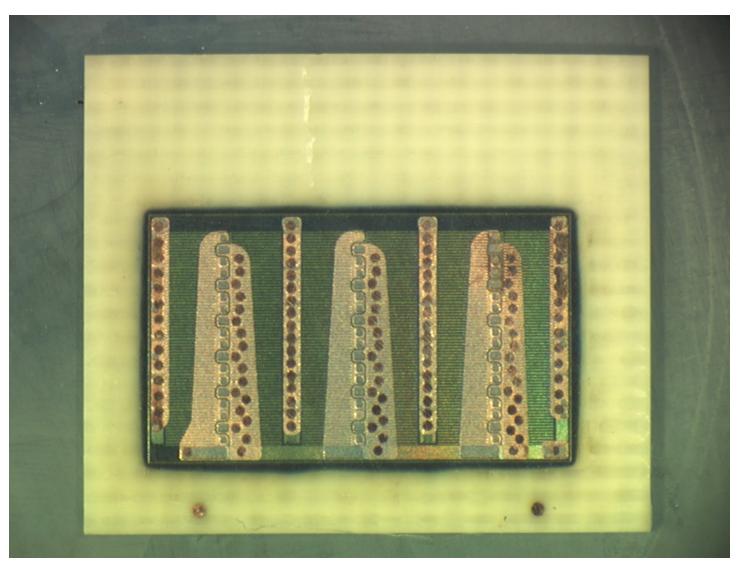

(a) Full view of polished device surrounded by packaging material.

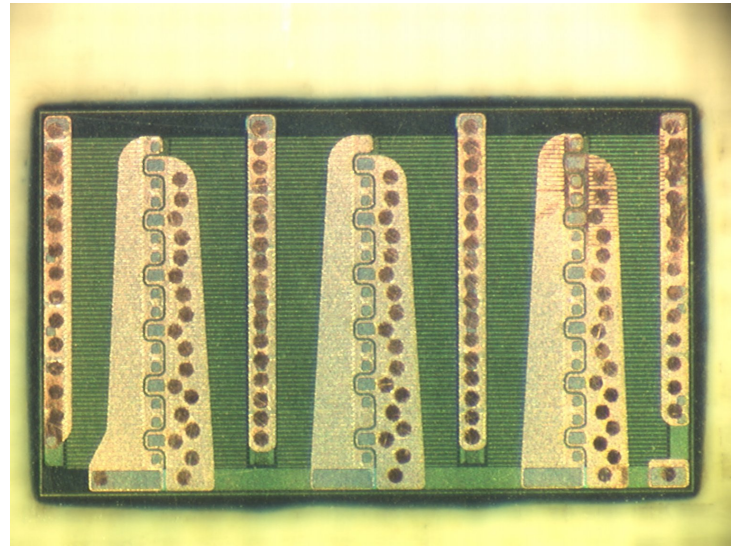

(b) Close-up view of polished chip die showing copper RDL and individual cells.

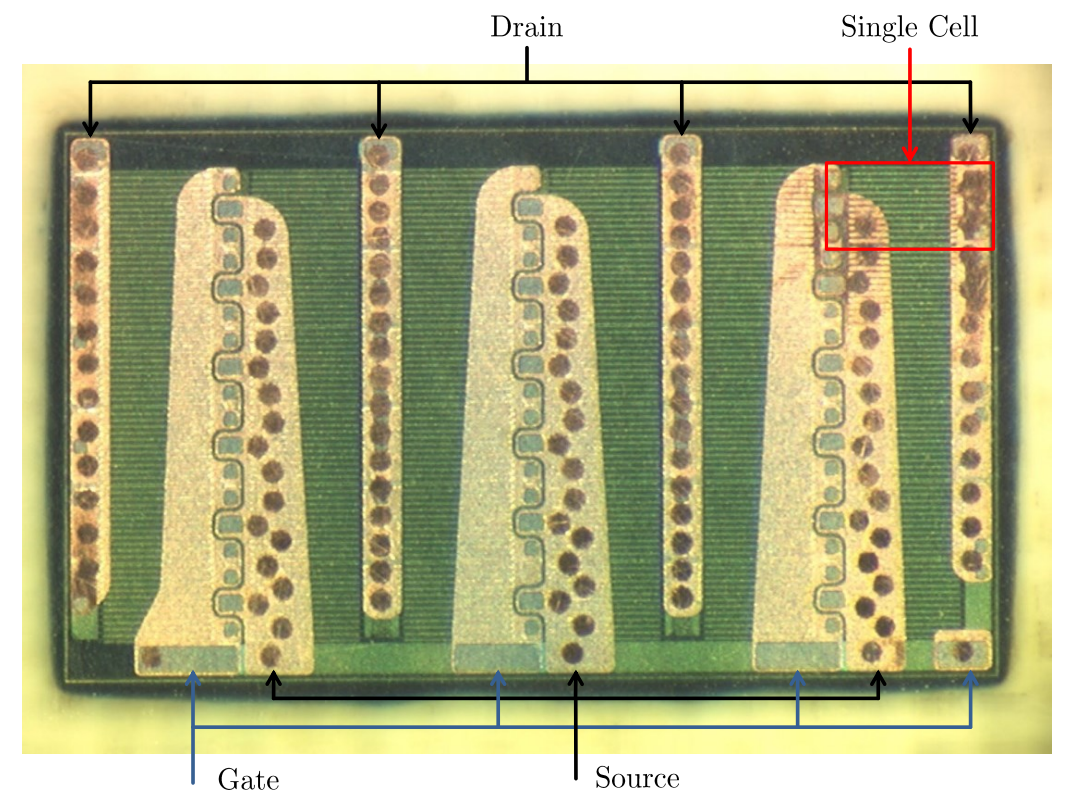

(c) Terminal connections of RDL and indication of cell area visible.

Figure 3.21: Images of a device after mechanical polishing showing the copper RDL and identifying connections. 


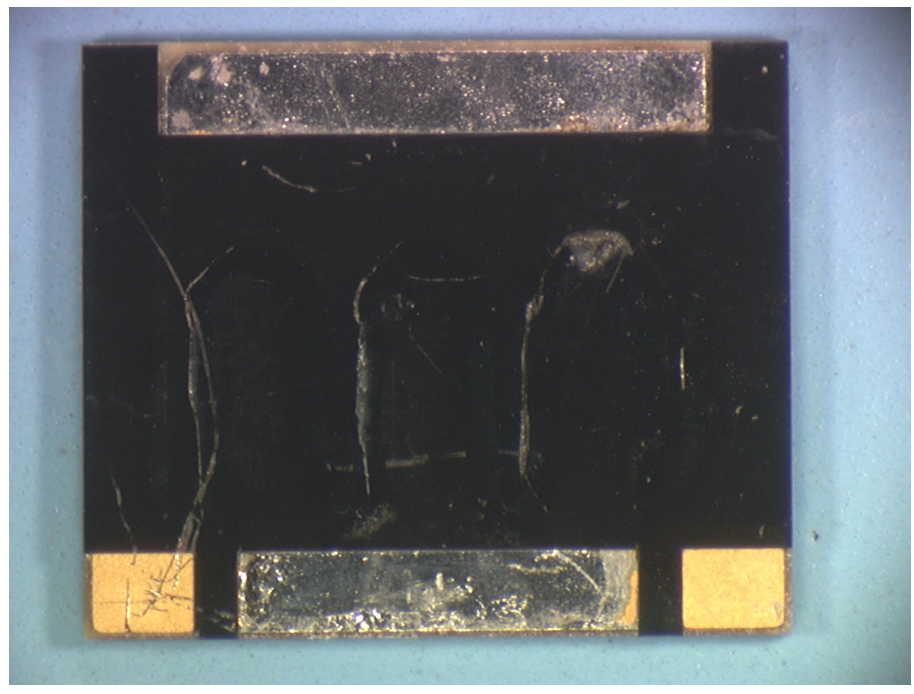

(a) Failed test of unpolished device - Overall device.

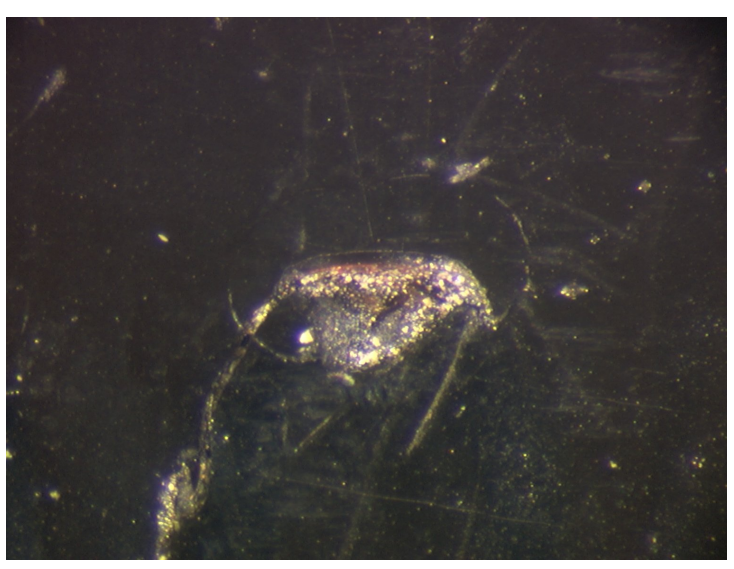

(b) Failed test of unpolished device Optical zoom.

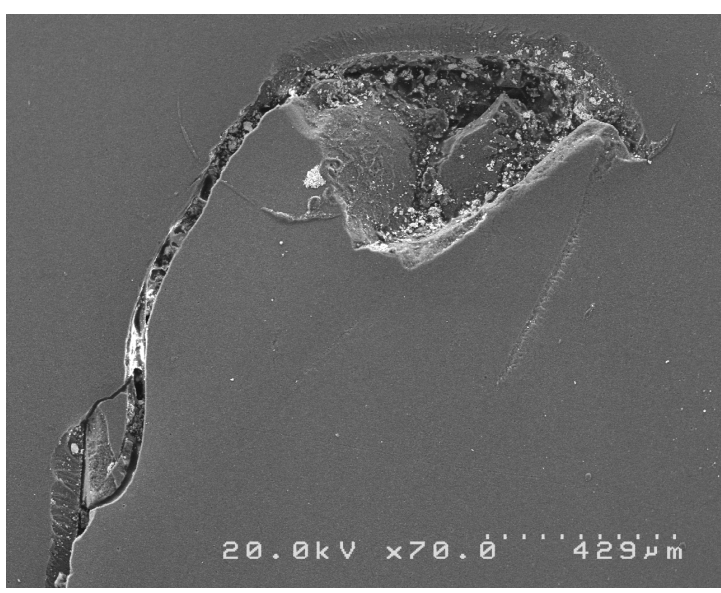

(c) Failed test of unpolished device Scanning electron microscope image.

Figure 3.22: A failed unpolished device after testing which resulted in a melted package due to overheating. 
in operation. The emissivity was set to 0.85 on the camera, which was necessary to obtain accurate results. The emissivity of an object being photographed may cause improper temperature readout if the object in question has a low emissivity and the camera is not calibrated properly. This would cause the temperature to appear lower than it actually is as less thermal radiation is received by the camera. The thermal image of the surface of the unpolished device at equilibrium is shown in Figure 3.24. The highest temperature measured on the surface, $120.6{ }^{\circ} \mathrm{C}$, is marked by the crosshair. This value for the top surface was assumed to be correct as the camera was calibrated before use by FLIR Systems and the emissivity of the surface of the device (a gloss black coating) should be around 0.87. At the time the picture was taken the top of the aluminium block temperature was measured to be 28.21 ${ }^{\circ} \mathrm{C}$ and the Temptronic temperature controller reported the chuck temperature to be $18.1^{\circ} \mathrm{C}$.

The information provided by the successful test of the unpolished device allowed for the analysis of the thermal circuit for the setup in order to determine the approximate temperature of the backside of the device. A graphical explanation of the temperatures and thermal resistances of the test setup is shown in Figure 3.25.

Since the top of the chip was not connected to any sort of heat sink (relying only on radiation and convection for any cooling), this node was treated as 'floating' and removed from the circuit meaning the observed surface temperature was considered as the junction temperature for the analysis. The other known or measurable quantities are the power dissipated, chuck temperature, block temperature, and the junction to case thermal resistance of the GS66516T device as specified by the datasheet. Using these values it is possible to analyze the thermal circuit to determine the backside temperature $\left(\mathrm{T}_{\text {Case }}\right)$ to set in the simulations for comparison. The solved thermal circuit is shown in Figure 3.26. A table of these values and a description of how they were obtained is presented in Table 3.4. 


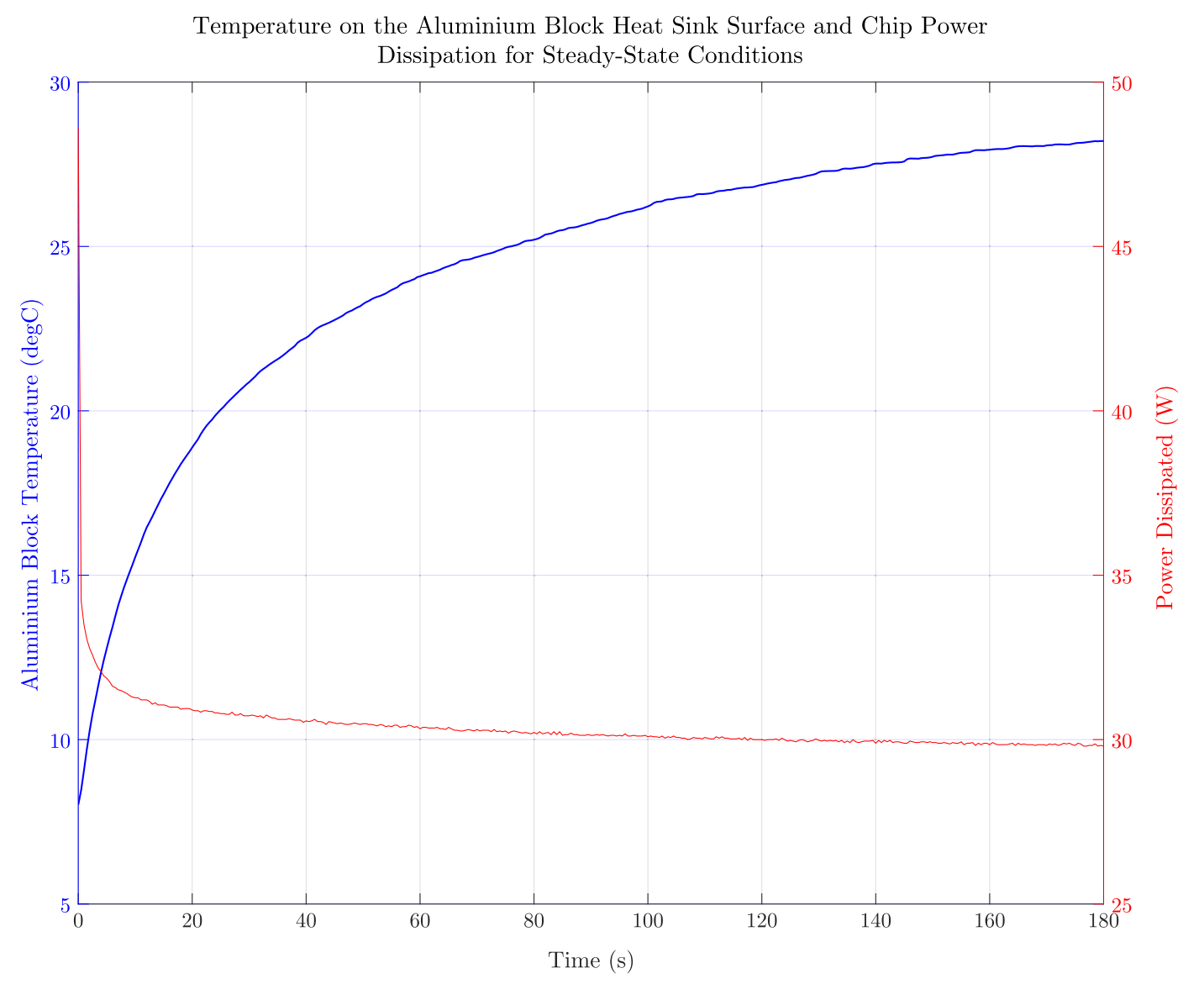

Figure 3.23: Aluminium block temperature and chip power dissipation at steady-state over time. 


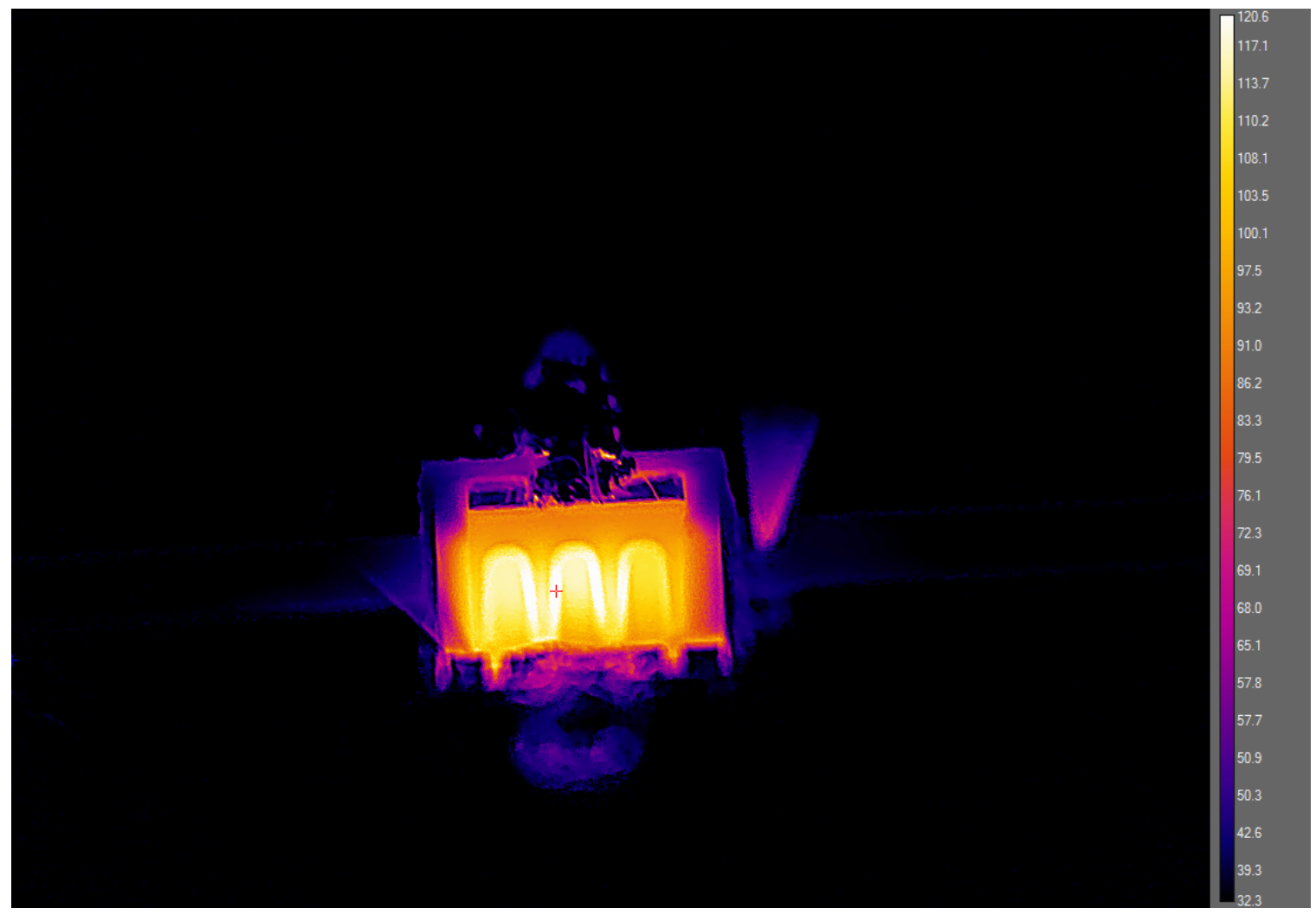

Figure 3.24: Thermal image of an unpolished device dissipating $30 \mathrm{~W}$ of power (legend in units of ${ }^{\circ} \mathrm{C}$ ).

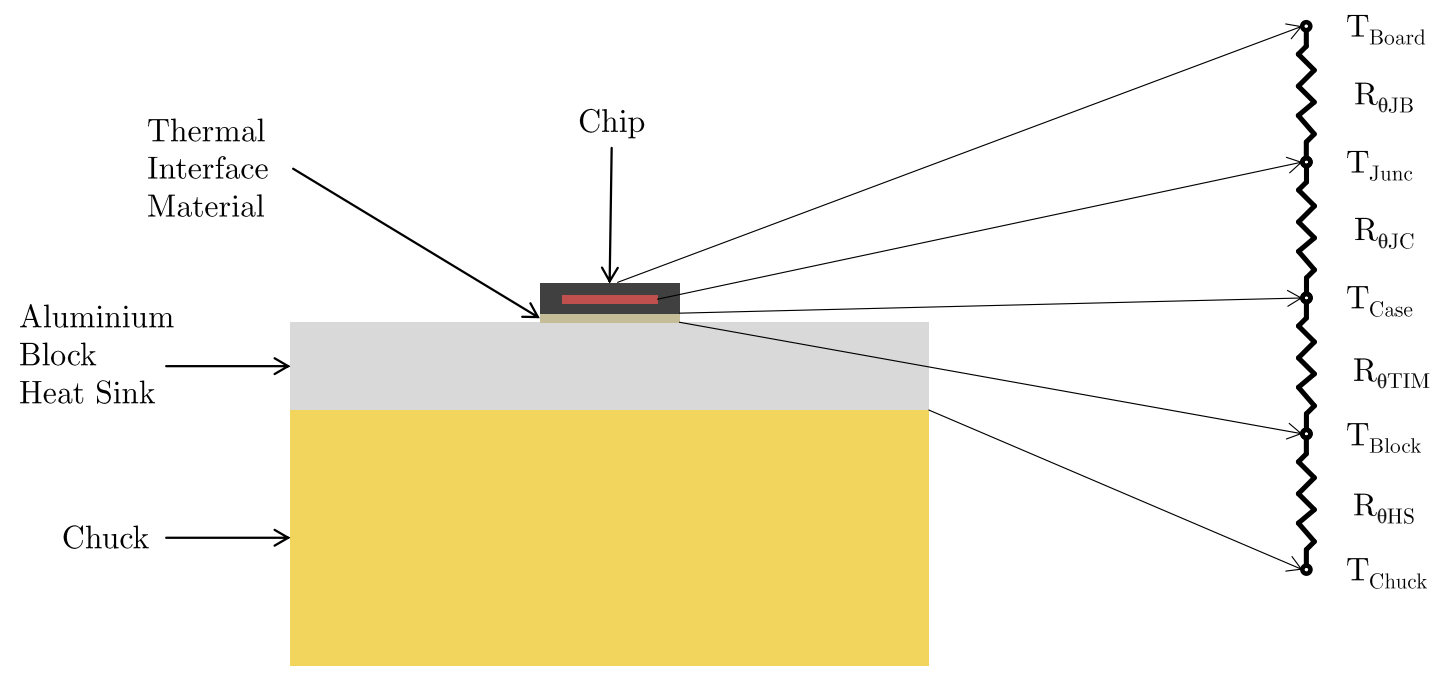

Figure 3.25: A graphical representation of the setup used for the thermal analysis. 


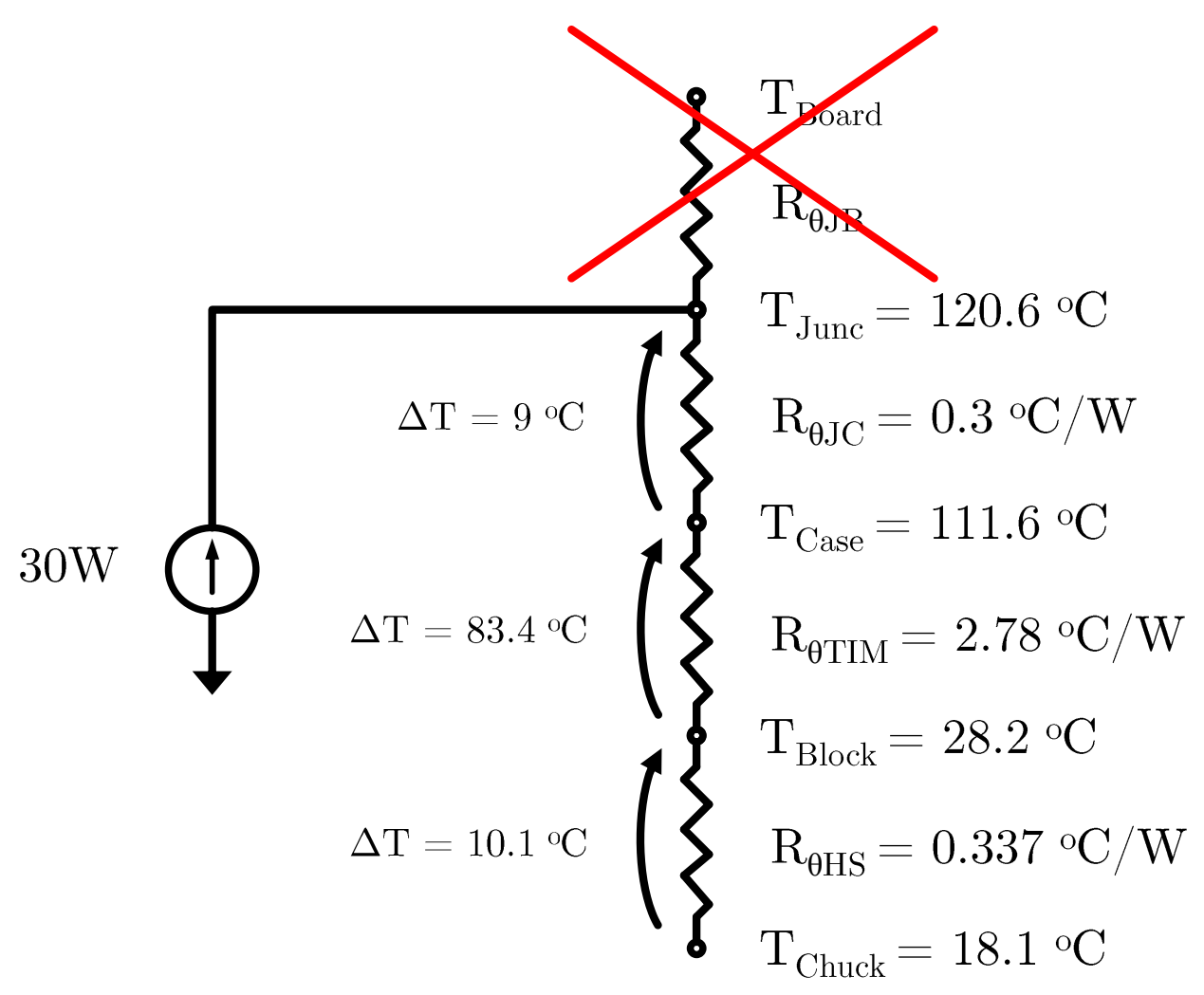

Figure 3.26: The thermal analysis representing the physical testing of the device. 
Table 3.4: Results of thermal analysis based on physical test data.

\begin{tabular}{|l||c|l|}
\hline Parameter & Value & Value From? \\
\hline $\mathrm{T}_{\text {Junc }}\left({ }^{\circ} \mathrm{C}\right)$ & 120.6 & Measurement: FLIR T1020 \\
\hline $\mathrm{R}_{\theta \mathrm{JC}}\left({ }^{\circ} \mathrm{C} / \mathrm{W}\right)$ & 0.3 & Specification: Datasheet $(\mathrm{GS} 66516 \mathrm{~T})$ \\
\hline $\mathrm{T}_{\text {Case }}\left({ }^{\circ} \mathrm{C}\right)$ & 111.6 & Calculation: $\mathrm{T}_{\text {Junc }}-\Delta \mathrm{T}_{\mathrm{JC}}$ \\
\hline $\mathrm{R}_{\theta \mathrm{TIM}}\left({ }^{\circ} \mathrm{C} / \mathrm{W}\right)$ & 2.78 & Calculation: $\Delta \mathrm{T}_{\mathrm{TIM}} \div \mathrm{P}_{\mathrm{D}}$ \\
\hline $\mathrm{T}_{\text {Block }}\left({ }^{\circ} \mathrm{C}\right)$ & 28.2 & Measurement: Thermocouple \\
\hline $\mathrm{R}_{\theta \mathrm{HS}}\left({ }^{\circ} \mathrm{C} / \mathrm{W}\right)$ & 0.337 & Calculation: $\Delta \mathrm{T}_{\mathrm{HS}} \div \mathrm{P}_{\mathrm{D}}$ \\
\hline $\mathrm{T}_{\text {Chuck }}\left({ }^{\circ} \mathrm{C}\right)$ & 18.1 & Measurement: Temptronic TPO315B \\
\hline $\mathrm{P}_{\mathrm{D}}(\mathrm{W})$ & 29.813 & Calculation: $\mathrm{V}_{\mathrm{D}} * \mathrm{I}_{\mathrm{D}}$ \\
\hline$\Delta \mathrm{T}_{\mathrm{JC}}\left({ }^{\circ} \mathrm{C}\right)$ & 9 & Calculation: $\mathrm{P}_{\mathrm{D}} * \mathrm{R}_{\theta \mathrm{JC}}$ \\
\hline$\Delta \mathrm{T}_{\mathrm{TIM}}\left({ }^{\circ} \mathrm{C}\right)$ & 83.4 & Calculation: $\mathrm{T}_{\text {Case }}-\mathrm{T}_{\text {Block }}$ \\
\hline$\Delta \mathrm{T}_{\mathrm{HS}}\left({ }^{\circ} \mathrm{C}\right)$ & 10.1 & Calculation: $\mathrm{T}_{\text {Block }}-\mathrm{T}_{\text {Chuck }}$ \\
\hline
\end{tabular}

\section{Thermal Imaging of Polished Device}

To compare the thermal distribution that Atar produced to the example device, connections to the RDL had to be made in order to provide power. After various unsuccessful attempts to attach wires to the RDL with both a wire bonding machine and using H20E silver epoxy to bond fine gauge wire to the traces, the H20E silver epoxy was used to create a bus between the traces to which a heavier gauge wire was attached as shown in Figure 3.27a. A close-up of the silver epoxy connection traces is shown in $3.27 \mathrm{~b}$.

Unfortunately, after thermal imaging the polished devices, two issues were discovered. The first issue occurred with the device used for the test. At some point between the preliminary testing to establish that the device was functioning to the final test of the polished device, approximately one third of the device appears to have failed. This can be seen in the infrared image shown in Figure 3.28 as the right side of the device decreases drastically in temperature. This corresponded to a decrease in 


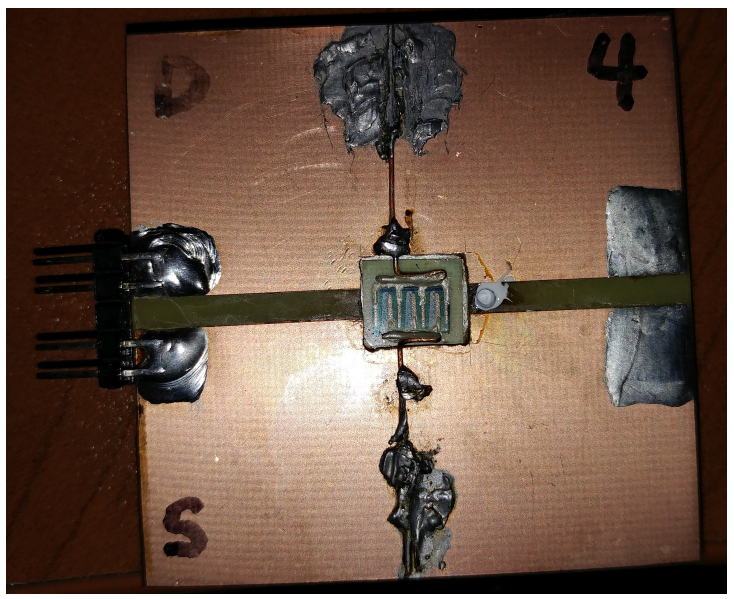

(a) Polished device on heat sink

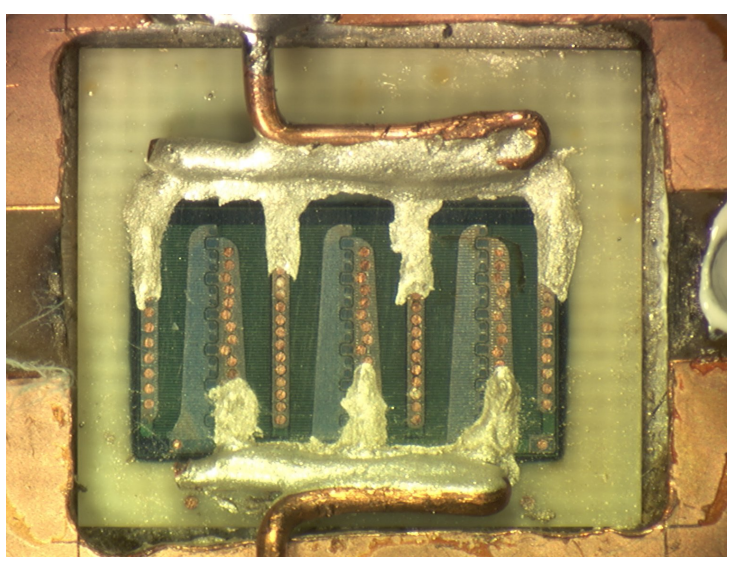

(b) Close-up of chip connections.

Figure 3.27: A polished device mounted to the heat sink block showing connections made for testing.

current output for the same operating conditions used to test the unpolished device. The possible cause for this failure could be due to damage from the mechanical polishing, electrostatic discharge damage due to handling, or possibly thermal damage from the heating of the device to cure the silver epoxy.

The second issue encountered was the lack of discernibility of the individual cells in the polished device in the infrared spectrum. As can be seen in Figure 3.28, individual cells cannot be seen as the thermal image appears. As detailed in the packaging patent [1] and the fault tolerance patent [6] from GaN Systems, the layer between the on-chip metallization and the copper RDL may be polyimide. Due to either thermal spreading or optical scattering in the infrared spectrum, the cells are optically visible but not visible in the infrared image as the polyimide is almost opaque for the spectral range of the camera $(7.5-14 \mu \mathrm{m})[39]$. Polyimide also goes by the trade name Kapton so to test the infrared transparency of polyimide, a simple experiment involving the imaging of heat due to the dissipation of power in a resistor was carried out. A resistor was powered and imaged with an infrared camera to give Figure 3.29a. This same resistor was then covered in a square piece of 3.5 mil (89 micron) thick Kapton tape and imaged with the same infrared camera giving the 
image seen in Figure 3.29b. A comparison with the resistor half covered is seen in Figure $3.29 \mathrm{c}$ and shows that the details of the thermal image are blurred. This simple test may offer an explanation why the individual cell thermal distribution cannot be seen in the polished device during infrared imaging.

For completeness, an approximation of the thermal distribution for the polished device from the Atar thermal simulation, produced by turning off the right third of the device, is shown alongside the colour-matched thermal image from the physical test in Figure 3.30.

\section{Electrothermal Simulation Correlation}

Though the thermal imaging of the polished device did not prove useful for comparing the exact cell temperatures to those in the electrothermal simulations, it was still possible to compare to the OptiSpice results using the information gathered from the physical test of the unpolished device that was used to establish the backside temperature. From the parameters determined by that testing, simulations were run by setting $V_{D}, V_{G}$, and $T_{C}$ and the results were compared to those gathered from the physical testing of the unpolished device. Using the ResearchIR software from FLIR it was possible to determine the maximum and average junction temperatures through the package connection fingers as shown in Figure 3.31.

The resulting output for the OptiSPICE electrothermal simulation used in this comparison is included for reference in Appendix C.2 and the results of the comparison between the measured and simulated values are collected in Table 3.5. From this result it can be seen that the error between the simulated and measured values is low enough to consider the electrothermal simulations accurate. 


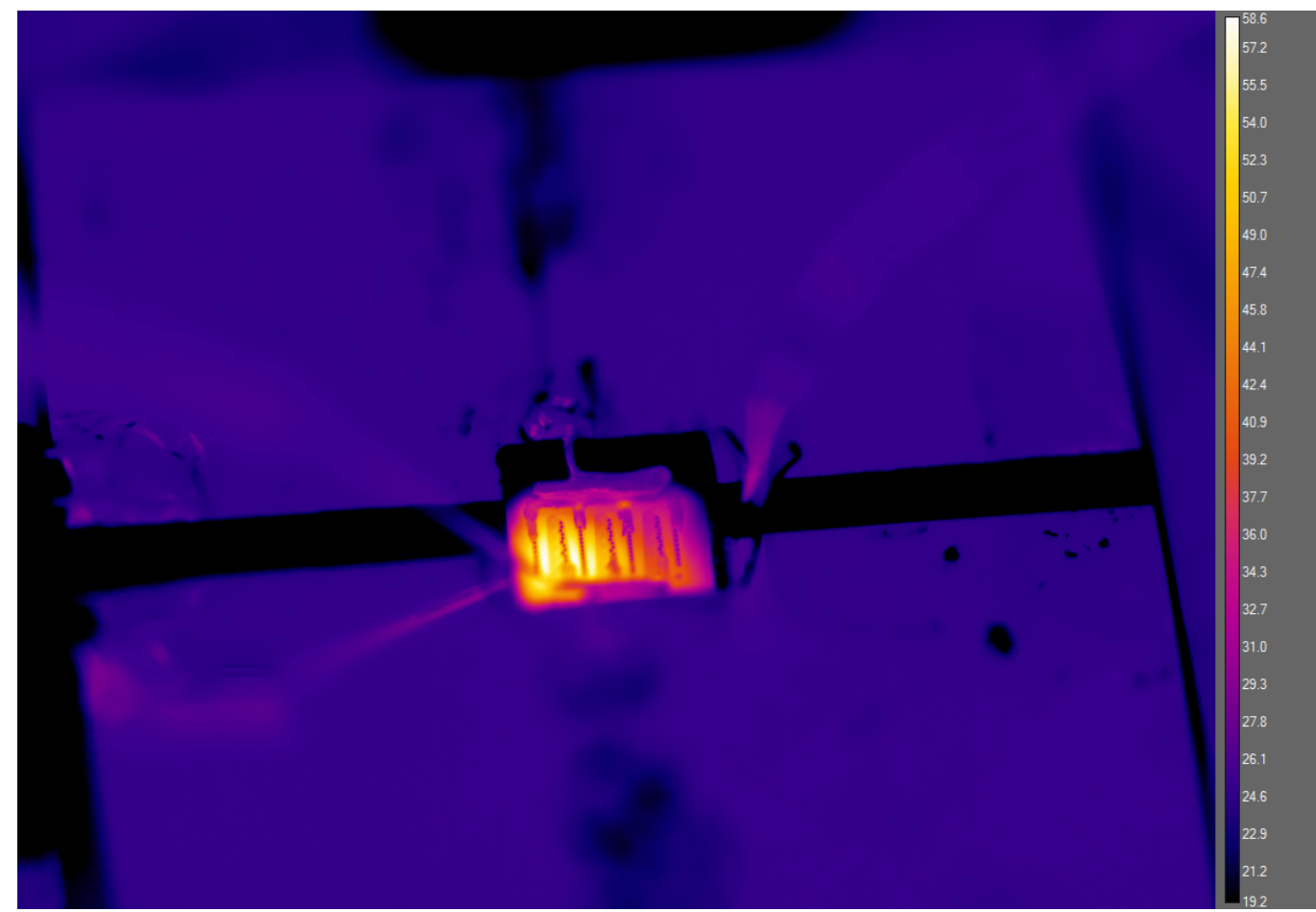

(a) Infrared image of polished device with a linear temperature scale.

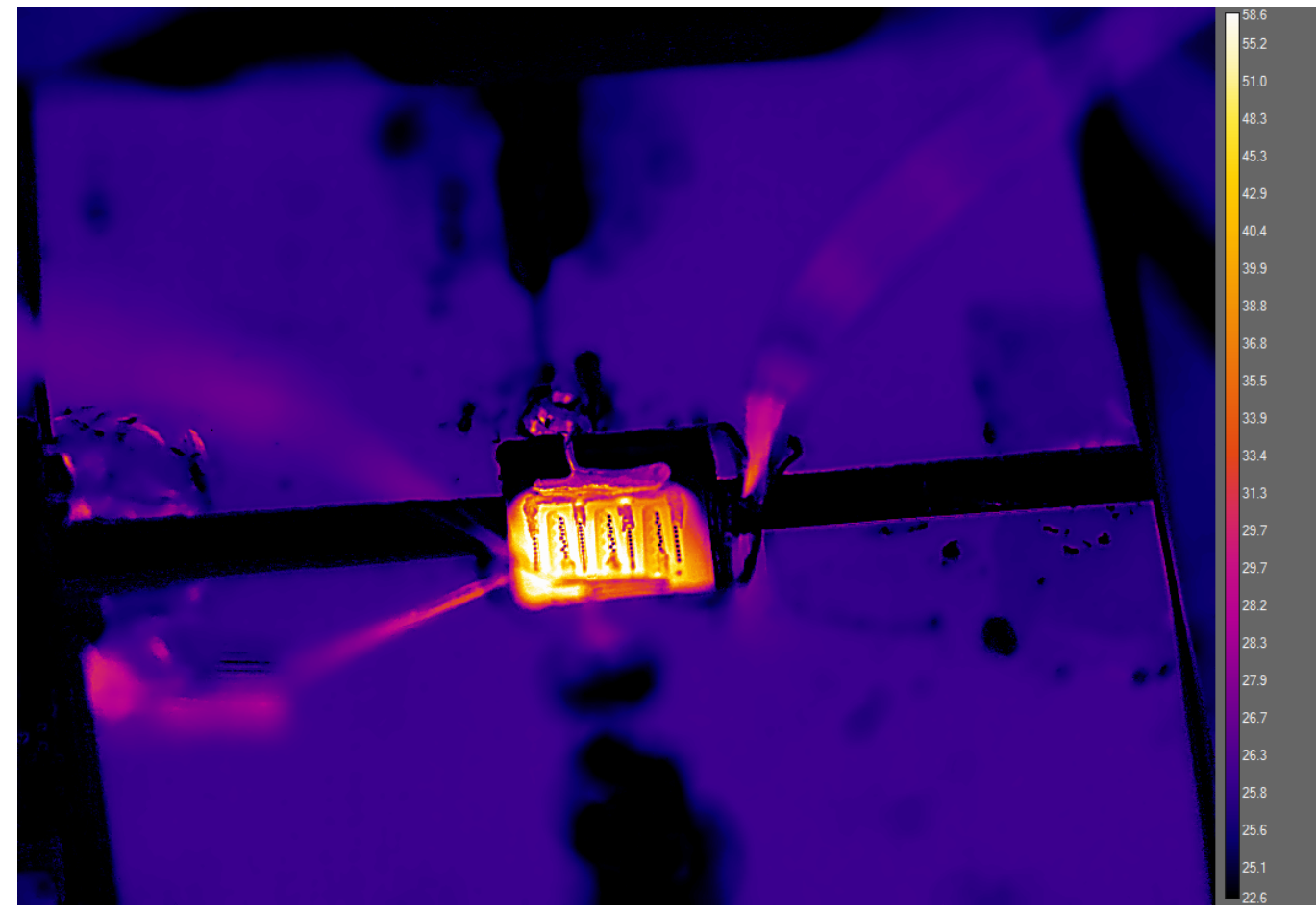

(b) Infrared image of polished device with Digital Detail Enhancement temperature scale [40].

Figure 3.28: Infrared imaging results of a polished test device shown with two different temperature scales (legend in units of ${ }^{\circ} \mathrm{C}$ ). 


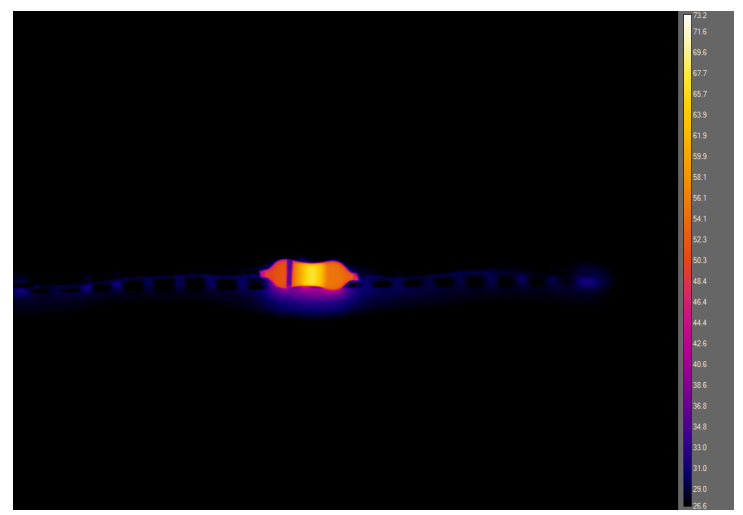

(a) Infrared image of powered resistor - Uncovered.

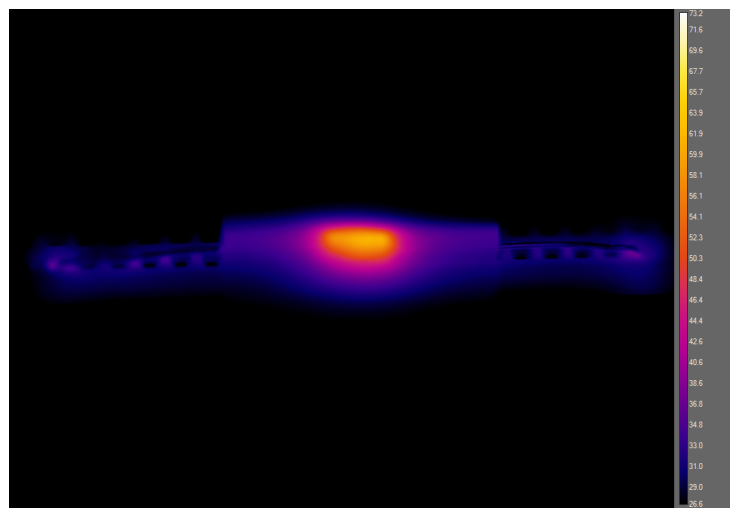

(b) Infrared image of powered resistor - Kapton covered.

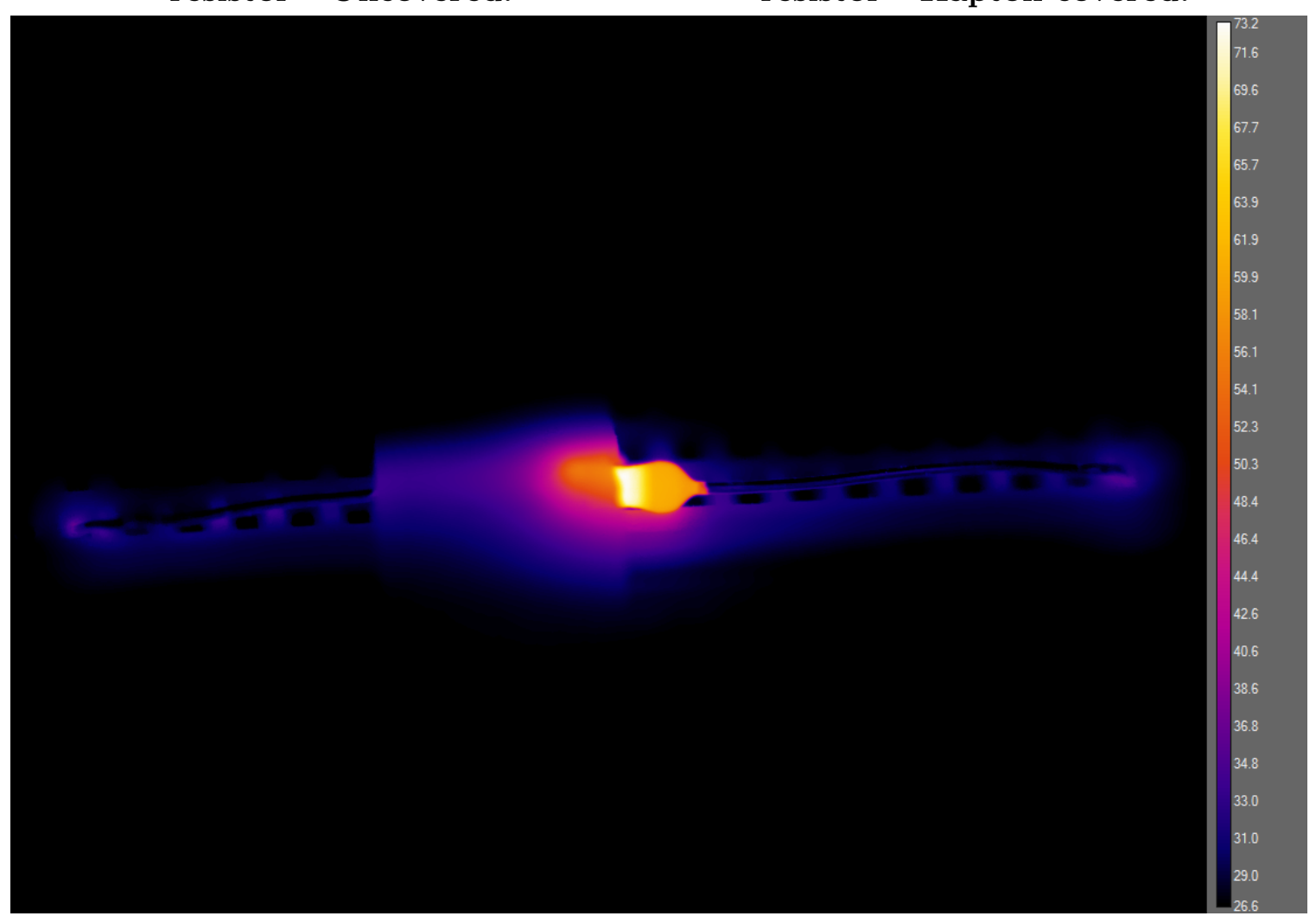

(c) Infrared image of powered resistor - Half covered.

Figure 3.29: Infrared imaging test of polyimide (Kapton) using a powered resistor to illustrate the effect of polyimide on the thermal imaging of the polished device (legend in units of ${ }^{\circ} \mathrm{C}$ ). 


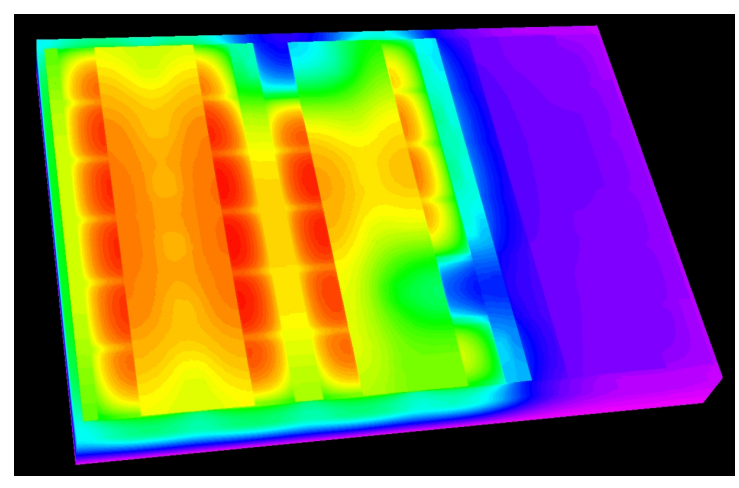

(a) Atar thermal approximation of failed polished device test.

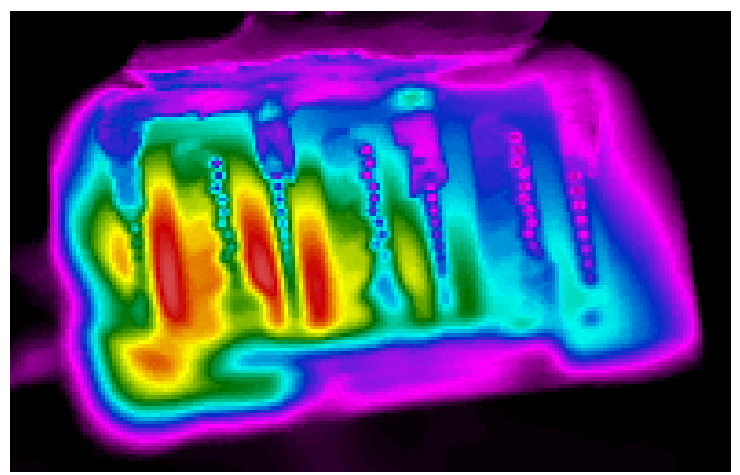

(b) Infrared image of polished device, colour-matched to the Atar scale.

Figure 3.30: Approximate thermal comparison of the Atar output and the thermal image of the powered polished device.

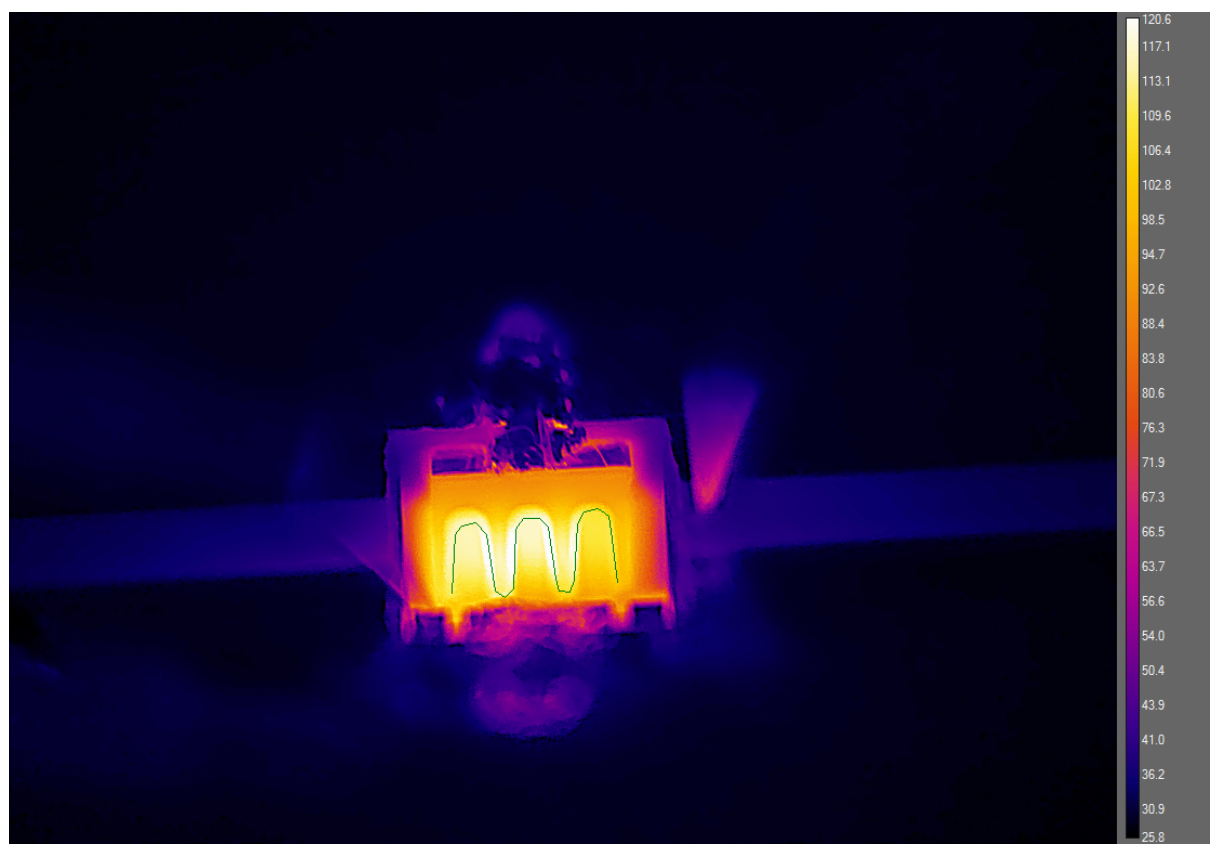

\begin{tabular}{|l|r|}
\hline Statistic [units] & Line 1 \\
\hline Mean ["C] & 112.7 \\
\hline Std. Dev. ["C] & 4.9 \\
\hline Center ["C] & $(484.5,498.0) 113.7$ \\
\hline Maximum ["C] & $(466,493) 120.2$ \\
\hline Minimum ["C] & $(561,525) 99.9$ \\
\hline Number of Pixels & 471 \\
\hline
\end{tabular}

Figure 3.31: Thermal image of the unpolished device and the extraction of the maximum and average $\mathrm{T}_{\mathrm{J}}$ (legend in units of ${ }^{\circ} \mathrm{C}$ ). 
Table 3.5: Comparison between measurements and simulation for electrothermal behaviour.

\begin{tabular}{|l||c|c|l|l|}
\hline & Measured & Simulated & $\begin{array}{l}\text { Simulation } \\
\text { Parameter }\end{array}$ & $\begin{array}{l}\text { Percent } \\
\text { Error }\end{array}$ \\
\hline $\mathrm{V}_{\mathrm{G}}(\mathrm{V})$ & 2 & 1.83 & Specified & $8.5 \%$ \\
\hline $\mathrm{V}_{\mathrm{D}}(\mathrm{V})$ & 11.25 & 11.25 & Specified & $0 \%$ \\
\hline $\mathrm{T}_{\mathrm{C}}\left({ }^{\circ} \mathrm{C}\right)$ & 111.6 & 110 & Specified & $1.434 \%$ \\
\hline $\mathrm{I}_{\mathrm{D}}(\mathrm{A})$ & 2.65 & 2.66 & V1:Iout & $0.377 \%$ \\
\hline $\mathrm{P}_{\mathrm{D}}(\mathrm{W})$ & 29.813 & 29.925 & $\begin{array}{l}\text { Sum of } \\
\text { Pown:I }\end{array}$ & $0.376 \%$ \\
\hline Max $\mathrm{T}_{\mathrm{J}}\left({ }^{\circ} \mathrm{C}\right)$ & 120.25 & 118.66 & M1:Max V & $1.322 \%$ \\
\hline Avg $\mathrm{T}_{\mathrm{J}}\left({ }^{\circ} \mathrm{C}\right)$ & 112.72 & 117.37 & $\begin{array}{l}\text { Average of } \\
\text { M1:Port Vs }\end{array}$ & $4.125 \%$ \\
\hline
\end{tabular}




\section{Chapter 4}

\section{Electrothermal Simulation Results}

With the electrical, thermal, and electrothermal simulations having all been independently verified as producing valid results, the full electrothermal simulations were run to gather data for different cases of deactivated cells. Some of the situations investigated include the location of the highest temperature cell, the $\Delta \mathrm{T}$ between the highest temperature and the lowest temperature (both lowest temperature active and lowest inactive cell), the maximum cell temperature as the number of deactivated cells increases, and a look into how the device behaves for extreme cases of deactivated cell clustering. While there are many other possible tests that could be run and statistics that could be analyzed, the situations presented give a good overview of the capability of the electrothermal simulations.

\subsection{Electrothermal Simulation Setup}

To examine the effects that deactivating cells has on the device performance, four different cases were considered. These cases were when one cell, two cells, three cells, and four cells were deactivated concurrently. For each case, random but unique combinations of cells were deactivated and information on cell currents, cell temperatures, cell powers, chip current, and chip power was collected and analyzed. The backside 
temperature was fixed at $25^{\circ} \mathrm{C}$, the drain voltage was set to 10 volts, and the gate voltage was adjusted to keep a constant power dissipation of around 100 Watts for all tests to track the effects of deactivating cells for a fixed power dissipation. The $25^{\circ} \mathrm{C}$ backside temperature and $100 \mathrm{~W}$ power dissipation were chosen to match the thermal simulations provided by GaN Systems as outline earlier in Section 3.1.3. The number of possible unique combinations for each case is detailed in Table 4.1. Since each simulation takes roughly 3 minutes to run, all 36 unique cases of one cell deactivated were run but only 200 random combinations for each of the two, three, and four cells deactivated cases were considered as the sample size.

Table 4.1: Number of unique combinations for each case considered and number of tests run.

\begin{tabular}{|l||c|c|}
\hline Case & Unique Combinations & No. Of Tests Run \\
\hline One Cell Deactivated & $\frac{36 !}{(36-35) ! \cdot 35 !}=36$ & 36 \\
\hline Two Cells Deactivated & $\frac{36 !}{(36-34) ! \cdot 34 !}=630$ & 200 \\
\hline Three Cells Deactivated & $\frac{36 !}{(36-33) ! \cdot 33 !}=7,140$ & 200 \\
\hline Four Cells Deactivated & $\frac{36 !}{(36-32) ! \cdot 32 !}=58,905$ & 200 \\
\hline
\end{tabular}

\subsection{Location of Highest Temperature Cell}

The location of the highest temperature cell naturally lies in the middle of the chip (cells 15, 16, 21, and 22) for the case where all cells are active. As more cells are deactivated, the location of the highest temperature has an increasingly higher probability of being in the region directly to the left and right of the middle cells $(9,10$, 27 , and 28) and slightly increased probability of being above or below the middle rows $(8,11,14,17,20,23,26$, and 29). These results are presented in Figure 4.1, which shows the distribution probability histograms for the location of the highest temperature cell as an increasing number of cells are deactivated. The cell numbering scheme and column colouring scheme is shown in Figure 4.2. A graphical depiction 
of the movement of the highest temperature cell location is illustrated in Figure 4.3.

\section{3 $\Delta \mathrm{T}$ of Highest and Lowest Temperature on Chip}

This set of analyses deals with the temperature gradients between the hottest and coolest cell (both active and inactive). The gradient is larger for the inactive cell cases as the inactive cell is closer to the backside temperature while all the active cells should be within a small range of each other. These distributions have been fit with a normal fit to observe how well the temperature gradient is normally distributed. The temperature gradient distributions for the $\Delta \mathrm{T}$ between the highest temperature cell and the lowest temperature inactive cell is shown in Figure 4.4 and the temperature gradient distributions for the $\Delta \mathrm{T}$ between the highest temperature cell and the lowest temperature active cell is shown in Figure 4.5.

\subsection{Maximum Cell Temperature Versus Number of Deactivated Cells}

The plot of maximum cell temperature as a function of the number of cells that have been deactivated as seen in Figure 4.6 shows that as the number of deactivated cells increases, the spread of the maximum temperature increases. This appears to mean that which cells are deactivated in relation to each other may have a larger effect on the maximum temperature of the cells. This will be explored more in the next section when analyzing some extreme cases of clustering of four deactivated cells. 


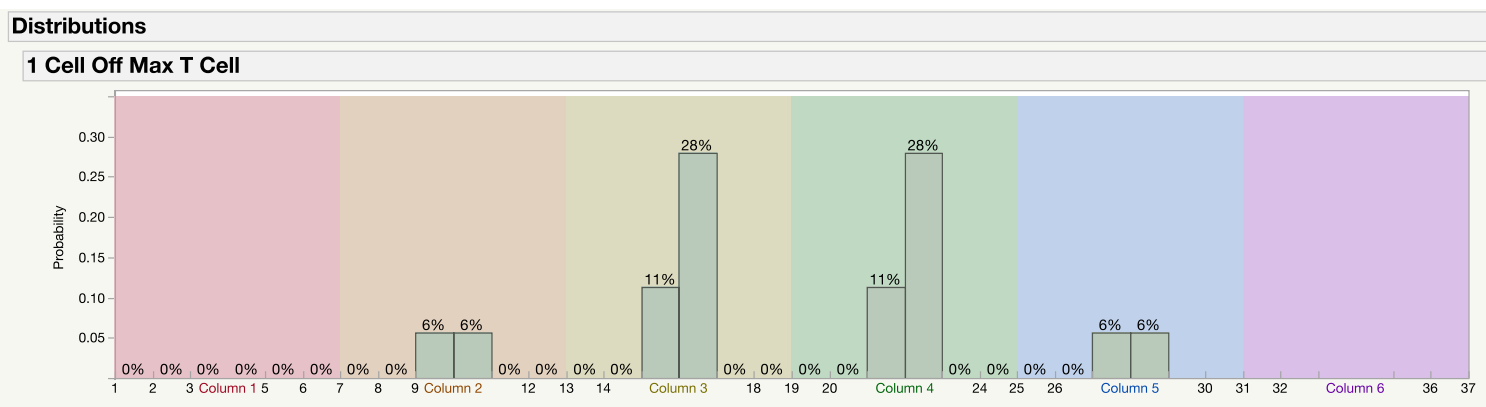

2 Cell Off Max T Cell

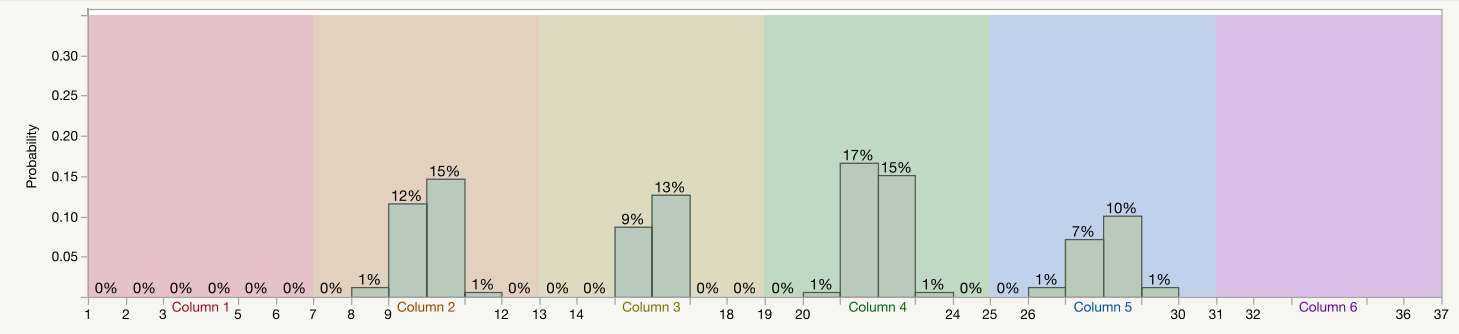

3 Cell Off Max T Cell

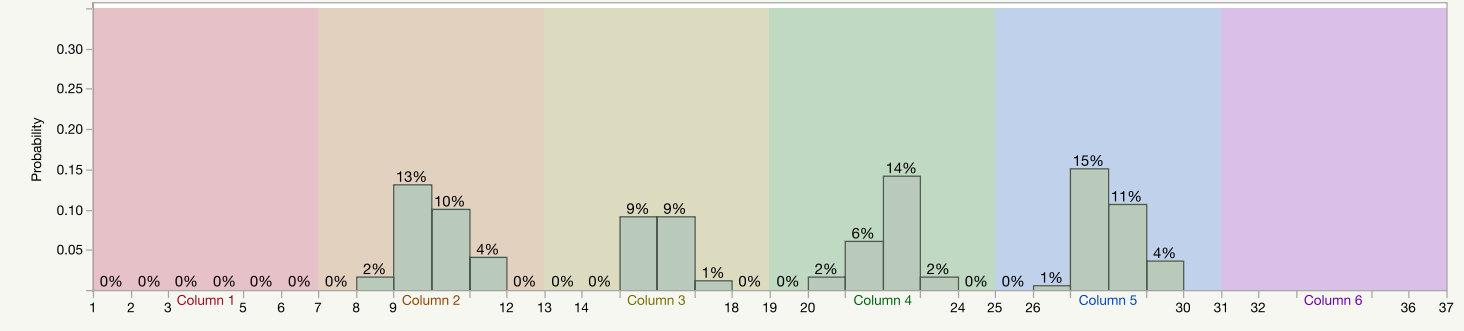

4 Cell Off Max T Cell

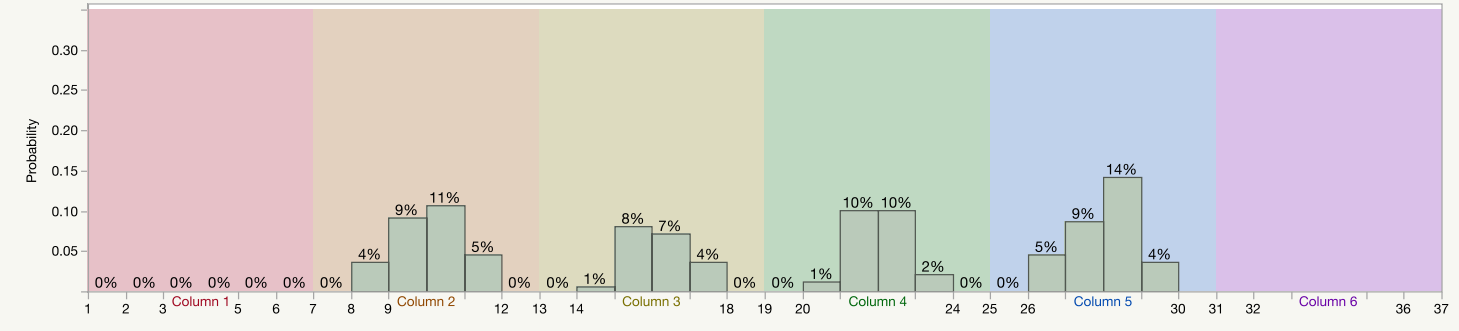

Figure 4.1: The maximum temperature cell location probability distribution for an increasing number of deactivated cells. 


\begin{tabular}{|c|c|c|c|c|c|}
\hline 6 & 12 & 18 & 24 & 30 & 36 \\
\hline 5 & 11 & 17 & 23 & 29 & 35 \\
\hline 4 & 10 & 16 & 22 & 28 & 34 \\
\hline 3 & 9 & 15 & 21 & 27 & 33 \\
\hline 2 & \begin{tabular}{|l|}
8 \\
\end{tabular} & 14 & 20 & 26 & 32 \\
\hline 1 & \begin{tabular}{|l|}
7 \\
\end{tabular} & 13 & 19 & 25 & 31 \\
\hline
\end{tabular}

Figure 4.2: Reference cell number and colour scheme for the probability distribution in Figure 4.1

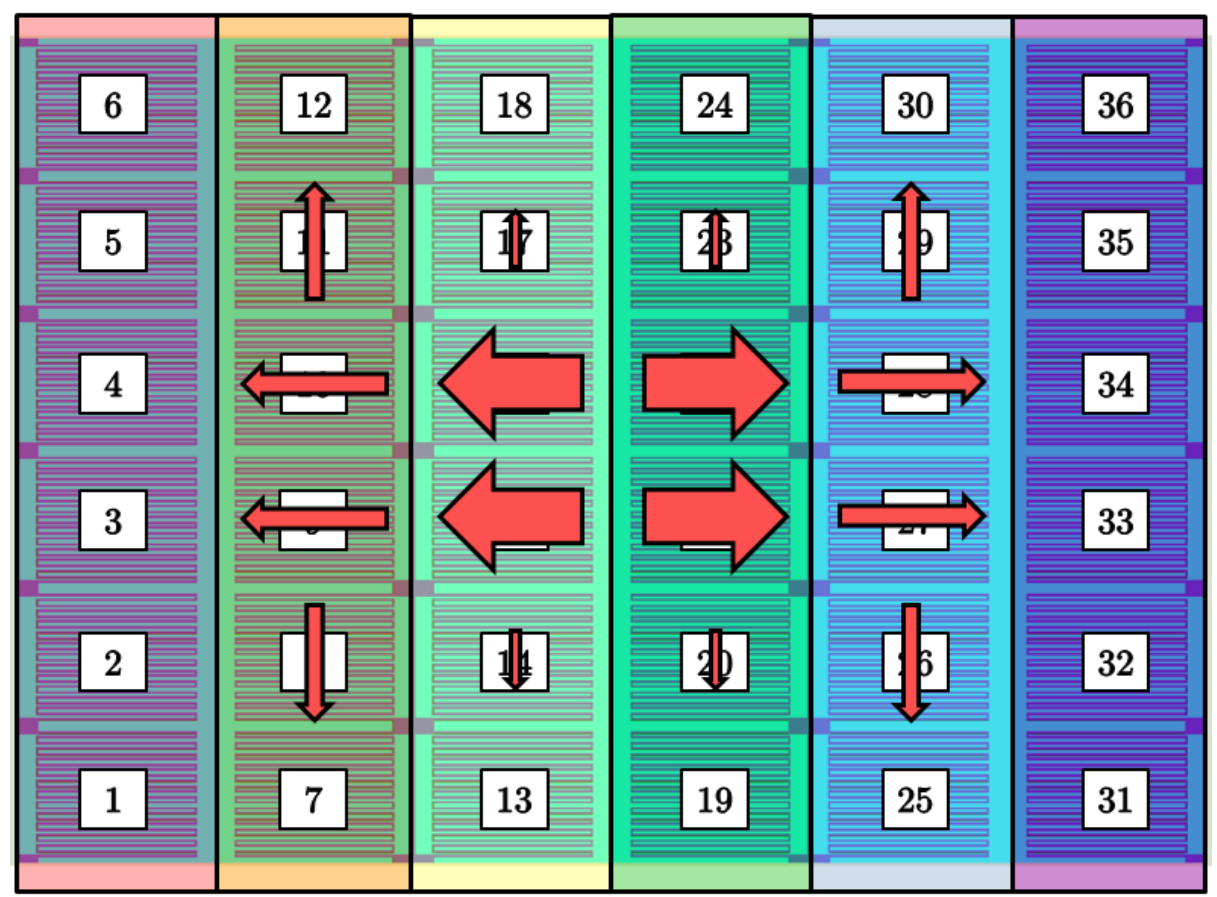

Figure 4.3: Depiction of the highest temperature cell movement with an increasing number of deactivated cells. 


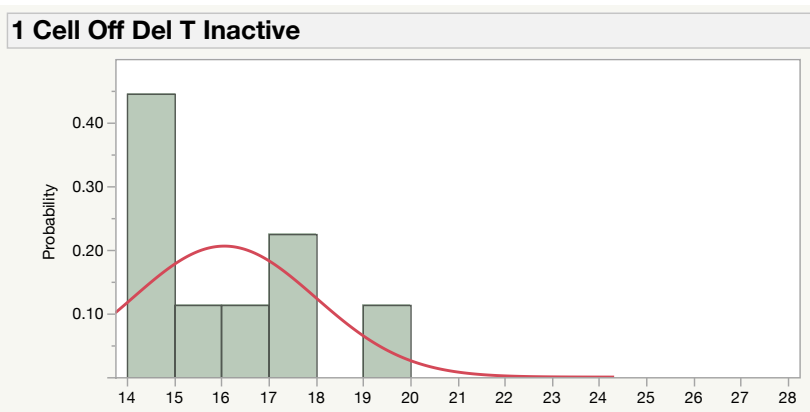

\section{Summary Statistics}

Mean $\quad 16.061756$

Std Dev $\quad 1.9376756$

$\mathrm{N}$

Variance

Range

Interquartile Range 5.7011705

3.7545869
5.7011705

3.4692711
Fitted Normal

Parameter Estimates

Type Parameter Estimate

Location $\mu \quad 16.061756$

Dispersion $\sigma \quad 1.9376756$

Normal(16.0618,1.93768)

2 Cell Off Del T Inactive

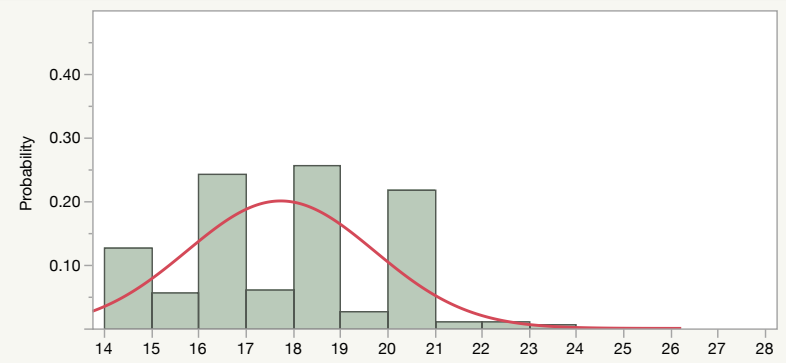

Summary Statistics

Mean $\quad 17.742781$ Std Dev $\quad 1.9913087$

Variance

Range

9.577343
Fitted Normal

Parameter Estimates

Type Parameter Estimate $\begin{array}{ll}\text { Location } \mu & 17.74278\end{array}$ $\begin{array}{ll}\text { Dispersion } \sigma & 1.9913087\end{array}$

Normal $(17.7428,1.99131)$

\section{Cell Off Del T Inactive}

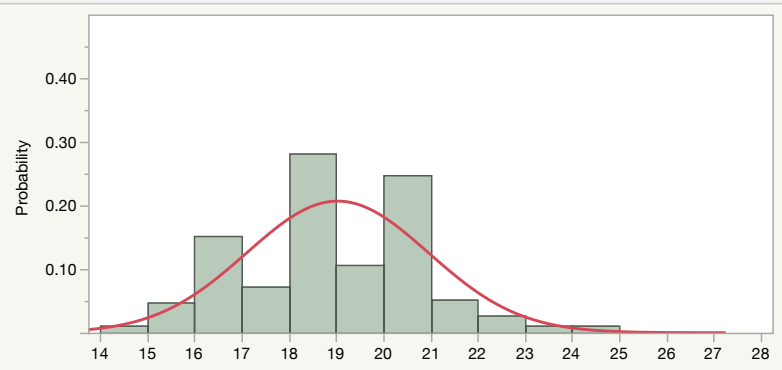

Normal(19.036,1.92903)

4 Cell Off Del T Inactive

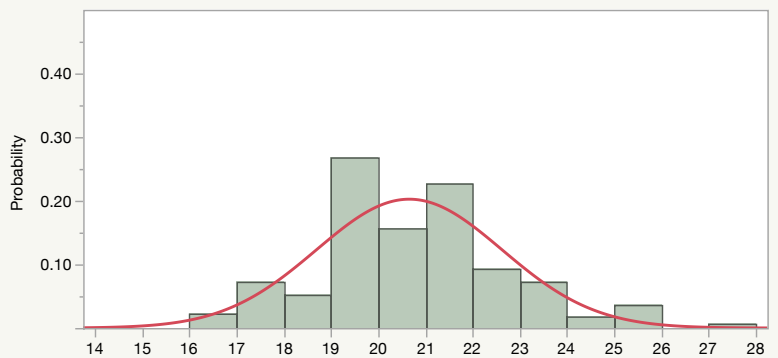

- Normal(20.6602,1.97005)

\section{Summary Statistics}

Mean

Std Dev

$\mathrm{N}$

Variance

Variance
Range

Interquartile Ran
19.035979

1.9290318

200
3.7211636 3.7211636 .7363265 3.472231
Fitted Normal

Parameter Estimates

Type Parameter Estimate

Location $\mu \quad 19.035979$

Dispersion $\sigma \quad 1.9290318$

Figure 4.4: Temperature gradient distributions for an increasing amount of deactivated cells relative to the lowest temperature inactive cell. Units for the bins (x-axis) are in ${ }^{\circ} \mathrm{C}$. 


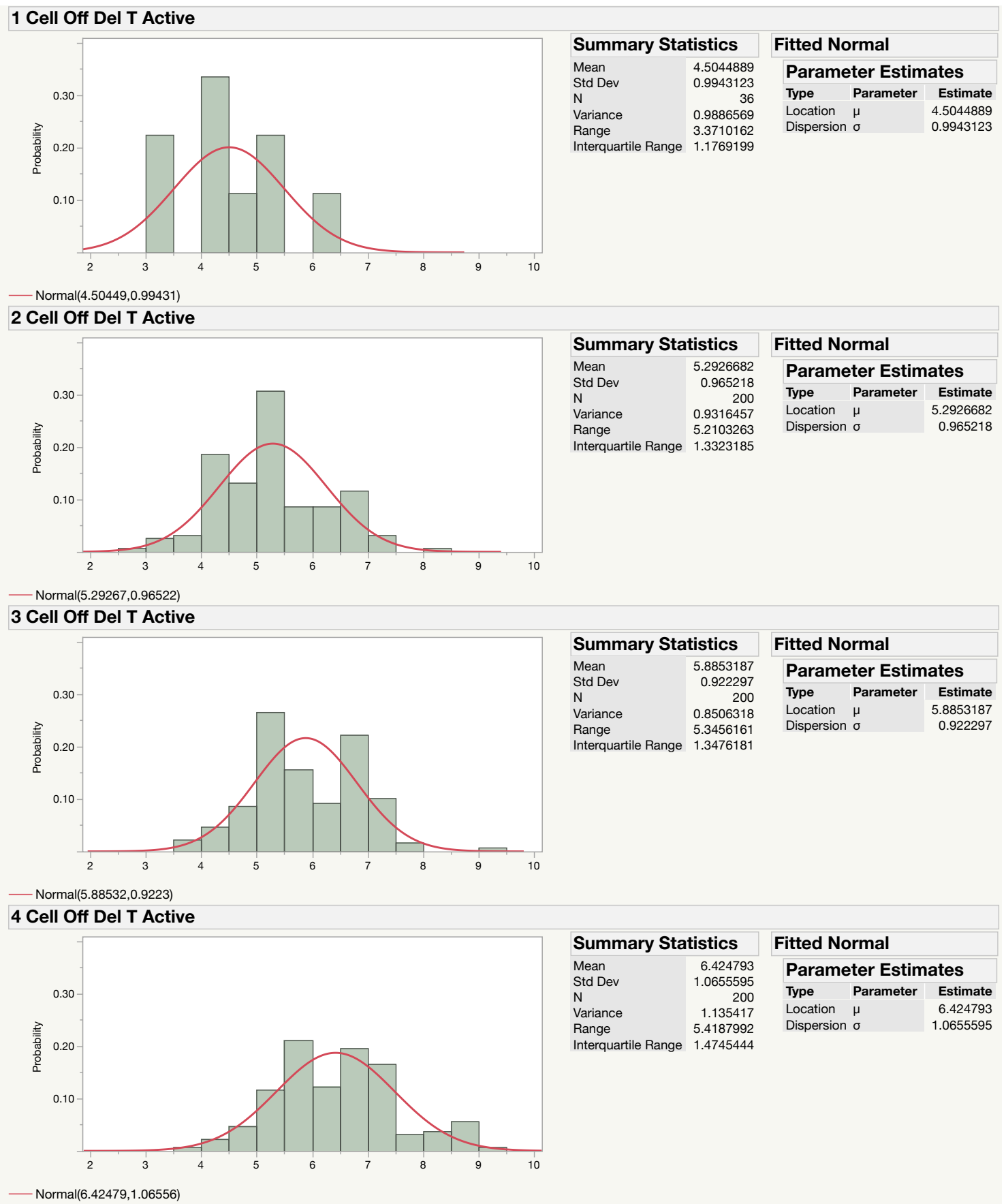

Figure 4.5: Temperature gradient distributions for an increasing amount of deactivated cells relative to the lowest temperature active cell. Units for the bins (x-axis) are in ${ }^{\circ} \mathrm{C}$. 


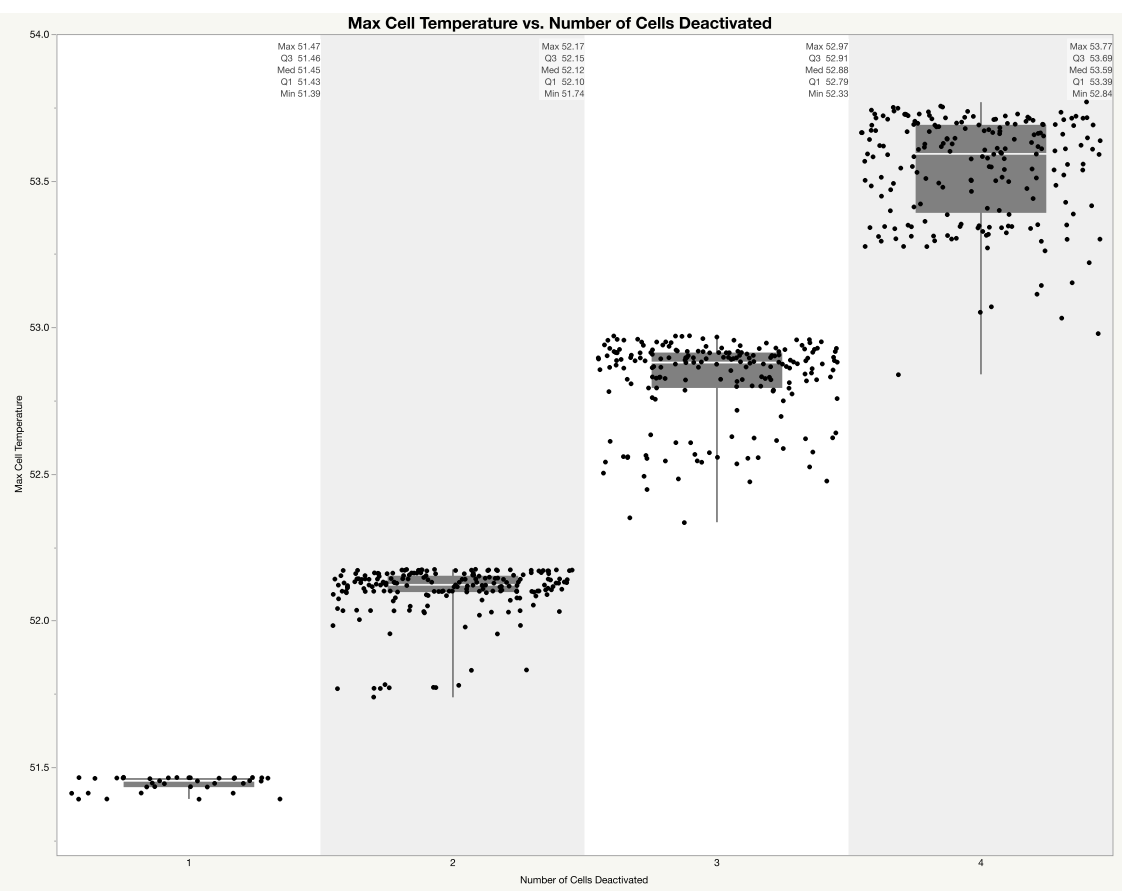

Figure 4.6: Depiction of the maximum cell temperature increasing and range spreading as the number of deactivated cells increases. Units for the maximum cell temperature $\left(\mathrm{y}\right.$-axis) are in ${ }^{\circ} \mathrm{C}$.

\subsection{Extreme Cases for Four Deactivated Cells}

This section explores some specific extreme cases of four deactivated cells. These cases represent spread out deactivations and clustering in the hottest and coolest spots on the chip to examine the effects. The results are presented in Table 4.2 and the highest and lowest values for each parameter are marked in red and blue respectively. From this it seems that the worst case would be if all the deactivated cells clustered in the corners. This case produced the highest temperatures and largest $\Delta \mathrm{T}$. 
Table 4.2: Results for several extreme cases of four deactivated cells.

\begin{tabular}{|c|c|c|c|c|c|c|c|}
\hline \multicolumn{6}{|c|}{ Cell Distribution } & Parameter & Value \\
\hline \multirow{2}{*}{ 西 } & \multirow[b]{2}{*}{ 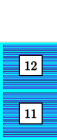 } & \multirow[b]{2}{*}{ 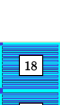 } & \multirow[b]{2}{*}{24} & \multirow[b]{2}{*}{ 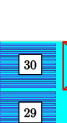 } & \multirow[b]{2}{*}{ 象 } & Max Temp (Orange Cell) $\left({ }^{\circ} \mathrm{C}\right)$ & 53.756 \\
\hline & & & & & & Min Active Cell Temp (Blue Cell) $\left({ }^{\circ} \mathrm{C}\right)$ & 49.01 \\
\hline 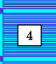 & 10 & 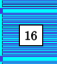 & 2 & 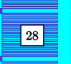 & 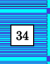 & Min Deactivated Cell Temp $\left({ }^{\circ} \mathrm{C}\right)$ & 32.27 \\
\hline \multirow{2}{*}{ 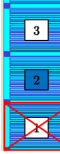 } & \multirow{2}{*}{ 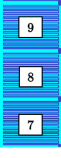 } & \multirow{2}{*}{13} & \multirow{2}{*}{21} & \multirow{2}{*}{27} & \multirow{2}{*}{ 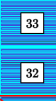 } & $\Delta \mathrm{T}$ High to Low Active $\left({ }^{\circ} \mathrm{C}\right)$ & 4.75 \\
\hline & & & & & & $\Delta \mathrm{T}$ High to Low Total $\left({ }^{\circ} \mathrm{C}\right)$ & 21.49 \\
\hline & & & 19. & & 象 & Chip Power $(\mathrm{W})$ & 99.55 \\
\hline \multirow[b]{2}{*}{6} & \multirow[b]{2}{*}{12} & \multirow{2}{*}{ 18 } & \multirow[b]{2}{*}{24} & \multirow[b]{2}{*}{ 䢗 } & \multirow[b]{2}{*}{36} & Max Temp (Orange Cell) $\left({ }^{\circ} \mathrm{C}\right)$ & 53.05 \\
\hline & & & & & & Min Active Cell Temp (Blue Cell) $\left({ }^{\circ} \mathrm{C}\right)$ & 47.95 \\
\hline$\overline{\underline{t}}$ & 10 & 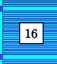 & 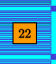 & 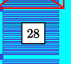 & 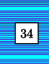 & Min Deactivated Cell Temp $\left({ }^{\circ} \mathrm{C}\right)$ & 37.99 \\
\hline \multirow{3}{*}{2} & 9 & \multirow{2}{*}{ 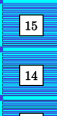 } & 21 & \multirow{2}{*}{27} & \multirow{3}{*}{ 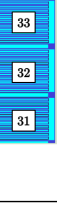 } & $\Delta \mathrm{T}$ High to Low Active $\left({ }^{\circ} \mathrm{C}\right)$ & 5.10 \\
\hline & 7 & & 20 & & & $\Delta \mathrm{T}$ High to Low Total $\left({ }^{\circ} \mathrm{C}\right)$ & 15.06 \\
\hline & & & & & & Chip Power $(\mathrm{W})$ & 99.88 \\
\hline & & & & & & Max Temp (Orange Cell) $\left({ }^{\circ} \mathrm{C}\right)$ & 52.83 \\
\hline 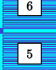 & 111 & 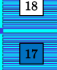 & 243 & 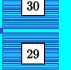 & 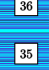 & Min Active Cell Temp (Blue Cell) $\left({ }^{\circ} \mathrm{C}\right)$ & 49.41 \\
\hline$\underline{\underline{\underline{4}}}$ & 10 & 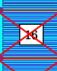 & 2 & 28 & 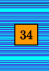 & Min Deactivated Cell Temp $\left({ }^{\circ} \mathrm{C}\right)$ & 33.10 \\
\hline 3 & 9 & 更 & 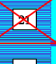 & 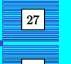 & 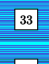 & $\Delta \mathrm{T}$ High to Low Active $\left({ }^{\circ} \mathrm{C}\right)$ & 3.42 \\
\hline 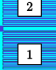 & 7 & 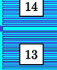 & 20 & 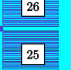 & 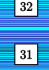 & $\Delta \mathrm{T}$ High to Low Total $\left({ }^{\circ} \mathrm{C}\right)$ & 19.73 \\
\hline & & & & & & Chip Power $(\mathrm{W})$ & 99.72 \\
\hline & & & & & & Max Temp (Orange Cell) $\left({ }^{\circ} \mathrm{C}\right)$ & 52.77 \\
\hline$\sqrt{5-16}$ & 11 & 178 & 23 & 290 & 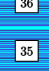 & Min Active Cell Temp (Blue Cell) $\left({ }^{\circ} \mathrm{C}\right)$ & 50.15 \\
\hline 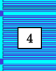 & 20 & 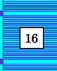 & 22 & 陮 & 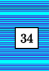 & Min Deactivated Cell Temp $\left({ }^{\circ} \mathrm{C}\right)$ & 35.20 \\
\hline 3 & 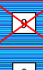 & 15 & 21 & 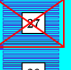 & 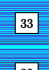 & $\Delta \mathrm{T}$ High to Low Active $\left({ }^{\circ} \mathrm{C}\right)$ & 2.63 \\
\hline$\sqrt{2+2}$ & 8 & 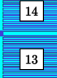 & 19 & 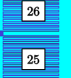 & 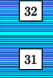 & $\Delta \mathrm{T}$ High to Low Total $\left({ }^{\circ} \mathrm{C}\right)$ & 17.58 \\
\hline & & & & & & Chip Power $(\mathrm{W})$ & 99.79 \\
\hline & & & & & & Max Temp (Orange Cell) $\left({ }^{\circ} \mathrm{C}\right)$ & 53.757 \\
\hline 望 & 11 & 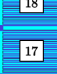 & 23 & 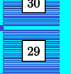 & 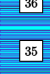 & Min Active Cell Temp (Blue Cell) $\left({ }^{\circ} \mathrm{C}\right)$ & 48.20 \\
\hline 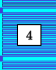 & 10 & 16 & 22 & 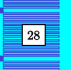 & 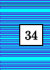 & Min Deactivated Cell Temp $\left({ }^{\circ} \mathrm{C}\right)$ & 25.89 \\
\hline 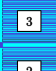 & 9 & 15 & 21 & 27 & 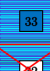 & $\Delta \mathrm{T}$ High to Low Active $\left({ }^{\circ} \mathrm{C}\right)$ & 5.55 \\
\hline 2 & 8 & 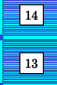 & 20 & 难 & 亟 & $\Delta \mathrm{T}$ High to Low Total $\left({ }^{\circ} \mathrm{C}\right)$ & 27.86 \\
\hline & & & & & & Chip Power (W) & 99.50 \\
\hline
\end{tabular}




\section{Chapter 5}

\section{Conclusions}

\subsection{Summary}

The objective of this thesis was to explore the electrothermal behaviour of an array of parallel transistors on a single chip as a number of cells were deactivated. This was done by coupling created Atar thermal models with a given SPICE electrical model and running relevant tests.

First, a background of the pertinent theory behind the semiconductor devices, thermodynamics, thermal effects in GaN, and the simulations important to the thesis was presented. Following this was a description of how the simulations were put together and verified before describing the results of the simulations and offering a preliminary analysis of said results.

\subsection{Thesis Contributions}

There were three major contributions to the objective outlined in the beginning of this thesis:

- Designed and modelled a three dimensional thermal simulation of a parallel array gallium nitride eHEMT device using Atar, adapted electrical models 
from LTSpice into OptiSPICE, coupled the thermal models from Atar into OptiSPICE to provide linked electrothermal simulation capabilities.

- Independently verified the operation of each simulation (electrical, thermal, and electrothermal) using models, previously completed simulations, and physical testing data. This was done to ensure the simulations results were sensible at every step of the process.

- Performed electrothermal simulations for a variety of cases to determine the effects that deactivating cells had on the device behaviour and made a basic preliminary analysis of some of the resulting data.

\subsection{Future Work}

Setting the simulators up for running electrothermal simulations and examining the behaviour of the devices for differing numbers and locations of cells active on the chip was just the first step towards the future goal of examining the impact of direct thermal and temperature distribution effects for actual device applications. Modifying the results of this thesis to perform transient simulations will allow for the investigation into the effects on RF and power device operation which may lead to the enhancement of device yield.

By examining the thermal and electrical characteristics of devices with nonyielding cells, it may be possible to determine what, if any, other cells should be disabled in order to maintain proper device operation and lifetime by limiting the effects of hot spots and large thermal gradients. In-depth analysis may lead to the creation of adaptive algorithms that could determine the best cell distribution to maximize performance and lifetime based on information gathered after identifying defective islands through electrical testing following fabrication. This information 
could then be used to dictate where interconnections are formed to isolate defective islands along with the algorithmically determined cells, enhancing the yield of functional devices that may have not been able to be sold otherwise. 


\section{References}

[1] G. P. Klowak, A. Mizan, and J. Roberts, "Embedded packaging for devices and systems comprising lateral gan power transistors", pat. US 2016/0240471 A1, Aug. 18, 2016.

[2] A. Lidow, J. Strydom, M. de Rooij, and D. Reusch, GaN Transistors for Efficient Power Conversion (2nd Edition), 2nd. Wiley, 2015, ISBN: 9781118844762.

[3] A. Lidow. (2011). Is it the End of the Road for Silicon in Power Conversion?, Application Note: AN001, Efficient Power Conversion Corporation, [Online]. Available: http : / / epc-co . com / epc / Portals / 0 / epc / documents / product training/Appnote_Si_endofroad.pdf.

[4] S. Taranovich, "Power Si vs. GaN vs. SiC: Which process and supplier are best for my design?", EDN, 3 Mar. 1, 2013.

[5] U. K. Mishra, L. Shen, T. E. Kazior, and Y.-F. Wu, "GaN-Based RF Power Devices and Amplifiers", in Proceedings of the IEEE, Vol. 96, No. 2, 2008.

[6] G. P. Klowak, C. McKnight-MacNeil, H. Tweddle, A. Mizan, and N. Springett, "Fault tolerant design for large area nitride semiconductor devices", pat. US 2015/0162252 A1, Jun. 11, 2015.

[7] GaN Systems. (2018). GS66516T | GaN Systems, [Online]. Available: https: //gansystems.com/gan-transistors/gs66516t/. 
[8] R. F. Pierret, Semiconductor Fundamentals: Volume I (2nd Edition), ser. Modular Series on Solid State Devices. Addison-Wesley, 1988, vol. I, ISBN: 0201122952.

[9] R. Nave. (1998). Fermi level and fermi function, [Online]. Available: http:// hyperphysics.phy-astr.gsu.edu/hbase/Solids/Fermi.html.

[10] S. O. Kasap, Principles of Electronic Materials and Devices, Third. McGrawHill, 2006, ISBN: 0072957913.

[11] G. W. Neudeck, The PN Junction Diode: Volume II (2nd Edition), ser. Modular Series on Solid State Devices. Addison-Wesley, 1989, vol. II, ISBN: 9780201122961.

[12] N. S. J. Murphy, F. Berz, and I. Flinn, "Carrier mobility in MOS transistors", Philips Technical Review, vol. 31, no. 7-9, pp. 237-244, 1970.

[13] A. Pérez-Tomás, M. Placidi, N. Baron, S. Chenot, Y. Cordier, J. Moreno, J. Millán, and P. Godignon, "2deg HEMT mobility vs inversion channel MOSFET mobility", Materials Science Forum, vol. 645-648, pp. 1207-1210, 2010. DOI: 10.4028/www.scientific.net/msf.645-648.1207.

[14] J. H. Davies, The Physics of Low-dimensional Semiconductors: An Introduction. Cambridge University Press, 1998, IsBN: 9780521484916.

[15] Z. Zhang and J. T. Yates, "Band bending in semiconductors: Chemical and physical consequences at surfaces and interfaces", Chemical Reviews, vol. 112, no. 10 , pp. 5520-5551, 2012. DOI: 10.1021/cr3000626.

[16] S. M. Sze and K. K. Ng, Physics of Semiconductor Devices. Wiley-Interscience, 2006, ISBN: 9780471143239.

[17] J. P. Ibbetson, P. T. Fini, K. D. Ness, S. P. DenBaars, J. S. Speck, and U. K. Mishra, "Polarization effects, surface states, and the source of electrons in AlGaN/GaN heterostructure field effect transistors", Applied Physics Letters, vol. 77 , no. 2 , pp. 250-252, 2000. DOI: 10.1063/1.126940. 
[18] X.-G. He, D.-G. Zhao, and D.-S. Jiang, "Formation of two-dimensional electron gas at $\mathrm{AlGaN} / \mathrm{GaN}$ heterostructure and the derivation of its sheet density expression", Chinese Physics B, vol. 24, no. 6, p. 067 301, 2015. DoI: 10.1088/16741056/24/6/067301.

[19] D. L. Pulfrey, Understanding Modern Transistors and Diodes. Cambridge University Press, 2010, ISBN: 9780521514606.

[20] A. Hamdoune, "Fabrication and DC Characterizations of AlGaN/GaN High Electron Mobility Transistors with Undoped and Doped Donor Layers", Romanian Journal of Physics, vol. 59, no. 1-2, pp. 155-162, 2014.

[21] M. N. Ozisik, Heat Conduction, 2nd Edition. Wiley-Interscience, 1993, ISBN: 0471532568 .

[22] A. Bejan and A. D. Kraus, Heat Transfer Handbook. Wiley-Interscience, 2003, ISBN: 0-471-39015-1.

[23] P. Chyurlia, "Thermal Analysis of AlGaN/GaN HEMT monolithic integration with CMOS on Silicon <111> Substrates", Master's thesis, Carleton University, 2006 .

[24] K. R. Bagnall, "Device-level thermal analysis of gan-based electronics", Master's thesis, Massachusetts Institute of Technology, 2013.

[25] T. Bechtold, E. B. Rudnyi, and J. G. Korvink, "Dynamic electro-thermal simulation of microsystems - a review", Journal of Micromechanics and Microengineering, vol. 15, no. 11, R17-R31, 2005. DOI: 10.1088/0960-1317/15/11/R01.

[26] J. E. Sergent and A. Krum, Thermal Management Handbook: For Electronic Assemblies. McGraw-Hill Education, 1998, ISBN: 0070266999. 
[27] M. K. Chattopadhyay and S. Tokekar, "Thermal model for dc characteristics of algan/gan hemts including self-heating effect and non-linear polarization", Microelectronics Journal, vol. 39, no. 10, pp. 1181-1188, 2008. DOI: 10.1016/j. mejo.2008.01.043.

[28] W. Tan, M. Uren, P. Fry, P. Houston, R. Balmer, and T. Martin, "High temperature performance of AlGaN/GaN HEMTs on si substrates", Solid-State Electronics, vol. 50, no. 3, pp. 511-513, 2006. DOI: 10.1016/j.sse.2006.02.008.

[29] D. Pandey, A. Bhattacharjee, and T. R. Lenka, "Study on temperature dependence scattering mechanisms and mobility effects in GaN and GaAs HEMTs", in Physics of Semiconductor Devices, Springer International Publishing, 2013, pp. 67-70. DOI: 10.1007/978-3-319-03002-9_15.

[30] U. Chowdhury, J. Jimenez, C. Lee, E. Beam, P. Saunier, T. Balistreri, S.-Y. Park, T. Lee, J. Wang, M. Kim, J. Joh, and J. del Alamo, "TEM observation of crack- and pit-shaped defects in electrically degraded GaN HEMTs", IEEE Electron Device Letters, vol. 29, no. 10, pp. 1098-1100, 2008. DoI: 10.1109/led. 2008.2003073.

[31] M. N. Gurusinghe, S. K. Davidsson, and T. G. Andersson, "Two-dimensional electron mobility limitation mechanisms in AlxGa1-xN/GaN heterostructures", Physical Review B, vol. 72, no. 4, 2005. DoI: 10.1103/physrevb.72.045316.

[32] M. Engelhardt. (2017). Spice differentiation | analog devices, [Online]. Available: http://www.analog.com/en/technical-articles/spice-differentiation.html.

[33] S. B. R. Devireddy and S. Biswas, "Effect of fiber geometry and representative volume element on elastic and thermal properties of unidirectional fiberreinforced composites", Journal of Composites, vol. 2014, pp. 1-12, 2014. DOI: $10.1155 / 2014 / 629175$. 
[34] T. Smy, D. Walkey, and S. Dew, "A 3d thermal simulation tool for integrated devices-atar", IEEE Transactions on Computer-Aided Design of Integrated Circuits and Systems, vol. 20, no. 1, pp. 105-115, 2001. DOI: 10.1109/43.905679.

[35] T. Smy, D. Walkey, and S. Dew, "Transient 3D heat flow analysis for integrated circuit devices using the transmission line matrix method on a quad tree mesh", Solid-State Electronics, vol. 45, no. 7, pp. 1137-1148, 2001. DOI: 10.1016/s00381101(01)00066-1.

[36] D. Celo, P. Gunupudi, R. Khazaka, D. Walkey, T. Smy, and M. Nakhla, "Fast simulation of steady-state temperature distributions in electronic components using multidimensional model reduction", IEEE Transactions on Components and Packaging Technologies, vol. 28, no. 1, pp. 70-79, 2005. DOI: 10.1109/tcapt. 2004.838877 .

[37] W. Liu and A. A. Balandin, "Thermal conduction in AlxGa1-xN alloys and thin films", Journal of Applied Physics, vol. 97, no. 7, p. 073710, 2005. DOI: 10.1063/1.1868876.

[38] T. Yamazaki, J. T. Asubar, H. Tokuda, and M. Kuzuhara, "Impact of rounded electrode corners on breakdown characteristics of AlGaN/GaN high-electron mobility transistors", Applied Physics Express, vol. 11, no. 5, p. 054 102, 2018. DOI: $10.7567 /$ apex.11.054102.

[39] P. Tsilingiris, "Comparative evaluation of the infrared transmission of polymer films", Energy Conversion and Management, vol. 44, no. 18, pp. 2839-2856, 2003. DOI: $10.1016 / \mathrm{s} 0196-8904(03) 00066-9$.

[40] N. Högasten and R. Lindner. (). Digital detail enhancement - technical note, FLIR Systems, [Online]. Available: https://www.flirmedia.com/MMC/CVS/ Tech_Notes/TN_0003_EN.pdf. 


\section{Appendix A}

\section{Model Files}

\section{A.1 GS66516T LTSpice Model}

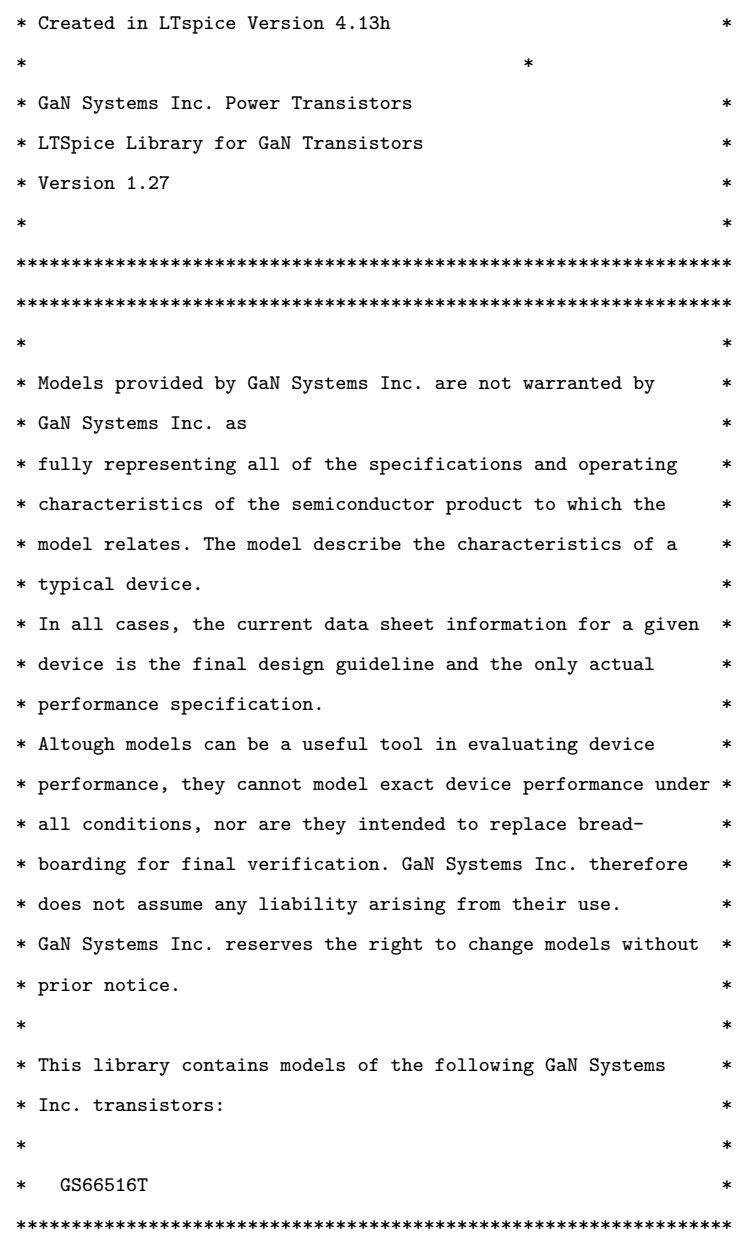

$* \$$

.subckt GS66516T gatein drainin sourcein T1 


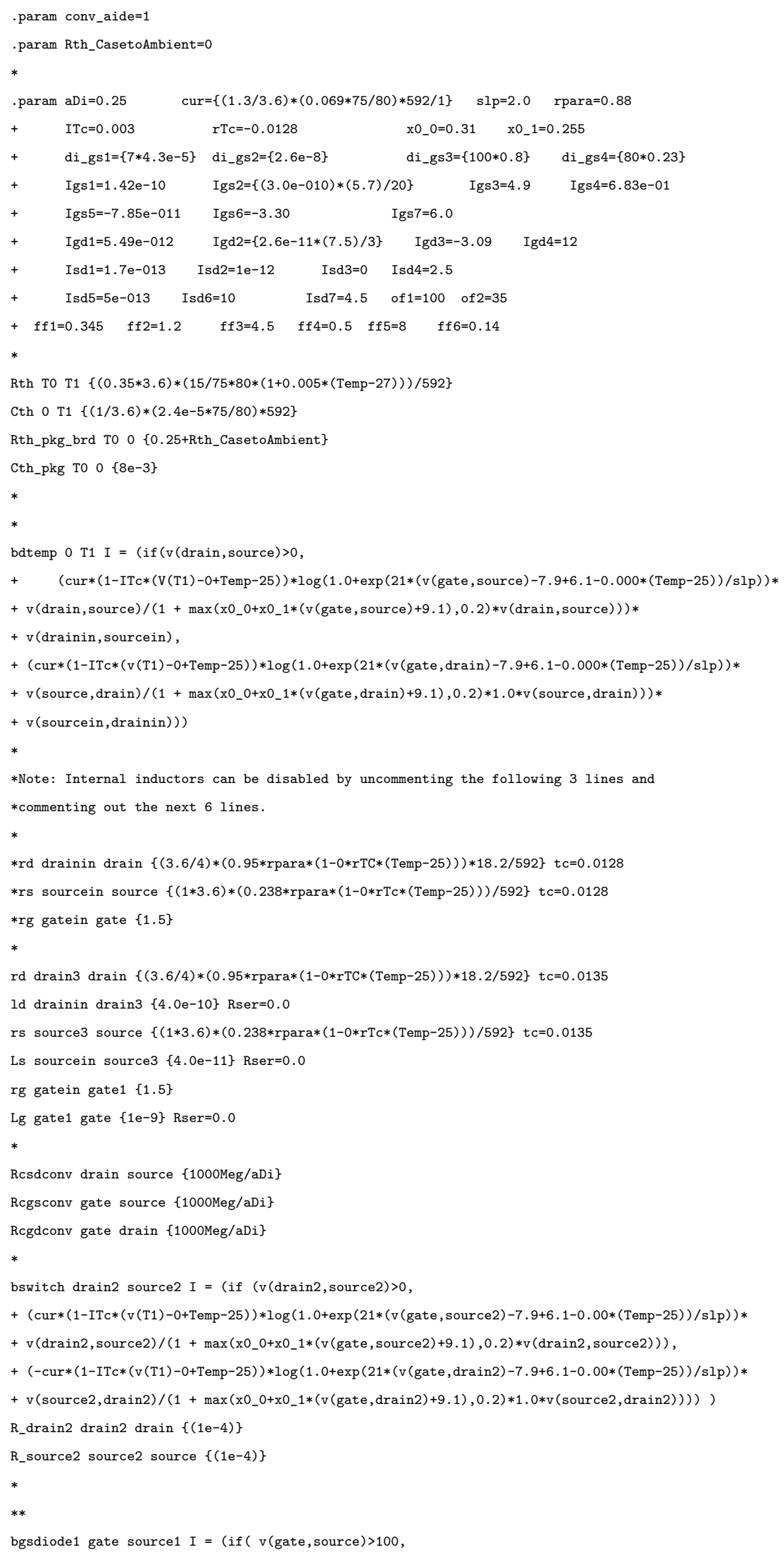


$+\left(0.2 *\left(1 *\right.\right.$ conv_aide $* 10.5 * a D i / 1077 *\left(d_{1}\right.$ gs $_{1} *\left(\exp \left(16 *(100.0) /\right.\right.$ di_gs $\left.\left._{3}\right)-1\right)+$ di_gs $2 *(\exp (16 *$

$+(100.0) / \mathrm{di}$ gs 4$)-1))) *(1+0.005 *($ Temp-27) $) *(1 / 3.6 * 592)) *(1+0.09 * \exp (0.051 *($ Temp-27) $))$,

$+\left(0.2 *\left(1 *\right.\right.$ conv_aide $* 10.5 * a D i / 1077 *\left(d_{1}\right.$ gs $1 *(\exp (16 *(\mathrm{v}($ gate, source 1$)) /$ di_gs 3$)-1)+$ di_gs $2 *$

$+\left(\exp \left(16 *\left(\mathrm{v}(\right.\right.\right.$ gate, source1) $\left.\left.\left.\left.\left.\left.\left.) / \mathrm{di} \_\mathrm{gs} 4\right)-1\right)\right)\right) *(1+0.005 *(\mathrm{Temp}-27))\right) *(1 / 3.6 * 592)\right) *(1+0.09 * \exp (0.051 *(\operatorname{Temp}-27)))\right)$

R_source1 source1 source $\{(14.47 / 380)\}$

$*$

**

bgddiode1 gate drain1 $\mathrm{I}=$ (if ( $\mathrm{v}$ (gate, drain) $>25$,

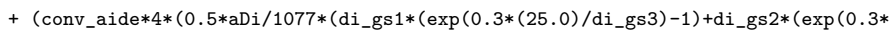

$+(25.0) /$ di_gs 4$)-1))) *(1+0.005 *($ Temp-27) $) *(0.2 / 3.6 * 592))$,

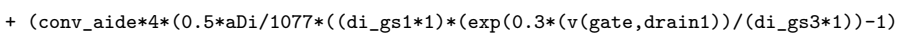

$++\left(\right.$ di_gs $\left._{2} *(1)\right) *\left(\exp \left(0.3 *(\mathrm{v}(\right.\right.$ gate, $\left.\left.\left.\left.\left.\left.\left.\operatorname{drain} 1)) /\left(\mathrm{di}_{\text {_gs }} 4 * 1\right)\right)-1\right)\right)\right) *(1+0.005 *(\operatorname{Temp}-27)) *(0.2 / 3.6 * 592)\right)\right)\right)$

R_drain1 drain1 drain $\{14.47 / 380\}$

*

bdsdiode1 drain1 source1 I $=($ if $(\mathrm{v}($ drain1, source1) $>850$,

$+\left(0.4 \mathrm{e}-9 *\right.$ conv_aide $* 200 *\left(0.5 * \mathrm{aDi} / 1077 *\left(\right.\right.$ di_gs $_{1} *(\exp (5 *(850.0-630+((\mathrm{Temp}-25) / 1.75)) /$ di_gs 3$)-1)+1 *$ di_gs $2 *(\exp (5 *$

$+(850.0-630+(($ Temp-25) $/ 1.75)) /$ di_gs 4$)-1))) *(1+0.1 *($ Temp-27) $) *(0.2 / 3.6 * 592))$

$+(0.4 \mathrm{e}-9 *$ conv_aide $* 200 *(0.5 * \mathrm{aDi} / 1077 *(($ di_gs $1 * 1) *(\exp (5 *(\mathrm{v}($ drain1, source 1$)-630+((\mathrm{Temp}-25) / 1.75)) /($ di_gs $3 * 1))-1)$

$++1 *($ di_gs $2 *(1)) *(\exp (5 *(\mathrm{v}($ drain1, source1) $-630+((\operatorname{Temp}-25) / 1.75)) /($ di_gs 41$))-1))) *(1+0.1 *(\operatorname{Temp}-27)) *(0.2 / 3.6 * 592))))$

$*$

bdsdiode2 drain1 source1 I $=($ if $(\mathrm{v}($ drain1, source 1$)>750$,

$+\left(0.1 \mathrm{e}-3 *\right.$ conv_aide $* 200 *\left(0.5 * a D i / 1077 *\left(d_{1}\right.\right.$ gs $1 *(\exp (0.5 *(750.0-670) /$ di_gs3 $\left.\left.))\right)\right)$

$+*(1+0.06 * \exp (0.1 *($ Temp -27$))) *(0.2 / 3.6 * 592))$,

$+\left(0.1 \mathrm{e}-3 *\right.$ conv_aide $* 200 *\left(0.5 * \mathrm{aDi} / 1077 *((\right.$ di_gs 1$))\left(\exp \left(0.5 *(\mathrm{v}(\right.\right.$ drain, source $)-670) /\left(\right.$ di_gs $\left.\left.\left.\left.\left._{3}\right)\right)\right)\right)\right)$

$+*(1+0.09 * \exp (0.1 *(\mathrm{Temp}-27))) *(0.2 / 3.6 * 592))))$

$*$

bgddiode2 gate drain1 I $=($ if $(\mathrm{v}$ (gate, drain 1$)>30$,

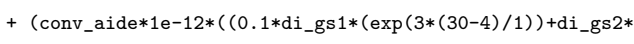

$+(\exp (3 *(30-4) / 1)))) *(1+0.005 *($ Temp-25) $)) *(1 / 3.6 * 592)$,

$+\left(\operatorname{conv} \_a i d e * 1 \mathrm{e}-12 *((0.1 *\right.$ di_gs $1 *(\exp (3 *(\mathrm{v}($ gate,drain 1$)-4) / 1))+$ di_gs $2 *$

$+(\exp (3 *(\mathrm{v}($ gate, $\operatorname{drain} 1)-4) / 1)))) *(1+0.005 *($ Temp-25) $)) *(1 / 3.6 * 592)))$

$* * * *$

$*$

C_GS gate source $\{(1.25 / 7 * \operatorname{Igs} 1 / 120 / 2.18) * 3.5 * 592\}$

C_GS1 gate source $Q=((1 / 7 * 10 / 120 / 2.18 * 1.5) * 592 * 1.5 *((0.5 * \operatorname{Igs} 2 * \operatorname{Igs} 4 * \log (1+\exp (f f 5 * 0.5 *(\mathrm{v}($ gate, source $)-\operatorname{Igs} 3+$

$+4.6) / 0.9933))-\operatorname{Igs} 5 * \operatorname{Igs} 7 * \log (1+\exp (f f 6 *(v($ source, $\operatorname{drain})-\operatorname{Igs} 6) / \operatorname{Igs} 7)))))$

*

C_GD gate drain $\{(0.8 / 17 * \operatorname{Igd} 1 / 30 / 2.18) * 1.5 * 592\}$

C_GD1 gate drain $Q=((0.7 / 7 * 1 / 25 / 2.18 * 0.8) * 592 *((0.5 * \operatorname{Igs} 2 * \operatorname{Igs} 4 * \log (1+\exp (f f 1 * 6 *(v($ gate, drain $)-\operatorname{Igs} 3+o f 1-50) /$

$+(\operatorname{Igs} 4 * f f 3)))+\operatorname{Igd} 2 * \operatorname{Igd} 4 * \log (1+\exp (0.5 * f f 2 *(v($ gate, drain $)-\operatorname{Igd} 3+o f 2-30) /(\operatorname{Igd} 4$

$+(* f(4)))))$ )

$*$

C_SD source drain $\{(2 / 7 *$ Isd $1 / 2.18) * 10 * 592\}$

C_SD1 source drain $Q=(1 / 7 * 1 / 2.18 * 18 * 592 *(4 * \operatorname{Isd} 2 * \operatorname{Isd} 4 * \log (1+\exp (0.1 *(v($ source,drain $)-\operatorname{Isd} 3+145) /$ Isd4 $))+$

$+\quad \operatorname{Isd} 5 * \operatorname{Isd} 7 * \log (1+\exp (1.5 *(\mathrm{v}($ source, drain) $-I s d 6+55) / I s d 7))$

$+\quad+5.7 * 0.0 *(0.5 * 2.5 \mathrm{e}-12 * 0.643 * \log (1+\exp (\mathrm{v}($ source, drain $)-4.68+80)) /(0.643$

$+* 3.5)))$ )

.ends

$* \$$ 


\section{Appendix B}

\section{Simulation Input Files}

\section{B.1 OptiSPICE Electrothermal Simulation Input Example Code}

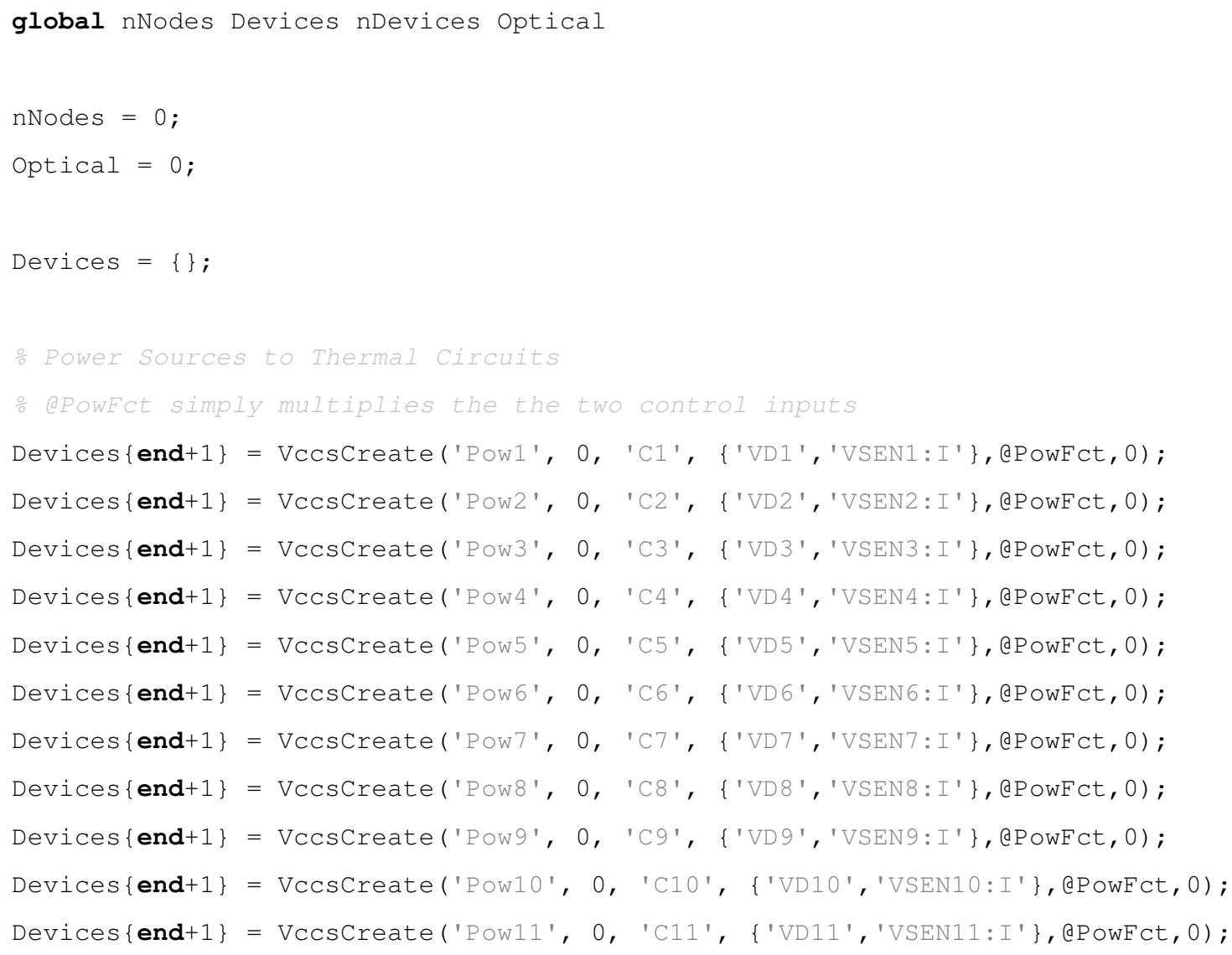




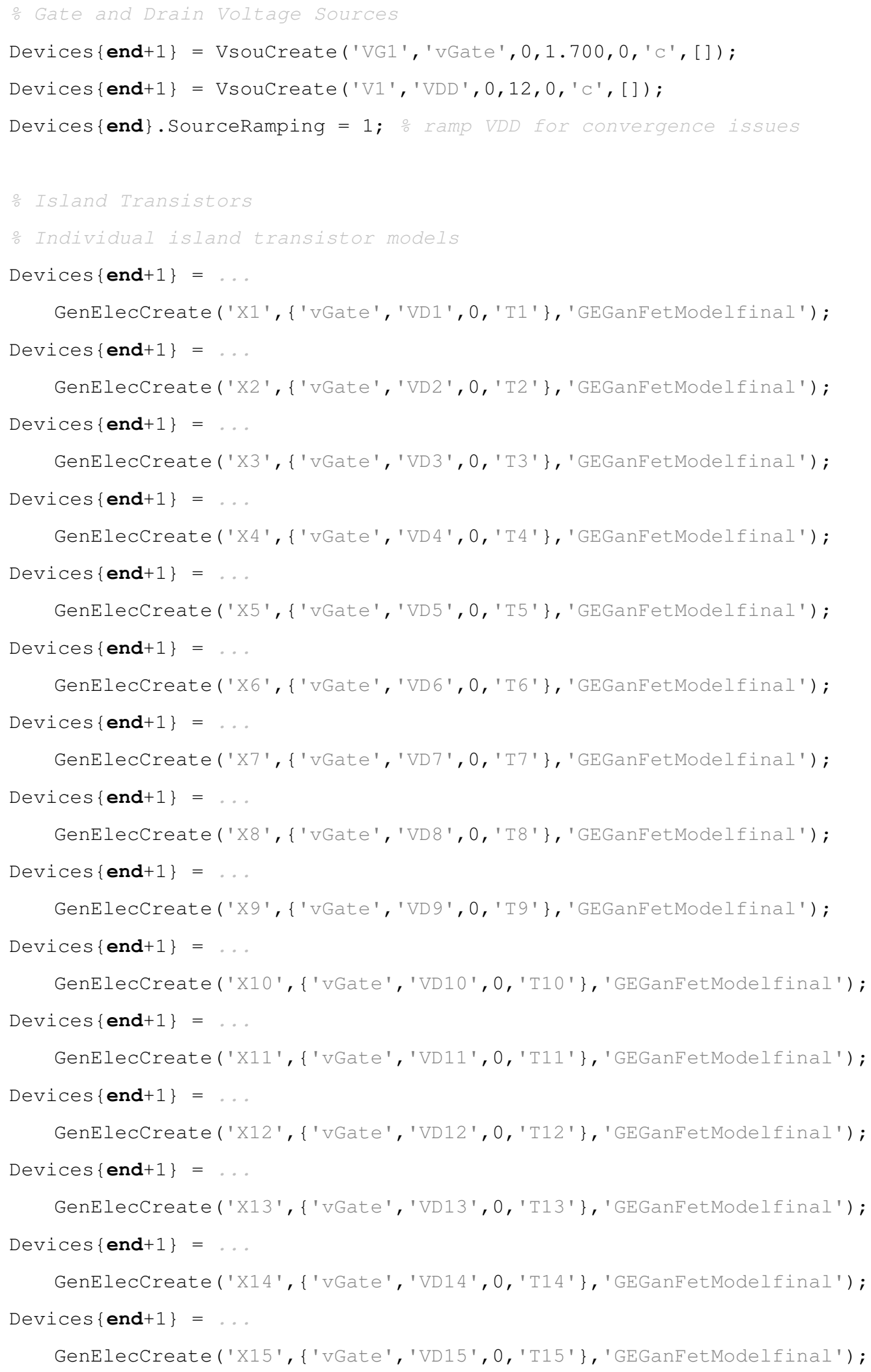




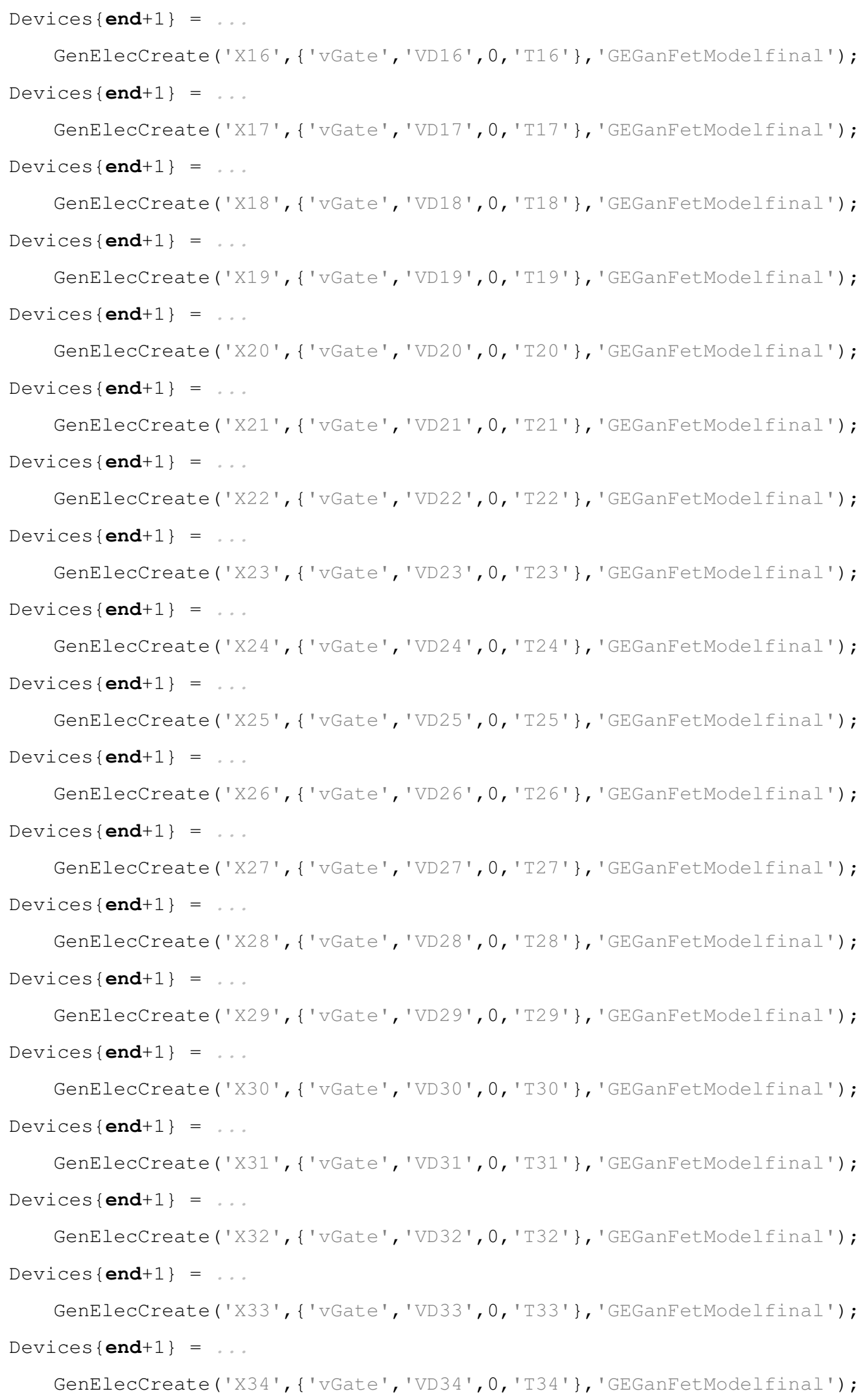




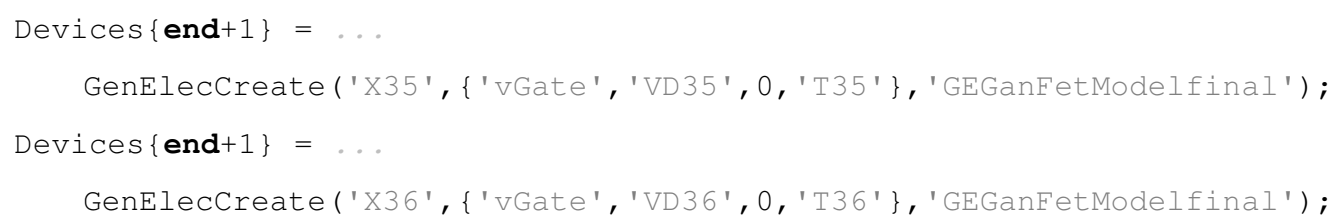




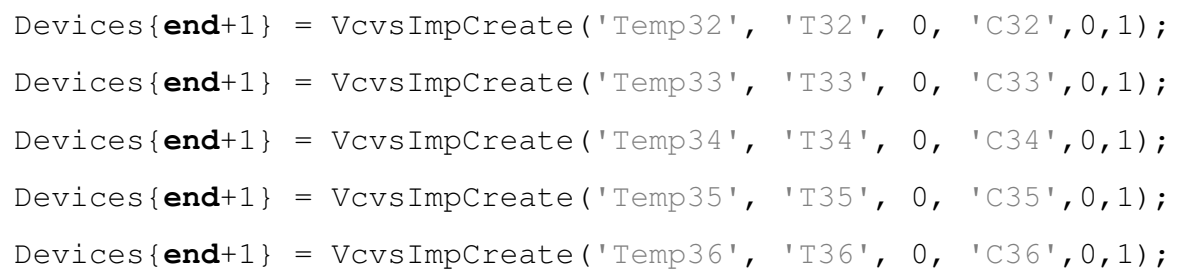




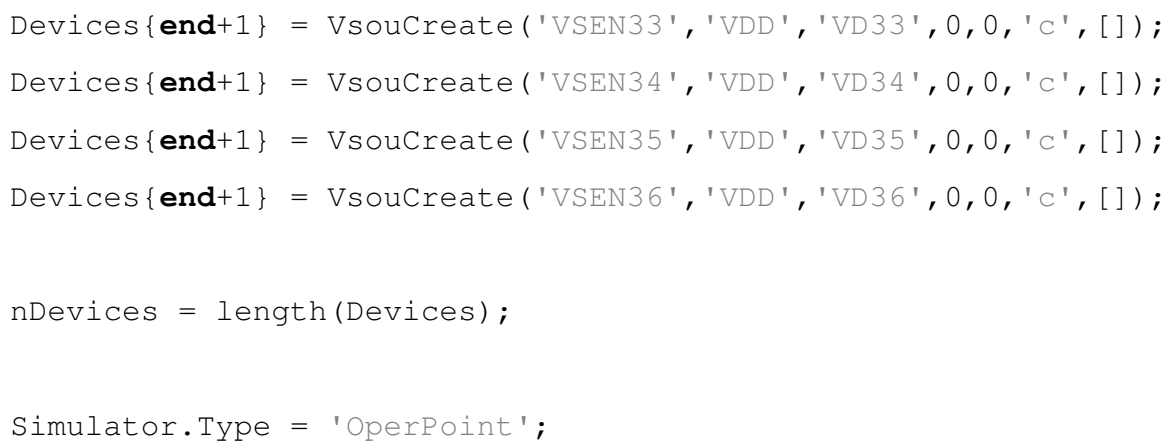




\section{B.2 OptiSPICE Electrical Simulation Input Code}

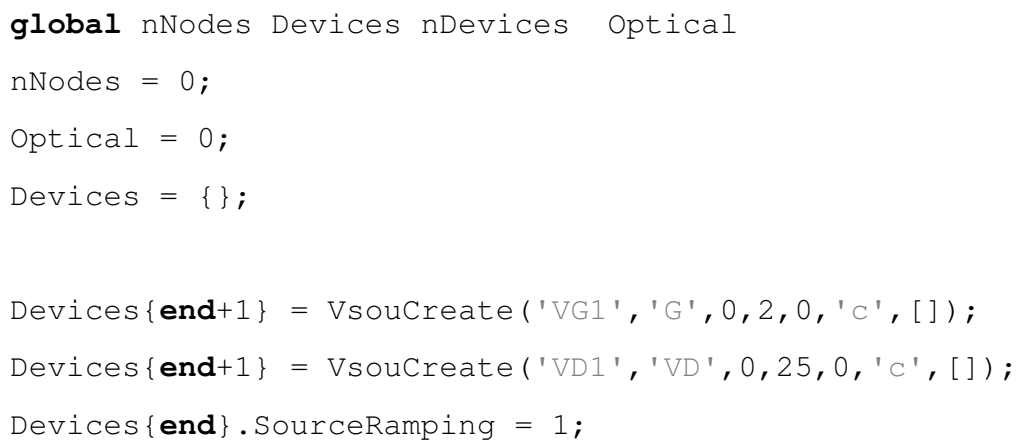




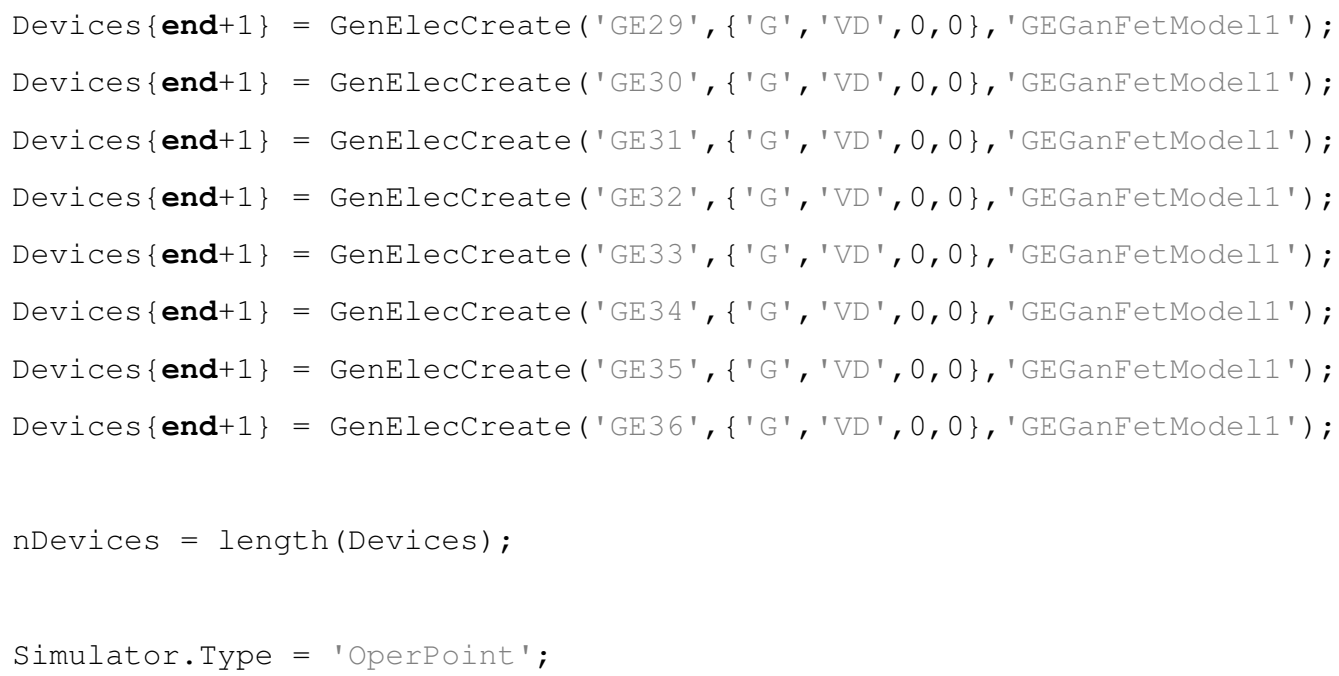




\section{Appendix $\mathrm{C}$}

\section{Simulation Output Files}

\section{C.1 Atar Thermal Simulation Comparison Output}

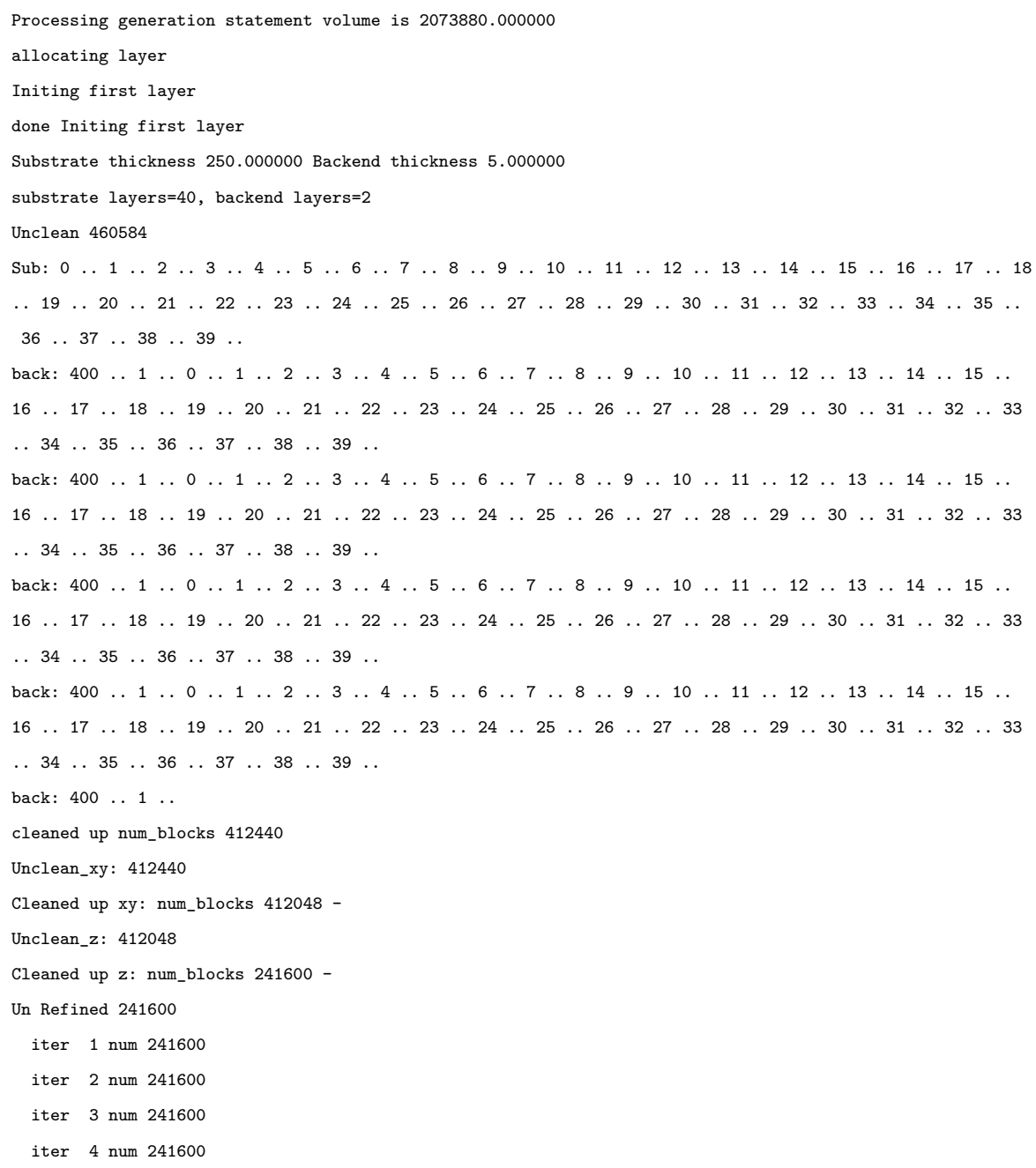




\author{
iter 5 num 241600 \\ iter 6 num 241600 \\ Refined 241600 \\ Number of blocks: 241600 \\ Number of blocks after padding: 241600 \\ Setting BC blocks \\ vnum 1024 inum 76680 mnum 0 lnum 0 fnum 0 pnum 0
}

EMnum 0 EM excitation 0

Using a total generation volume of 2052695.846558 initial vol 2073880.000000 total power of: 2.78 Using a total generation volume of 2052695.846558 initial vol 2073880.000000 total power of: 2.78 Using a total generation volume of 2052695.846558 initial vol 2073880.000000 total power of: 2.78 Using a total generation volume of 2052695.846558 initial vol 2073880.000000 total power of: 2.78 Using a total generation volume of 2052695.846558 initial vol 2073880.000000 total power of: 2.78 Using a total generation volume of 2052695.846558 initial vol 2073880.000000 total power of: 2.78 Power in model 16.68

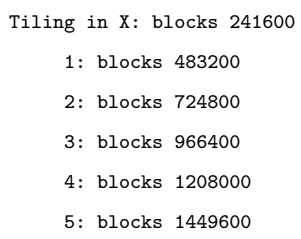




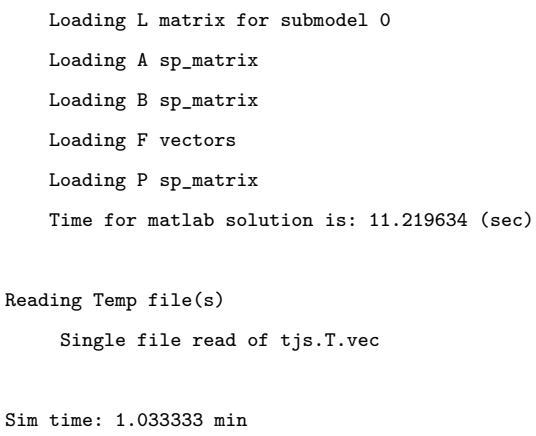




\section{C.2 OptiSPICE Electrothermal Simulation}

\section{Comparison Output}

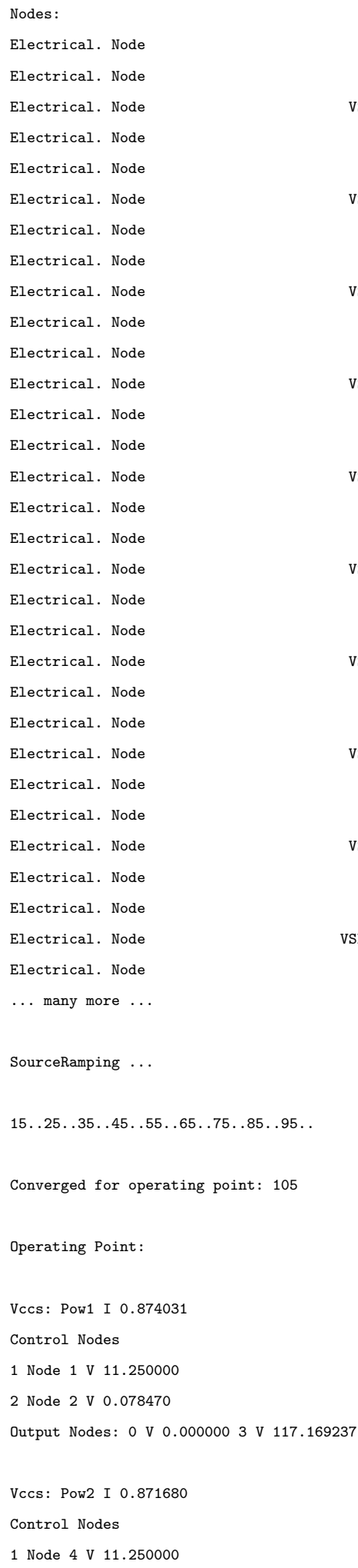


2 Node 5 V 0.078257

Output Nodes: 0 V 0.0000006 V 117.836325

Vccs: Pow3 I 0.871391

Control Nodes

1 Node 7 V 11.250000

2 Node 8 V 0.078231

Output Nodes: 0 V 0.0000009 V 117.918310

Vccs: Pow4 I 0.871402

Control Nodes

1 Node 10 V 11.250000

2 Node $11 \mathrm{~V} 0.078232$

Output Nodes: O V 0.00000012 V 117.915385

Vccs: Pow5 I 0.871722

Control Nodes

1 Node 13 V 11.250000

2 Node $14 \mathrm{~V} 0.078261$

Output Nodes: O V 0.00000015 V 117.824326

Vccs: Pow6 I 0.874157

Control Nodes

1 Node 16 V 11.250000

2 Node $17 \mathrm{~V} 0.078482$

Output Nodes: O V 0.00000018 V 117.133425

Vccs: Pow7 I 0.873323

Control Nodes

1 Node 19 V 11.250000

2 Node 20 V 0.078406

Output Nodes: O V 0.00000021 V 117.369937

Vccs: Pow8 I 0.870897

Control Nodes

1 Node 22 V 11.250000

2 Node 23 V 0.078186

Output Nodes: 0 V 0.00000024 V 118.058677

Vccs : Pow9 I 0.870589

Control Nodes

1 Node 25 V 11.250000

2 Node 26 V 0.078158

Output Nodes: O V 0.00000027 V 118.145989

Vccs: Pow10 I 0.870666

Control Nodes

1 Node 28 V 11.250000

2 Node 29 V 0.078165

Output Nodes: 0 V 0.00000030 V 118.124183

Vccs: Pow11 I 0.871496

Control Nodes

1 Node $31 \mathrm{~V} 11.250000$

2 Node 32 V 0.078240

Output Nodes: O V 0.00000033 V 117.888673 
Vccs: Pow12 I 0.874974

Control Nodes

1 Node 34 V 11.250000

2 Node $35 \mathrm{~V} 0.078556$

Output Nodes: O V 0.00000036 V 116.901421

Vccs : Pow13 I 0.873962

Control Nodes

1 Node 37 V 11.250000

2 Node 38 V 0.078464

Output Nodes: O V 0.00000039 V 117.188793

Vccs : Pow14 I 0.872217

Control Nodes

1 Node 40 V 11.250000

2 Node $41 \mathrm{~V} 0.078306$

Output Nodes: 0 V 0.00000042 V 117.683895

Vccs: Pow15 I 0.871204

Control Nodes

1 Node 43 V 11.250000

2 Node $44 \mathrm{~V} 0.078214$

Output Nodes: O V 0.00000045 V 117.971331

Vccs : Pow16 I 0.870929

Control Nodes

1 Node 46 V 11.250000

2 Node 47 V 0.078189

Output Nodes: O V 0.00000048 V 118.049382

Vccs : Pow17 I 0.873870

Control Nodes

1 Node $49 \mathrm{~V} 11.250000$

2 Node $50 \mathrm{~V} 0.078456$

Output Nodes: 0 V 0.00000051 V 117.214677

Vccs: Pow18 I 0.000000

Control Nodes

1 Node $52 \mathrm{~V} 11.250000$

2 Node 53 V 0.000000

Output Nodes: O V 0.00000054 V 112.657873

Vccs: Pow19 I 0.876295

Control Nodes

1 Node 55 V 11.250000

2 Node $56 \mathrm{~V} 0.078676$

Output Nodes: 0 V 0.00000057 V 116.526481

Vccs: Pow20 I 0.000000

Control Nodes

1 Node 58 V 11.250000

2 Node $59 \mathrm{~V} 0.000000$

Output Nodes: O V 0.00000060 V 113.694631

Vccs: Pow21 I 0.873574 


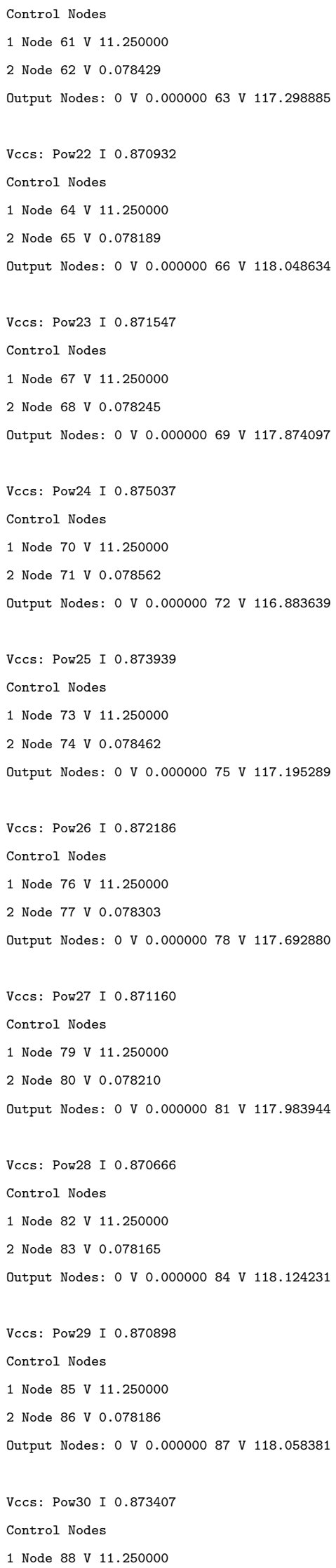


2 Node $89 \mathrm{~V} 0.078414$

Output Nodes: O V 0.00000090 V 117.346238

Vccs: Pow31 I 0.874066

Control Nodes

1 Node $91 \mathrm{~V} 11.250000$

2 Node 92 V 0.078474

Output Nodes: O V 0.00000093 V 117.159054

Vccs : Pow32 I 0.871726

Control Nodes

1 Node 94 V 11.250000

2 Node $95 \mathrm{~V} 0.078261$

Output Nodes: O V 0.00000096 V 117.823267

Vccs: Pow33 I 0.871415

Control Nodes

1 Node 97 V 11.250000

2 Node $98 \mathrm{~V} 0.078233$

Output Nodes: O V 0.00000099 V 117.911466

Vccs: Pow34 I 0.871389

Control Nodes

1 Node $100 \mathrm{~V} 11.250000$

2 Node $101 \mathrm{~V} 0.078231$

Output Nodes: O V 0.000000102 V 117.918989

Vccs: Pow35 I 0.871669

Control Nodes

1 Node 103 V 11.250000

2 Node $104 \mathrm{~V} 0.078256$

Output Nodes: O V 0.000000105 V 117.839592

Vccs: Pow36 I 0.874067

Control Nodes

1 Node 106 V 11.250000

2 Node 107 V 0.078474

Output Nodes: O V 0.000000108 V 117.158825

MatrixEl: M1

Port Vs: 117.169237

Port Vs: 117.836325

Port Vs: 117.918310

Port Vs: 117.915385

Port Vs: 117.824326

Port Vs: 117.133425

Port Vs: 117.369937

Port Vs: 118.058677

Port Vs: 118.145989

Port Vs: 118.124183

Port Vs: 117.888673

Port Vs: 116.901421

Port Vs: 117.188793

Port Vs: 117.683895

Port Vs: 117.971331 
Port Vs: 118.049382 Port Vs: 117.214677 Port Vs: 112.657873 Port Vs: 116.526481 Port Vs: 113.694631 Port Vs: 117.298885 Port Vs: 118.048634 Port Vs: 117.874097 Port Vs: 116.883639 Port Vs: 117.195289 Port Vs: 117.692880 Port Vs: 117.983944 Port Vs: 118.124231 Port Vs: 118.058381 Port Vs: 117.346238 Port Vs: 117.159054 Port Vs: 117.823267 Port Vs: 117.911466 Port Vs: 117.918989 Port Vs: 117.839592 Port Vs: 117.158825 Port Is : 0.882792

Port Is : 0.880394 Port Is : 0.880099 Port Is: 0.880110 Port Is: 0.880437 Port Is: 0.882920 Port Is : 0.882070 Port Is: 0.879595 Port Is: 0.879281 Port Is: 0.879359 Port Is : 0.880206 Port Is : 0.883754 Port Is : 0.882721 Port Is: 0.880942 Port Is: 0.879908 Port Is: 0.879628 Port Is : 0.882628 Port Is: -0.000000 Port Is: 0.885102 Port Is: -0.000000 Port Is: 0.882326 Port Is: 0.879631 Port Is: 0.880258 Port Is : 0.883818 Port Is: 0.882698 Port Is : 0.880909 Port Is : 0.879863 Port Is: 0.879359 Port Is : 0.879596 Port Is: 0.882155 Port Is : 0.882828 Port Is: 0.880441 Port Is : 0.880124 Port Is: 0.880097

Port Is : 0.880382 
Port Is : 0.882829

Node Vs (reduced): Max V: 118.656023

Vsou: VG1 Vin: 1.8281000 .000000 Iout: $-9.46685 \mathrm{e}-06$

Vsou: V1 Vin: 11.2500000 .000000 Iout: $-2.66304 \mathrm{e}+00$

Gen Elec: $\quad \mathrm{X} 1$

Node Voltages:

Vgin: $1.8281000000 \mathrm{e}+00$ Vdin: $1.1250000000 \mathrm{e}+01$ Vsin: $0.0000000000 \mathrm{e}+00$

VTO: $1.1716923734 \mathrm{e}+02$ Vd: $1.1189969432 \mathrm{e}+01$ Vd1: $1.1189969432 \mathrm{e}+01$

Vd2: $1.1189961585 \mathrm{e}+01$ Vd3: $1.1250000000 \mathrm{e}+01 \quad \mathrm{Vg}: 1.8280931169 \mathrm{e}+00$

Vg1: $1.8280931169 \mathrm{e}+00$ Vs: $3.3053385668 \mathrm{e}-03$ Vs1: $3.3053494011 \mathrm{e}-03$

Vs2: $3.3131855958 \mathrm{e}-03$ Vs3: $0.0000000000 \mathrm{e}+00$ VT1: $1.1875349461 \mathrm{e}+02$

$\mathrm{T}$ (Def.Cir) : $4.1690349461 \mathrm{e}+02$ del $\mathrm{T}: 1.1875349461 \mathrm{e}+02$

Resistors:

Rd: $\quad R=7.6501005587 \mathrm{e}-01 \quad \mathrm{~V}=-6.0035208503 \mathrm{e}-02 \quad \mathrm{I}=-7.8476365170 \mathrm{e}-02$

Rg: $\quad R=2.4720670391 \mathrm{e}+01 \quad \mathrm{~V}=6.8850292048 \mathrm{e}-06 \quad \mathrm{I}=2.7851304580 \mathrm{e}-07$

Rs: $\quad R=4.2122011173 \mathrm{e}-02 \quad V=3.3055940630 \mathrm{e}-03 \quad I=7.8476643704 \mathrm{e}-02$

Rd1: $\quad R=3.8078947368 \mathrm{e}-02 \quad V=1.5807088971 \mathrm{e}-10 \quad I=4.1511360118 \mathrm{e}-09$

Rs1: $\quad R=3.8078947368 \mathrm{e}-02 \quad \mathrm{~V}=-1.0834238664 \mathrm{e}-08 \quad \mathrm{I}=-2.8452043485 \mathrm{e}-07$

Rd2: $\quad R=1.0000000000 \mathrm{e}-04 \quad V=7.8476355956 \mathrm{e}-06 \quad I=7.8476355956 \mathrm{e}-02$

Rs2: $\quad R=1.0000000000 \mathrm{e}-04 \quad \mathrm{~V}=-7.8476355959 \mathrm{e}-06 \quad I=-7.8476355959 \mathrm{e}-02$

Rcgs: $\quad R=4.0000000000 \mathrm{e}+09 \quad \mathrm{~V}=1.8247875209 \mathrm{e}+00 \quad I=4.5619688023 \mathrm{e}-10$

Rcgd: $\quad R=4.0000000000 e+09 \quad V=-9.2493716765 e+00 \quad I=-2.3123429191 e-09$

Rcds: $\quad R=4.0000000000 \mathrm{e}+09 \quad \mathrm{~V}=1.1074159197 \mathrm{e}+01 \quad I=2.7685397994 \mathrm{e}-09$

Rth: $\quad R=1.7945004298 \mathrm{e}+00 \quad \mathrm{~V}=-1.5681061577 \mathrm{e}+00 \quad \mathrm{I}=-8.7383994546 \mathrm{e}-01$

Capacitors:

CGS: $\quad$ C $=9.7163608563 \mathrm{e}-12$

CGD: $\quad C=5.3033459255 \mathrm{e}-14$

CSD: $\quad C=3.9882044561 \mathrm{e}-12$

CSDM3: $\quad \mathrm{C}=8.5238095238 \mathrm{e}-13$

Cth: $\quad C=1.1187500000 \mathrm{e}-04$

Inductors:

$\mathrm{Ld}: \quad \mathrm{L}=4.0000000000 \mathrm{e}-11$

Ls: $\quad \mathrm{L}=4.0000000000 \mathrm{e}-11$

Lg: $\quad L=1.0000000000 \mathrm{e}-12$

Current Sources:

Ibsw: $\quad V=1.1074159197 \mathrm{e}+01 \quad I=7.8476120025 \mathrm{e}-02$

IbgsD1: $\quad V=1.8247875101 \mathrm{e}+00 \quad \mathrm{I}=2.8448518660 \mathrm{e}-07$

IbgdD1: $\quad \mathrm{V}=-9.2493716764 \mathrm{e}+00 \quad \mathrm{I}=-4.0992549523 \mathrm{e}-09$

IbgdD2: $\quad \mathrm{V}=-9.2493716764 \mathrm{e}+00 \quad \mathrm{I}=7.1632982177 \mathrm{e}-34$

IbdsD1: $\quad \mathrm{V}=1.1074159186 \mathrm{e}+01 \quad \mathrm{I}=4.7251734138 \mathrm{e}-15$

IbdsD2: $\quad \mathrm{V}=-1.1074159186 \mathrm{e}+01 \quad \mathrm{I}=1.1374731109 \mathrm{e}-11$

Ibtemp: $\quad V=1.1866624696 \mathrm{e}+02 \quad I=8.7407617298 \mathrm{e}-01$

Rth: $\quad V=-1.5681061577 \mathrm{e}+00 \quad I=-8.7383994546 \mathrm{e}-01$

NonLin Caps: 
Cgs: $\quad Q=1.0227907943 \mathrm{e}-10$
Cgd: $\quad Q=3.1576302001 \mathrm{e}-11$
Csd: $\quad Q=1.6693123395 \mathrm{e}-09$

Gen Elec:

$\mathrm{x} 2-\mathrm{x} 36 \ldots$ Cut for brevity

VCVS (imp): Control Nodes

1 Node 3 V 117.169237

2 Node 0 V 0.000000

Output Nodes: 149 V 117.169237 O V 0.000000

VCVS (imp): Control Nodes

1 Node 6 V 117.836325

2 Node 0 V 0.000000

Output Nodes: 162 V 117.8363250 V 0.000000

VCVS(imp): Control Nodes

1 Node 9 V 117.918310

2 Node 0 V 0.000000

Output Nodes: 175 V 117.918310 O V 0.000000

VCVS (imp): Control Nodes

1 Node 12 V 117.915385

2 Node 0 V 0.000000

Output Nodes: 188 V 117.915385 O V 0.000000

VCVS(imp): Control Nodes

1 Node 15 V 117.824326

2 Node 0 V 0.000000

Output Nodes: 201 V 117.8243260 V 0.000000

VCVS(imp): Control Nodes

1 Node 18 V 117.133425

2 Node 0 V 0.000000

Output Nodes: 214 V 117.1334250 V 0.000000

VCVS(imp): Control Nodes

1 Node $21 \mathrm{~V} 117.369937$

2 Node 0 V 0.000000

Output Nodes: 227 V 117.369937 O V 0.000000

VCVS (imp): Control Nodes

1 Node $24 \mathrm{~V} 118.058677$

2 Node $0 \mathrm{~V} 0.000000$

Output Nodes: 240 V 118.058677 O V 0.000000

VCVS (imp): Control Nodes

1 Node 27 V 118.145989

2 Node 0 V 0.000000

Output Nodes: 253 V 118.145989 O V 0.000000

VCVS(imp): Control Nodes

1 Node 30 V 118.124183

2 Node 0 V 0.000000 
Output Nodes: 266 V 118.124183 O V 0.000000

VCVS(imp): Control Nodes

1 Node 33 V 117.888673

2 Node 0 V 0.000000

Output Nodes: 279 V 117.8886730 V 0.000000

VCVS(imp): Control Nodes

1 Node 36 V 116.901421

2 Node 0 V 0.000000

Output Nodes: 292 V 116.9014210 V 0.000000

VCVS (imp): Control Nodes

1 Node 39 V 117.188793

2 Node 0 V 0.000000

Output Nodes: 305 V 117.188793 O V 0.000000

VCVS(imp): Control Nodes

1 Node 42 V 117.683895

2 Node 0 V 0.000000

Output Nodes: 318 V 117.683895 o V 0.000000

VCVS (imp): Control Nodes

1 Node 45 V 117.971331

2 Node 0 V 0.000000

Output Nodes: 331 V 117.9713310 V 0.000000

VCVS(imp): Control Nodes

1 Node 48 V 118.049382

2 Node 0 V 0.000000

Output Nodes: 344 V 118.049382 O V 0.000000

VCVS(imp): Control Nodes

1 Node $51 \mathrm{~V} 117.214677$

2 Node 0 V 0.000000

Output Nodes: 357 V 117.214677 O V 0.000000

VCVS(imp): Control Nodes

1 Node $54 \mathrm{~V} 112.657873$

2 Node 0 V 0.000000

Output Nodes: 371 V 112.657873 O V 0.000000

VCVS(imp): Control Nodes

1 Node 57 V 116.526481

2 Node $0 \mathrm{~V} 0.000000$

Output Nodes: 384 V 116.5264810 V 0.000000

VCVS(imp): Control Nodes

1 Node 60 V 113.694631

2 Node 0 V 0.000000

Output Nodes: 397 V 113.6946310 V 0.000000

$\operatorname{VCVS}(i m p):$ Control Nodes

1 Node 63 V 117.298885

2 Node 0 V 0.000000

Output Nodes: 410 V 117.298885 O V 0.000000 


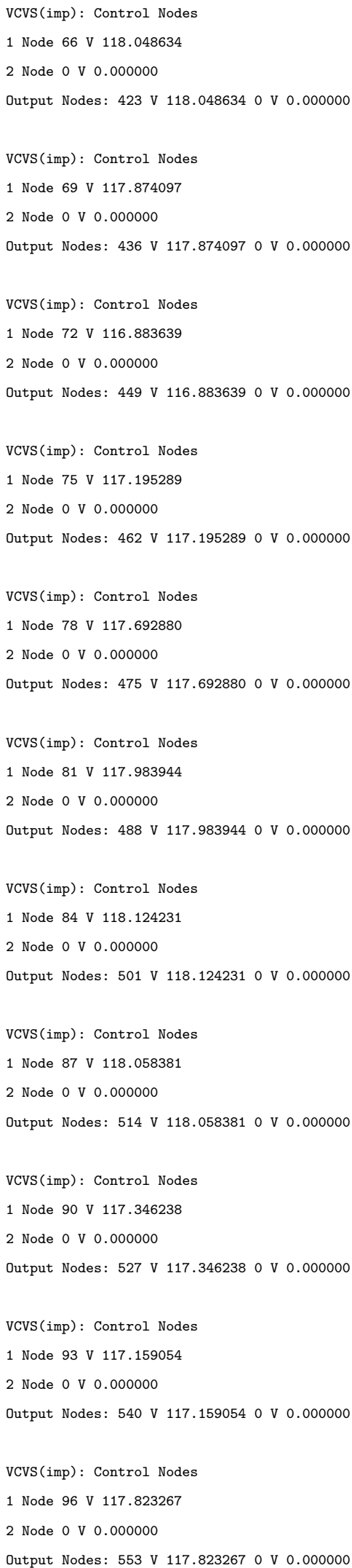




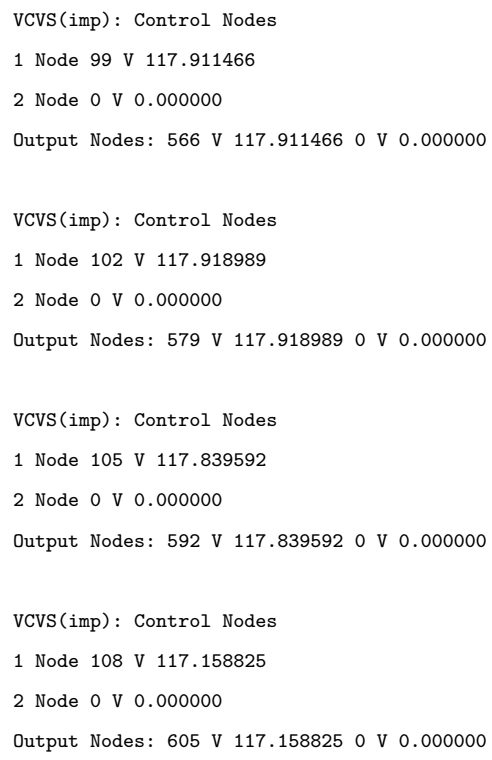

$7.84703 e-02$

$7.82571 \mathrm{e}-02$

$7.82309 \mathrm{e}-02$

$7.82319 \mathrm{e}-02$

$7.82610 \mathrm{e}-02$

$7.84817 \mathrm{e}-02$

$7.84062 \mathrm{e}-02$

$7.81861 \mathrm{e}-02$

$7.81582 \mathrm{e}-02$

$7.81652 \mathrm{e}-02$

$7.82404 \mathrm{e}-02$

7.85559e-02

$7.84641 \mathrm{e}-02$

$7.83059 \mathrm{e}-02$

$7.82140 \mathrm{e}-02$

$7.81891 \mathrm{e}-02$

7.84558e-02

$0.00000 \mathrm{e}+00$

$7.86757 \mathrm{e}-02$

$0.00000 \mathrm{e}+00$

$7.84289 \mathrm{e}-02$

$7.81893 e-02$

$7.82451 \mathrm{e}-02$

$7.85616 \mathrm{e}-02$

$7.84620 \mathrm{e}-02$

$7.83030 \mathrm{e}-02$

$7.82100 \mathrm{e}-02$

$7.81651 \mathrm{e}-02$

$7.81862 \mathrm{e}-02$

$7.84137 \mathrm{e}-02$

$7.84736 \mathrm{e}-02$

$7.82613 \mathrm{e}-02$

$7.82331 \mathrm{e}-02$

$7.82307 \mathrm{e}-02$

$7.82561 \mathrm{e}-02$

7.84736e-02 